\title{
A LANDING THEOREM FOR ENTIRE FUNCTIONS WITH BOUNDED POST-SINGULAR SETS
}

\author{
Anna Miriam Benini and @ ilasse Rempe
}

\begin{abstract}
The Douady-Hubbard landing theorem for periodic external rays is one of the cornerstones of the study of polynomial dynamics. It states that, for a complex polynomial $f$ with bounded postcritical set, every periodic external ray lands at a repelling or parabolic periodic point, and conversely every repelling or parabolic point is the landing point of at least one periodic external ray. We prove an analogue of this theorem for an entire function $f$ with bounded postsingular set. If $f$ has finite order of growth, then it is known that the escaping set $I(f)$ contains certain curves called periodic hairs; we show that every periodic hair lands at a repelling or parabolic periodic point, and conversely every repelling or parabolic periodic point is the landing point of at least one periodic hair. For a postsingularly bounded entire function $f$ of infinite order, such hairs may not exist. Therefore we introduce certain dynamically natural connected subsets of $I(f)$, called dreadlocks. We show that every periodic dreadlock lands at a repelling or parabolic periodic point, and conversely every repelling or parabolic periodic point is the landing point of at least one periodic dreadlock. More generally, we prove that every point of a hyperbolic set is the landing point of a dreadlock.
\end{abstract}

\section{Introduction}

Let $p: \mathbb{C} \rightarrow \mathbb{C}$ be a polynomial. The filled-in Julia set $K(p)$ consists of those points $z \in \mathbb{C}$ whose orbits remain bounded under repeated application of $p$. In their study of the dynamics of complex polynomials and the Mandelbrot set [DH85], Douady and Hubbard introduced the notion of external rays, which can be characterised as the gradient lines of the Green's function on the basin of attraction of infinity, $\mathbb{C} \backslash K(p)$. Periodic (and pre-periodic) rays are of particular importance, due to the following result.

Anna Miriam Benini: was supported by the European Union's Horizon 2020 research and innovation programme under the Marie Skłodowska-Curie Grant Agreement No. 703269 COTRADY and by the SIR Grant NEWHOLITE No. RBSI14CFME. The second author was partially supported by a Philip Leverhulme Prize

Keywords and phrases: Transcendental entire function, Transcendental dynamics, Accessibility, Combinatorics, External ray, Hair, Dreadlock

Mathematics Subject Classification: Primary 37F20; Secondary 30D05, 37F10, 37F12 
Douady-Hubbard landing theorem. Let $p$ be a polynomial whose post-critical set

$$
\mathcal{P}(p):=\bigcup_{c: p^{\prime}(c)=0}\left\{p^{n}(c): n \geq 1\right\}
$$

is bounded. (Equivalently, assume that $K(p)$ is connected.)

Then every periodic ray of $p$ lands at a repelling or parabolic periodic point, and conversely every repelling or parabolic periodic point of $p$ is the landing point of at least one and at most finitely many periodic external rays.

The first half of this theorem, concerning the landing of periodic rays, can be found in [DH85, Exposé VIII.II, Proposition 2]. The second half, which is more difficult, is due to Douady; the first published proofs are in [EL89, Hub93]. Ever since, the Douady-Hubbard theorem has been a cornerstone of the study of polynomial dynamics. In particular, it forms the basis of the "puzzle techniques" that were pioneered by Yoccoz, Branner and Hubbard, and continue to lead to fundamental new results; see [Hub93, RY08, ALS11].

In the study of rational functions and transcendental entire functions, there is no immediate analogue of the basin of infinity, and this is one of the reasons that the study of these classes has presented greater challenges than that of polynomials. Nonetheless, in both settings analogues of the above-mentioned puzzle techniques have been employed to certain classes of functions with considerable success. We refer to [Roe08, Ben15] for two examples.

Our goal is to extend the Douady-Hubbard landing theorem to the case of a transcendental entire function $f$. In this setting, the role that critical values play in polynomial dynamics is taken by the larger set $S(f)$ of singular values of $f$. These are those points not having a neighbourhood in which all branches of $f^{-1}$ are defined and holomorphic. Analogously to (1.1), the postsingular set of $f$ is defined as

$$
\mathcal{P}(f):=\overline{\bigcup_{s \in S(f)}\left\{f^{n}(s): n \geq 0\right\}} .
$$

For transcendental maps, $\infty$ is an essential singularity, rather than a superattracting fixed point. Hence the definition of external rays for polynomials as gradient lines of a Green's function has no natural analogue. Nonetheless, it has long been known that the escaping set

$$
I(f):=\left\{z \in \mathbb{C}: f^{n}(z) \rightarrow \infty\right\}
$$

often contains curves to infinity; indeed, in some cases this was already noticed by Fatou [Fat26]. It was the work of Devaney and his collaborators (see e.g. [DK84, DT86]) that really began the study of these hairs or dynamic rays in the 1980s, particularly for functions in the exponential family,

$$
f_{a}: z \mapsto e^{z}+a .
$$


Devaney, Goldberg and Hubbard were probably the first to suggest that such hairs could serve as analogues of external rays of polynomials; compare [DGH86, BDH+99]. Subsequently, Schleicher and Zimmer [SZ03a] and Schleicher and Rottenfußer [RS08b] proved that, for the families of exponential maps (1.3) and of cosine maps $z \mapsto$ $a e^{z}+b e^{-z}$, respectively, the entire escaping set $I(f)$ consists of hairs.

On the other hand, in [RRRS11] it is shown that there is a transcendental entire function $f$ for which $I(f)$ contains no arcs. Hence there are no curves in $I(f)$, of any kind, landing at any of the repelling periodic points of $f$. (Recall that repelling periodic points are dense in the Julia set of any transcendental entire function.) Furthermore, the postsingular set $\mathcal{P}(f)$ of this function is bounded. Indeed, $S(f)$ is a compact subset of the immediate basin of attraction of a single attracting fixed point.

Dreadlocks. In view of the preceding example, we develop a novel approach to the landing problem that removes the focus on hairs altogether, by connecting repelling periodic points to infinity using more general sets of escaping points.

More precisely, we introduce a notion of dreadlocks for postsingularly bounded entire functions. These are certain unbounded connected sets of escaping points generalising the concept of hairs. (See Section 4 for formal definitions.) The set of dreadlocks has a natural combinatorial structure, and in tame cases, all dreadlocks are in fact hairs. In general, however, dreadlocks can be topologically much more complicated. Indeed, it follows from [Rem16] that the closure of a dreadlock may be a hereditrarily indecomposable continuum. ${ }^{1}$

With this terminology, we are able to prove the following generalisation of the Douady-Hubbard landing theorem for postsingularly bounded entire functions: Every periodic dreadlock lands, and every repelling or parabolic periodic point is the landing point of at least one and at most finitely many periodic dreadlocks (Theorem 8.1). In particular, without requiring the definitions of Section 4, we can state the following result.

Theorem 1.1 (Landing at periodic points). Let $f$ be a transcendental entire function such that $\mathcal{P}(f)$ is bounded, and let $\zeta$ be a repelling or parabolic periodic point. Then there is a connected and unbounded set $A \subset I(f)$ and a period $p$ with the following properties.

(a) $\bar{A}=A \cup\{\zeta\}$ and $\bar{A}$ does not separate the plane;

(b) $f^{p}(A)=A$ and $f^{j}(A) \cap A=\emptyset$ for $1 \leq j<p$;

(c) for every $\varepsilon>0, f^{n}$ tends to $\infty$ uniformly on $\{z \in A:|z-\zeta| \geq \varepsilon\}$.

If $\tilde{\zeta} \neq \zeta$ is a different repelling or parabolic periodic point and $\tilde{A}$ is a set as above for $\tilde{\zeta}$ then $A \cap \tilde{A}=\emptyset$.

1 More precisely, [Rem16] shows that a Julia continuum of a disjoint-type entire function may have this property, Every such Julia continuum is the closure of a dreadlock in our sense; compare Lemma 4.14 and Remark 4.15. 
We emphasise that using dreadlocks, rather than restricting to cases where hairs exist (see Theorem 1.4 below), is crucial if one wishes to obtain results for general classes of functions. Indeed, Pfrang [Pfr19] uses our results to construct (homotopy) Hubbard trees for all postsingularly finite entire functions. This is a natural result whose hypothesis and conclusion make no mention of hairs; its proof in this form is made possible by the use of dreadlocks; compare the discussion at the end of the final section of [PRS18]. Similarly, work of Fagella and the first author [BF15, BF17, BF20], was formulated only for functions with hairs, but contains a number of results whose conclusion makes sense without this assumption. For example, the conclusion of the main theorem of [BF17] states that every non-repelling cycle has a singular orbit that is associated to it in a certain explicit manner. These results should now extend to all postsingularly bounded entire functions, by replacing the role of hairs in the proofs by our "dreadlocks". In addition, the key technique of fundamental tails that we use to control dreadlocks (see Section 3) has already found further applications, for instance in the study of inner functions arising in transcendental dynamics [EFJS19], and in a new version of the Fatou-Shishikura inequality [BF20].

Moreover, our results offer the possibility of developing puzzle-type arguments for all postsingularly bounded entire functions, and of using the powerful techniques of symbolic dynamics to study the behaviour of non-escaping points. As mentioned above, this is the reason why the structure of polynomial Julia sets is so well understood. Theorem 1.1 opens up large classes of entire transcendental functions to the same type of analysis.

Existence and landing of periodic hairs. In many interesting cases, periodic dreadlocks are in fact be periodic hairs. That is, the connected set $A$ in Theorem 1.1 is an arc connecting $\zeta$ to $\infty$. In particular, this holds for functions satisyfing the following property, which states that the escaping set consists entirely of hairs.

Definition 1.2 (Criniferous functions). We say that an entire function $f$ is criniferous $^{2}$ if the following holds for every $z \in I(f)$ : For all sufficiently large $n$ there is an arc $\gamma_{n}$ connecting $f^{n}(z)$ to $\infty$, in such a way that $f$ maps $\gamma_{n}$ injectively onto $\gamma_{n+1}$, and such that $\min _{z \in \gamma_{n}}|z| \rightarrow \infty$ as $n \rightarrow \infty$.

The counterexample from [RRRS11] mentioned above shows that entire functions, even those with bounded postsingular sets, need not be criniferous. However, the same article also establishes criniferousness for a large and natural class of functions, as follows. The Eremenko-Lyubich class $\mathcal{B}$ consists of those transcendental entire functions for which $S(f)$ is bounded, and hence compact. (If $\mathcal{P}(f)$ is bounded, then $f \in \mathcal{B}$ by definition.) It is proved in [RRRS11] that $f$ is criniferous whenever $f \in \mathcal{B}$ and $f$ has finite order of growth, i.e.,

$$
\log \log |f(z)|=O(\log |z|) .
$$

2 "Criniferous" means "having hair" or "hairy", from Latin crinis (hair) + ferre (to bear). 
Furthermore, any finite composition of functions with these properties is also criniferous.

To discuss periodic hairs, let us use the following definition from [Rem08].

DEFINITION 1.3 (Periodic hairs). An invariant hair of a transcendental entire function $f$ is a continuous and injective curve $\gamma: \mathbb{R} \rightarrow I(f)$ such that $f(\gamma(t))=\gamma(t+1)$ for all $t$ and $\lim _{t \rightarrow+\infty}|\gamma(t)|=\infty$. A periodic hair is a curve that is an invariant hair for some iterate $f^{n}$ of $f$.

Such a hair lands if the limit $z_{0}=\lim _{t \rightarrow-\infty} \gamma(t)$ exists; this limit is called the landing point (sometimes also endpoint) of the hair $\gamma$.

With this terminology, Theorem 1.1 takes the following form for criniferous functions.

Theorem 1.4. "Landing theorem for periodic hairs." Let $f$ be a transcendental entire function such that the postsingular set $\mathcal{P}(f)$ is bounded. Then every periodic hair of $f$ lands at a repelling or parabolic periodic point. If, in addition, $f$ is criniferous, then conversely every repelling or parabolic periodic point of $p$ is the landing point of at least one and at most finitely many periodic hairs.

The first part of the theorem, concerning landing behaviour of periodic rays, is not new. It was proved for exponential maps in [SZ03b], and later in full generality by the second author [Rem08, Corollary B.4]; see also [Den14]. The proof uses similar ideas as in the polynomial case, namely expansion properties for the hyperbolic metric, although there are also some additional ingredients.

On the other hand, the usual proofs for accessibility of repelling and parabolic periodic points in the polynomial case [EL89, Hub93, Prz94] strongly rely on the presence of the open basin of attraction of infinity, and thus break down completely in the transcendental setting. Nonetheless, there has been some previous work in this direction. Under the additional dynamical assumption that $f$ is geometrically finite, the theorem was proved by Mihaljević-Brandt [Mih10]. Furthermore, the first author and Lyubich [BL14] proved Theorem 1.4 when $f$ belongs to the exponential family (1.3).

For exponential maps, boundedness of the postsingular set is a strong dynamical condition (though weaker than geometrical finiteness), as it implies non-recurrence of the singular value $a$. However, the non-recurrence property is not used in any essential way in [BL14], and the ideas used there form one of the ingredients in our proofs of Theorems 1.1 and 1.4 .

During the preparation of this manuscript, Dierk Schleicher informed us that he has an alternative approach to Theorem 1.4, using ideas from [SZ03b].

Hyperbolic sets. As in [BL14], our techniques apply not only to repelling (and parabolic) periodic points, but also to hyperbolic sets; see [Prz94] for the corresponding result for polynomials. Recall that a compact, forward-invariant set $K \subset \mathbb{C}$ is called hyperbolic if for some $k \in \mathbb{N}$ and $\eta>1$ we have $\left|\left(f^{k}\right)^{\prime}(z)\right|>\eta$ for all $z \in K$. 
If $\mathcal{P}(f)$ is bounded and $K$ is such a hyperbolic set, then we prove that every point of $K$ is "accessible" from the escaping set, via a dreadlock (see Theorem 8.2). Again, we can state the following result without requiring the terminology of dreadlocks.

Theorem 1.5. "Landing at hyperbolic sets." Let $f$ be a transcendental entire function such that $\mathcal{P}(f)$ is bounded, and let $K$ be a hyperbolic set of $f$. Then there is a collection $\mathcal{A}$ of pairwise disjoint, connected and unbounded sets $A \subset I(f)$ with the following properties.

(a) For every $A \in \mathcal{A}$, there is $z_{0}(A) \in K$ such that $\bar{A}=A \cup\left\{z_{0}(A)\right\}$, and $\bar{A}$ does not separate the plane;

(b) the function $\mathcal{A} \rightarrow K ; A \mapsto z_{0}(A)$ is surjective;

(c) $f(A) \in \mathcal{A}$ for all $A \in \mathcal{A}$;

(d) for every $\varepsilon>0$, $f^{n}$ tends to $\infty$ uniformly on $\{z \in \bigcup \mathcal{A}$ : $\operatorname{dist}(z, K) \geq \varepsilon\}$;

(e) if $z_{0}(A)$ is periodic of period $p$, then $f^{k p}(A)=A$ for some $k \geq 1$.

If $f$ is criniferous, then every $A \in \mathcal{A}$ is an arc connecting $z_{0}(A) \in K$ to $\infty$.

This generalisation is of particular relevance in the case where $\mathcal{P}(f)$ itself is a hyperbolic set, which is often the case for non-recurrent entire functions (see [RvS11]). Hence, in this case, each singular value can itself be connected to infinity by a dreadlock, which in turn allows one to study the Julia set via symbolic dynamics rather closely. For example, in [Ben15], the existence of a ray landing at the omitted value is exploited to prove strong rigidity properties of non-recurrent parameters in the exponential family, extending previous work [Ben11] in the postsingularly finite case.

To conclude the introduction, we remark on the case where the postsingular set $\mathcal{P}(f)$ is unbounded. If $f$ is a polynomial, then every unbounded orbit escapes to infinity. For polynomials with escaping singular orbits, the Douady-Hubbard landing theorem no longer holds. Indeed, it is possible that a repelling periodic point is the landing point of uncountably many external rays, none of which are periodic. Compare [LP96].

For transcendental entire functions, it is possible for singular orbits to be unbounded without converging to infinity. It is conceivable that, for $f \in \mathcal{B}$ with all singular orbits nonescaping, a version of the landing theorem holds. However, even for exponential maps this is not known (see [Rem06a] for a partial result), and it appears that significant further new ideas would be required to approach it. See Section 14 for further discussion.

Structure of the paper. Section 2 gives an overview of expansivity properties for functions in class $\mathcal{B}$ without the assumption of bounded postsingular set. It also defines the concept of external addresses, and gives sufficient conditions on such addresses to be realised by certain unbounded connected sets of points. Several of the ideas used in this section are already implicitly or explicitly contained in the literature, e.g. in [EL92, Rem07a, Rem08, Rem09], but are combined here in a novel, systematic and unified manner. 
From Section 3 onward, we restrict to functions with bounded postsingular sets, beginning by discussing hyperbolic expansion estimates for such maps, and introducing the important combinatorial notion of fundamental tails. With these preparations, Section 4 introduces dreadlocks for a function with bounded postsingular set, studies their main topological and combinatorial properties, and also shows that the escaping set consists of dreadlocks. The ideas in this section have their roots in [Rem07a]. In particular, we recover the main result of that paper; see Corollary 4.11. In Section 5, we discuss the relation between dreadlocks and hairs.

Section 6 introduces accumulation sets of dreadlocks at bounded addresses, and gives different characterisations of when a dreadlock lands. This section contains a crucial innovation, which is central to the proofs of our main theorems: Rather than having to contend with the potentially complicated topological structure of dreadlocks, we can instead study their landing properties by considering a certain chain of open simply-connected sets. In Section 7, we establish that such a landing dreadlock cannot separate the plane. We are then ready to state our main theorems concerning dreadlocks in Section 8, and to derive Theorems 1.1 and 1.4 from these.

The three following sections are dedicated to proving the main theorems of this paper. Section 9 establishes the landing of periodic dreadlocks, in Section 10 we show accessibility of hyperbolic sets and repelling periodic orbits, and finally Section 11 is dedicated to the proof of accessibility of parabolic points.

We remark that one can take an alternative, less natural but more direct, approach to establishing our theorems, bypassing most of the material in Sections 2 and 4-6. Readers interested in such a short-cut are referred to Remark 8.4.

To round off the paper, Section 12 discusses bounds on the number of rays landing together at a given point in a hyperbolic set. We also include two appendices. The first, Section 13, gives some details concerning the cyclic order at infinity of unbounded connected sets, which are used in some of our arguments. The second, Section 14, discusses open questions about landing theorems for entire functions with unbounded postsingular sets.

Notation and preliminaries. We write $\mathbb{C}$ for the complex plane and $\hat{\mathbb{C}}$ for the Riemann sphere. We denote the closure in $\mathbb{C}$ of a set $A \subset \mathbb{C}$ by $\bar{A}$, and occasionally $\operatorname{cl}(A)$. The closure of $A$ in $\hat{\mathbb{C}}$ is denoted by $\hat{A}$.

The Euclidean disk of radius $R$ around a point $z$ is denoted by $D_{R}(z)$; the unit disk is $\mathbb{D}:=D_{1}(0)$. If $D$ is any Euclidean disc, we also write $r(D)$ for the radius of $D$.

We denote Euclidean distance and diameter by dist and diam, respectively. If $U \subset \mathbb{C}$ is an open set omitting more than two points, then we denote hyperbolic distance on $U$ by $\operatorname{dist}_{U}$, and similarly $\operatorname{diam}_{U}$ for hyperbolic diameter. We also denote the density of the hyperbolic metric of $U$ at a point $z$ by $\rho_{U}(z)$. That is, the length element of the hyperbolic metric is given by $\rho_{U}(z)|\mathrm{d} z|$. 


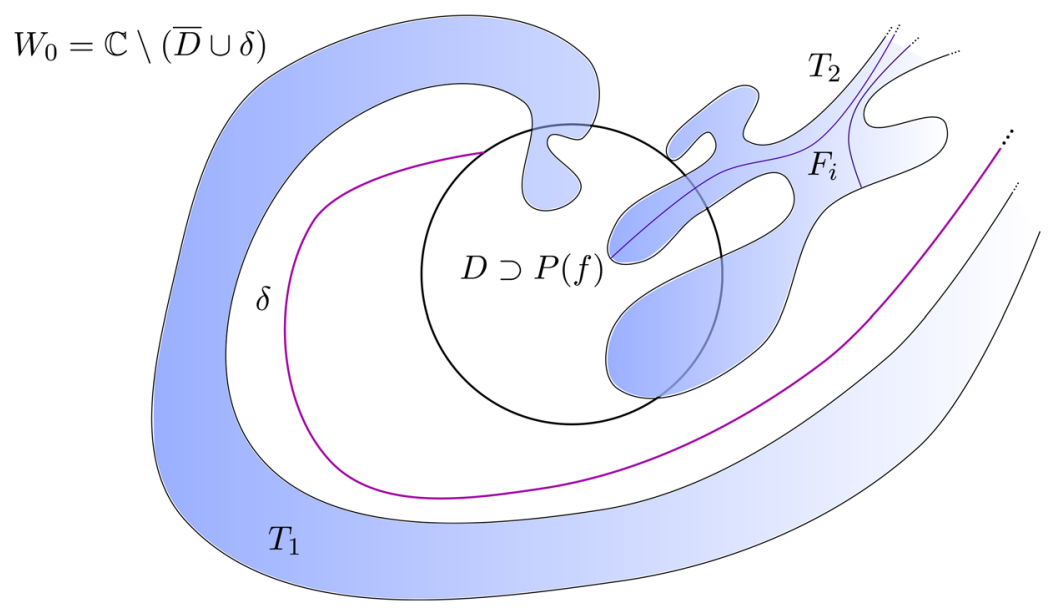

Figure 1: The dynamical plane for a function with two tracts $T_{1}$ and $T_{2}$. One of the fundamental domains $F_{i}$ obtained by taking preimages of $\delta$ is shown inside $T_{2}$.

\section{Unbounded Sets of Escaping Points}

In this section, we briefly review basic properties of the dynamics of a function $f \in \mathcal{B}$, and review the definition of external addresses for such maps. Then we state a theorem (Theorem 2.5) about the existence of unbounded connected sets for these addresses, and devote the rest of the section to the proof thereof. These sets will provide the basis of the "dreadlocks" that are introduced (for postsingularly bounded entire functions) in Section 4.

Throughout this section, fix a function $f \in \mathcal{B}$. Recall that this implies that $S(f)$ is bounded. For now, we do not assume that $\mathcal{P}(f)$ is also bounded. Let us begin by reviewing the method of partitioning the locus where $f$ is large into (topological) half-strips known as fundamental domains. (Compare e.g. [Rem08, Section 2] or [Rot05, Section 2].) For this construction, we fix a Euclidean disk $D$ around the origin containing $S(f)$. The connected components of $f^{-1}(\mathbb{C} \backslash \bar{D})$ are called the tracts of $f$. If $T$ is a tract, then $f: T \rightarrow \mathbb{C} \backslash \bar{D}$ is a universal covering map; in particular, $T$ is unbounded and simply connected. In fact (applying the same argument to a slightly smaller disc than $D), T$ is a Jordan domain in $\hat{\mathbb{C}}$ whose boundary passes through infinity, and $f$ is a universal covering $f: \bar{T} \rightarrow \mathbb{C} \backslash D$ on the closure of $T$ (in the complex plane $\mathbb{C}$ ).

We may assume in the following that $D \cap f(D) \neq \emptyset$, e.g. by ensuring that, $f(0) \in D$. Then it is easy to see that there is an $\operatorname{arc} \delta$ connecting a point of $\partial D$ to infinity in the complement of the closure of the tracts. We define

$$
W_{0}:=\mathbb{C} \backslash(\bar{D} \cup \delta)
$$

The connected components of $f^{-1}\left(W_{0}\right)$ are called the fundamental domains of $f$; see Figure 1. 
We remark that there are only finitely many fundamental domains that intersect a given compact set, due to the following simple fact.

Lemma 2.1 (Preimage components intersecting a compact set). Let $f: X \rightarrow Y$ be a holomorphic map between Riemann surfaces $X$ and $Y$. Furthermore, let $U \subset Y$ be a domain whose boundary (in $Y$ ) is locally connected; i.e. every point of the boundary of $U$ in $Y$ has arbitrarily small connected relative neighbourhoods in $Y$.

Then for any compact set $K \subset X$, only finitely many connected components of $f^{-1}(U)$ intersect $K$.

REMARK. The condition that $\partial U$ is locally connected is necessary: Let $X=Y=\mathbb{C}$, $f=\exp$, and let $U$ be a simply-connected domain in the punctured unit disc that spirals in towards the unit circle. (I.e., any branch of the argument on $U$ tends to infinity as $|z| \rightarrow 1$ in $U$.) Then infinitely many components of $f^{-1}(U)$ intersect the closed unit disc.

Proof. We begin by reformulating the hypothesis that $\partial U$ is locally connected, as follows.

Claim. Let $\zeta \in \bar{U}$, and let $\Delta$ be a neighbourhood of $\zeta$ in $Y$. Then there is a finite collection of connected open sets $W_{1}, \ldots, W_{n} \subset V \cap U$ such that $\{\zeta\} \cup W_{1} \cup \cdots \cup W_{n}$ is a neighbourhood of $\zeta$ in $\{\zeta\} \cup U$.

Proof. Shrinking $\Delta$ if necessary, we may assume that $\bar{\Delta}$ is a closed topological disc, and that $U \not \subset \Delta$. Consider the compact set $Q:=\partial \Delta \cup(\Delta \backslash U)$. Since $U$ is connected but not contained in $\Delta$, the boundary of each connected component of $U \cap \Delta=\Delta \backslash Q$ intersects $\partial \Delta$. Recall that $\partial U$ is locally connected; it follows readily that $Q$ is also. Hence $U \cap \Delta$ has at most finitely many connected components of diameter greater than, say, $\delta:=\operatorname{dist}(\zeta, \partial \Delta) / 2$; see [Why42, Theorem 4.4 in Chapter VI]. (Here dist refers to distance with respect to some metric on the topological disc $\bar{\Delta}$.) Therefore only finitely many connected components of $W_{1}, \ldots, W_{n}$ of $U \cap \Delta$ intersect the disc of radius $\delta$ around $\zeta$, as claimed.

Let $z \in f^{-1}(\bar{U})$, and $\zeta:=f(z)$. Then $z$ has a neighbourhood $V_{1}$ that is topologically mapped as by $z \mapsto z^{d}$, where $d$ is the local degree of $f$ at $z$. Take $W_{1}, \ldots, W_{n}$ as in the claim, for $\Delta=f\left(V_{1}\right)$. If $V_{z} \subset V_{1}$ is a sufficiently small disc around $z$, then any point in $f^{-1}(U) \cap V_{z}$ maps into some $W_{j}$. As $f^{-1}\left(W_{j}\right)$ has $d$ connected components in $V_{1}$, it follows that $V_{z}$ intersects at most $d n$ connected components of $f^{-1}(U)$.

So the compact set $K \cap f^{-1}(\bar{U})$ has an open cover by sets $V_{z}$, each of which intersects only finitely many connected components of $f^{-1}(U)$. The claim follows by taking a finite subcover.

It follows that there are only finitely many fundamental domains $F$ whose closure intersects the disc $\bar{D}$. When this does not occur for any $F$, the function $f$ is dynamically particularly simple; more precisely, it is of disjoint type (hyperbolic with connected Fatou set). For a detailed study of the topological dynamics of such 
functions, see [Rem16]. (Compare also the discussion of disjoint-type addresses in Remark 4.15). In the following, given a fundamental domain $F$ we denote by $\stackrel{\infty}{F}$ the unbounded connected component of $F \backslash \bar{D}$.

Expansion properties and relative cylindrical distance. It is known that functions in $\mathcal{B}$ are strongly expanding near infinity. More precisely, if $f \in \mathcal{B}$, then the cylindrical derivative of $f$ is large whenever $f(z)$ is large [EL92, Lemma 1]. That is,

$$
\|\mathrm{D} f(z)\|_{\mathrm{cyl}}:=\left|f^{\prime}(z) \cdot \frac{z}{f(z)}\right| \rightarrow \infty \quad \text { as } \quad|f(z)| \rightarrow \infty .
$$

In view of (2.2), we may assume that the radius $r(D)$ is chosen sufficiently large to ensure that

$$
\|\mathrm{D} f(z)\|_{\mathrm{cyl}} \geq 2
$$

whenever $f(z) \notin D$. In particular, $f(0) \in D$. These assumptions will remain in place for the remainder of the paper.

A number of results in the literature are phrased not for the function $f$ directly, but in terms of a logarithmic transform $\mathcal{L}$ of $f$. (See e.g. [EL92] or [Rem07a].) Such a transform can be obtained using the change of variable $z=\rho \cdot \exp (\zeta)$, where $\rho=r(D)$ is the radius of $D$. I.e., there is a $2 \pi i$-periodic function $\mathcal{L}$ defined by

$$
\rho \cdot \exp (\mathcal{L}(\zeta))=f(\rho \cdot \exp (\zeta))
$$

defined whenever the right-hand side (i.e., $f(z)$ ) belongs to $\mathbb{C} \backslash \bar{D}$. Dynamical properties of $\mathcal{L}$ easily translate to properties of $f$ on the set of points whose orbits remain outside $\bar{D}$ forever. Our assumptions on $D$ imply that the function $\mathcal{L}$ is normalised in the sense of [Rem07a, RRRS11].

We occasionally cite results from other articles that are phrased in this language, but never use the logarithmic transform $\mathcal{L}$ directly in this article. Instead, we use the following terminology, which is inspired by this change of coordinates. See Figure 2(a).

Definition 2.2 (Relative cylindrical distance). For $z, w \in W_{0}$, we define the relative cylindrical distance $\operatorname{dist}_{\mathrm{cyl}}^{W_{0}}(z, w)$ to be the shortest cylindrical length of a curve $\gamma$ from $z$ to $w$ that is homotopic, in $\mathbb{C} \backslash \bar{D}$, to a curve in $W_{0}$.

Equivalently, if $U$ is a connected component of $\exp ^{-1}\left(W_{0}\right)$ and $\zeta, \omega$ are the logarithms of $z$ and $w$ that belong to $U$, then

$$
\operatorname{dist}_{\text {cyl }}^{W_{0}}(z, w)=|\zeta-\omega| \text {. }
$$

We similarly define the distance between two subsets of $W_{0}$, and the diameter $\operatorname{diam}_{\text {cyl }}^{W_{0}}$ with respect to this metric.

Since $f$ is expanding with respect to the cylindrical metric on $W_{0}$ by $(2.3)$, we have

$$
\operatorname{dist}_{\mathrm{cyl}}^{W_{0}}(f(z), f(w)) \geq 2 \cdot \operatorname{dist}_{\mathrm{cyl}}^{W_{0}}(z, w)
$$




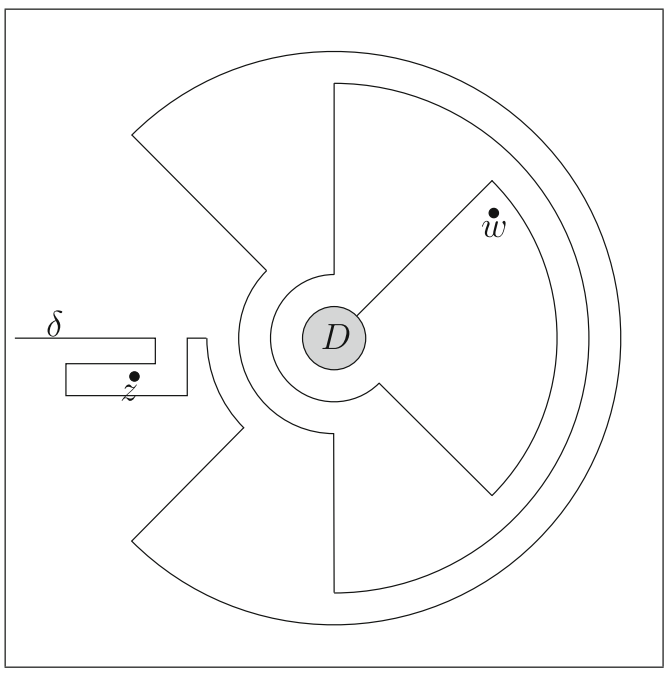

(a) Definition 2.2

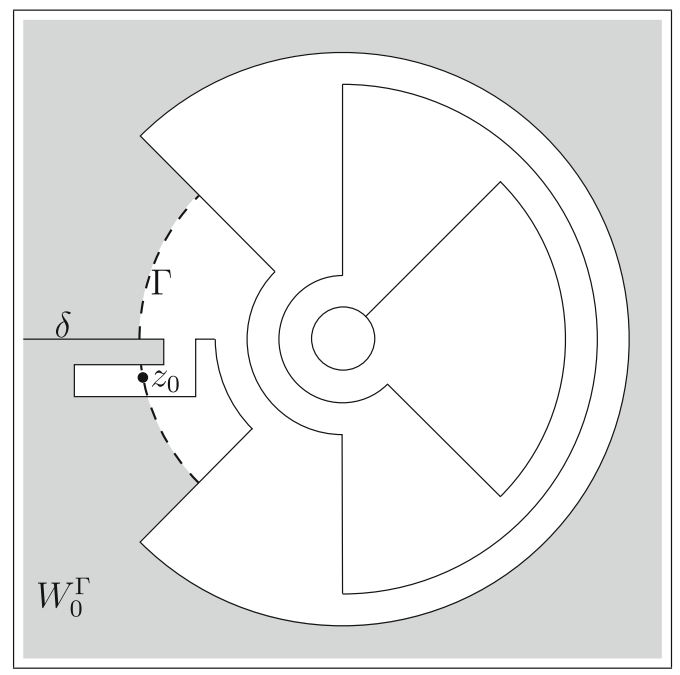

(b) Lemma 2.7

Figure 2: In (a), the cylindrical distance between $z$ and $w$ is less than $\pi$. However, any curve connecting $z$ and $w$ in $W_{0}$ takes more than two full turns around $D$, and hence the relative distance in the sense of Definition 2.2 is greater than $4 \pi$. The second image, in (b), illustrates Lemma 2.7 in this case. Here $\Gamma$ is a union of three cross-cuts of $W_{0}$ satisfying the conclusion of the lemma; the domain $W_{0}^{\Gamma}$ is shown shaded in grey. ( $\mathrm{Nb}$. the set $\Gamma$ constructed in the proof of Lemma 2.7 contains two additional cross-cuts; omitting these does not change the domain $W_{0}^{\Gamma}$, and therefore the conclusions of the lemma).

whenever $z$ and $w$ both belong to $\stackrel{\infty}{F}$ for some fundamental domain $F$.

Let us also note the following fact for future reference.

Observation 2.3. Let $\zeta_{0} \in W_{0}$. If $\left(z_{n}\right)_{n=0}^{\infty}$ is a sequence of points in $W_{0}$, then $\left|z_{n}\right| \rightarrow \infty$ if and only if dist ${ }_{\text {cyl }}^{W_{0}}\left(z_{n}, \zeta_{0}\right) \rightarrow \infty$.

External addresses and symbolic dynamics. The reason for introducing fundamental domains is that they can be used to assign symbolic dynamics to points whose orbit stays sufficiently large, and in particular to escaping points.

Definition 2.4 (External addresses). Let $f \in \mathcal{B}$, and let fundamental domains be defined as above. An (infinite) external address is a sequence $\underline{s}=F_{0} F_{1} F_{2} \ldots$ of fundamental domains of $f$. The address $\underline{s}$ is bounded if the set of fundamental domains occurring in $\underline{s}$ is finite; it is periodic if there is $k$ such that $F_{n+k}=F_{n}$ for all $n \geq 0$.

Let $\underline{s}=F_{0} F_{1} F_{2} \ldots$ be an external address, and recall that $\stackrel{\infty}{F}_{n}$ denotes the unbounded connected component of $F_{n} \backslash \bar{D}$. Then we define

$$
J_{\underline{s}}^{0}(f):=\left\{z \in \mathbb{C}: f^{n}(z) \in F_{n}^{\infty} \text { for all } n \geq 0\right\} .
$$

REMARK. For the purpose of this paper, we shall often use "address" synonymously with "external address". 
The main goal of this section is to prove the following.

Theorem 2.5 (Realisation of addresses). Let $\underline{s}$ be an external address.

(a) Suppose that $J_{\underline{s}}^{0}$ contains some point $z_{0}$. Then $J_{\underline{s}}^{0}$ also contains a closed unbounded connected set $X$ on which the iterates of $f$ tend to infinity uniformly. Moreover, dist $\mathrm{Cyl}_{\mathrm{c}}^{W_{0}}\left(z_{0}, X\right) \leq 4 \pi$.

(b) If $X_{1}$ and $X_{2}$ are unbounded, closed, connected subsets of $J_{\underline{s}}^{0}$ with $X_{1} \not \subset X_{2}$, then $X_{2} \subset X_{1}$ and $\left.f^{n}\right|_{X_{2}} \rightarrow \infty$ uniformly.

(c) If $\underline{s}$ is bounded, then $J_{\underline{s}}^{0} \neq \emptyset$. Furthermore, there exists $R>r(D)$, depending on the finite collection of fundamental domains occurring in $\underline{s}$ but not otherwise on $\underline{s}$, such that the set $X$ in (a) can be chosen to contain a point of modulus $R$.

(d) Conversely, if $\mathcal{F}$ is a finite collection of fundamental domains, then there is $R>0$ such that the iterates of $f$ tend to infinity uniformly on the closed set

$$
\bigcup_{\underline{s} \in \mathcal{F}^{N_{0}}} J_{\underline{s}}^{0} \backslash D_{R}(0)=\left\{z \in \mathbb{C}:|z| \geq R \text { and } f^{n}(z) \in \bigcup_{F \in \mathcal{F}} \bar{F} \text { for all } n \geq 0\right\} \text {. }
$$

This collection of results is not entirely new. Claims (a) and (b) are variants of Proposition 3.2 and Corollary 3.4 of [Rem07a]. Part (d) follows from [Rem08, Lemma 2.1]. Claim (c) is proved in [Rem08, Theorem 2.4] for fixed addresses, and the proof extends directly to the case of arbitrary bounded addresses; compare also [BF15, Proposition 2.11]. Alternatively, when $f$ is of disjoint type, $J_{\underline{s}} \neq \emptyset$ for bounded addresses $\underline{s}$ (and even for certain unbounded $\underline{s}$, see Proposition 2.13 below) by [BK07, Corollary B' on p. 405]; compare also [Rem16, Proposition 3.10]. Using the results of [Rem09], it can then be deduced for general functions in the class $\mathcal{B}$ that $J_{\underline{s}}^{0} \neq \emptyset$ for bounded $\underline{s}$.

Since the papers in question all use slightly different notation, we shall give a new proof of Theorem 2.5 that is self-contained and unified. We begin with a simple property of the set $J_{\underline{s}}^{0}$, which is similar to [EL92, Theorem 1].

LEMma 2.6 (One-dimensionality of $J_{\underline{s}}^{0}$ ). Let $\underline{s}=F_{0} F_{1} \ldots$ be an external address. Then $\overline{J_{\underline{s}}^{0}}$ is a subset of $J(f)$, has empty interior and does not separate the plane.

Proof. For each $n \geq 0, U_{n}:=\mathbb{C} \backslash \operatorname{cl}\left(\stackrel{\infty}{F}_{n}\right)$ is connected. Suppose, by contradiction, that $V_{0}$ was a connected component of $\mathbb{C} \backslash \partial J_{\underline{s}}^{0}$ that does not contain $U_{0}$. Then $V_{0} \subset F_{0}$, and $f: V_{0} \rightarrow f\left(V_{0}\right)$ is a conformal isomorphism.

It follows inductively that $f^{n}\left(V_{0}\right) \subset \stackrel{\infty}{F}_{n}$ for all $n \geq 0$. On the other hand, fix $z_{0} \in V_{0}$, and set $z_{n}:=f^{n}\left(z_{0}\right)$. It follows from the above that $f^{n}: V_{0} \rightarrow f^{n}\left(V_{0}\right)$ is univalent for all $n$. By (2.4) and the definition of the cylindrical derivative, we have $\left|\left(f^{n}\right)^{\prime}\left(z_{0}\right)\right| /\left|f^{n}\left(z_{0}\right)\right| \rightarrow \infty$ as $n \rightarrow \infty$. By Koebe's $1 / 4$-theorem it follows that $0 \in f^{n}\left(V_{0}\right)$ for sufficiently large $n$. This is a contradiction and proves that $\overline{J_{\underline{s}}^{0}}$ has empty interior and does not separate the plane. 
Let $z \in \overline{J_{s}^{0}}$. Suppose first that $\operatorname{dist}\left(f^{n}(z), D\right) \rightarrow \infty$ as $n \geq 0$. If $z$ belonged to the Fatou set of $f$, then it would follow from equicontinuity that there are $n_{0}$ and a neighbourhood $V$ of $z$ such that $f^{n}(V) \cap \bar{D}=\emptyset$ for all $n \geq n_{0}$. But then $f^{n_{0}}(V) \subset J_{\sigma^{n_{0}}(\underline{s})}^{0}$, and this contradicts the result we have just proved. So $z \in J(f)$.

Otherwise, there is a sequence $n_{k}$ such that $f^{n_{k}}(z) \nrightarrow \infty$. Then the spherical derivative of $f^{n_{k}}$ at $z$ is comparable to the corresponding cylindrical derivative. By (2.4), the latter tends to infinity as $k \rightarrow \infty$. Thus the family of iterates of $f$ is not normal at $z$ by Marty's theorem, and again $z \in J(f)$.

A separation lemma. We now formulate a key technical lemma - closely related to Lemmas 3.1 and 3.3 of [Rem07a] - that will be crucial for our unified proof of Theorem 2.5. Before making the formal statement, which is slightly technical, let us explain the idea. Let $z_{0} \in W_{0}$, and suppose that $z_{0}$ can be connected to infinity within the set of points of modulus greater than $R$. Then our lemma states (in particular) that no point $\zeta$ of modulus at most $R$ can be connected to infinity without passing near $z_{0}$, in the sense of relative cylindrical distance.

It may appear at first as though this is obvious, since the round circle centred at 0 and of modulus $\left|z_{0}\right|$ has cylindrical length $2 \pi$, and must intersect any curve connecting $\zeta$ to infinity. However, the diameter of the intersection of this circle with $W_{0}$ may be arbitrarily large when measured with respect to $\operatorname{dist}_{\mathrm{cyl}}^{W_{0}}$; see Figure 2.

Lemma 2.7 (Cross-cuts of. $W_{0}$ ). Let $z_{0} \in W_{0}$. Then there exists a union $\Gamma \ni z_{0}$ of cross-cuts of $W_{0}$ such that $\operatorname{dist}_{\text {cyl }}^{W_{0}}\left(z_{0}, \zeta\right) \leq 2 \pi$ for all $\zeta \in \Gamma$ and such that the unbounded connected component $W_{0}^{\Gamma}$ of $W_{0} \backslash \Gamma$ has the following property. If $R>0$ is such that $z_{0}$ belongs to the unbounded connected component of $W_{0} \backslash \overline{D_{R}(0)}$, then $W_{0}^{\Gamma}$ is also disjoint from $\overline{D_{R}(0)}$. Here $\Gamma$ can be chosen to consist only of arcs of the circle of radius $\left|z_{0}\right|$ centred at the origin.

Moreover, suppose that $A \subset W_{0}$ is any unbounded connected set with $z_{0} \in A$. Then, for all $z \in W_{0}^{\Gamma}$, $\operatorname{dist}_{\mathrm{cyl}}^{W_{0}}(z, A) \leq 2 \pi$.

REMARK. The curve $\delta$ in the definition of fundamental domains can be chosen to be piecewise analytic, in which case the number of cross-cuts in $\Gamma$ is necessarily finite. However, we do not require this.

Proof. Let $U$ be a connected component of $\exp ^{-1}\left(W_{0}\right)$; then $\exp : U \rightarrow W_{0}$ is a conformal isomorphism. For $\zeta \in U$, let $I_{\zeta}$ denote the vertical segment $\zeta+i \cdot[-2 \pi, 2 \pi]$. The fact that $U$ is disjoint from its $2 \pi i \mathbb{Z}$-translates implies the following separation property: if $\zeta_{0}, \zeta_{1} \in U$ with $\zeta_{0} \notin I_{\zeta_{1}}$, then either $I_{\zeta_{1}}$ separates $\zeta_{0}$ from infinity in $U$, or vice versa. (Compare [Rem07a, Lemma 3.3].)

Indeed, suppose otherwise. Then for $j=0,1$, there is a curve $\gamma_{j} \subset U$ connecting $\zeta_{j}$ to infinity, and not intersecting $I_{\zeta_{1-j}}$. Set $A:=\gamma_{0} \cup \gamma_{1} \cup\{\infty\}$, and let $a \in A$ be a point of minimal real part; say $a \in \gamma_{j}$. By [Rem16, Corollary 5.4], $I_{\zeta}$ separates $a$ from $\infty$ for every $\zeta \in A \backslash I_{\zeta}$. This is a contradiction to the fact that $\zeta_{1-j} \in A$, but $\gamma_{j}$ does not intersect $I_{\zeta_{1-j}}$. 
Now let $\zeta_{0}$ be the unique point of $\exp ^{-1}\left(z_{0}\right) \cap U$ and set $I:=I_{\zeta_{0}}$. Observe that the endpoints of $I$ are elements of $\exp ^{-1}\left(z_{0}\right)$ different from $\zeta_{0}$, and hence do not belong to $U$. We set $X:=I \cap U$; then $X$ is a collection of cross-cuts of $U$. Set $\Gamma:=\exp (X)$; we will prove the claims of the lemma by considering the unbounded connected component $V_{1}$ of $U \backslash I$. In other words, $V_{1}$ consists of all points of $U$ that are not separated from $\infty$ by $I_{\zeta}$.

By the separation property, for all $\zeta \in V_{1}, I_{\zeta}$ separates $\zeta_{0}$ from infinity in $U$. So if $A \subset U$ is an unbounded connected set with $\zeta_{0} \in A$, then $I_{\zeta} \cap A \neq \emptyset$, and in particular $\operatorname{dist}(\zeta, A) \leq 2 \pi$. Moreover, by hypothesis $\zeta_{0}$ can be connected to infinity by a curve in $U$ that stays at real parts greater than $\log R$. So $\operatorname{Re} \zeta>\log R$ for all $\zeta \in V_{1}$.

Since $\exp \left(V_{1}\right)=W_{0}^{\Gamma}$, this completes the proof of the lemma.

Existence of unbounded sets of escaping points. With these preparations, we are now able to prove the existence of unbounded connected subsets of $J_{\underline{s}}^{0}$ under very general hypotheses.

Theorem 2.8 (Unbounded subsets of $J_{\underline{s}}^{0}$ ). Let $\underline{s}=F_{0} F_{1} F_{2} \ldots$ be an external address. Suppose that $\left(z_{n}\right)_{n=0}^{\infty}$ is a sequence of points such that each $z_{n} \in \stackrel{\infty}{F}_{n}$ for all $n \geq 0$.

Suppose furthermore that there is $C>0$ such that $\operatorname{dist}_{\mathrm{cyl}}^{W_{0}}\left(z_{n},\left.f\right|_{F_{n}} ^{-1}\left(z_{n+1}\right)\right) \leq C$ for all $n \geq 0$ for which $\left(\left.f\right|_{F_{n}}\right)^{-1}\left(z_{n+1}\right) \notin \stackrel{\infty}{F_{n}}$.

Then there is a closed, unbounded and connected subset $X \subset J_{\underline{s}}^{0}$ such that $\operatorname{dist}_{\text {cyl }}^{W_{0}}\left(z_{0}, X\right) \leq 2 \max (2 \pi, C)$.

Proof. For $n \geq 0$, let $\Gamma_{n}$ be the union of cross-cuts from Lemma 2.7, applied to $z_{n}$. Let $A_{n}^{k}$ be a sequence of unbounded closed connected sets, defined for $n, k \geq 0$ as follows. Let $A_{n}^{0}$ be the closure of the unbounded connected component of $F_{n} \backslash \Gamma_{n}$. For $k \geq 0$, let $A_{n}^{k+1}$ be the closure of the unbounded connected component of $\left(\left.f\right|_{F_{n}}\right)^{-1}\left(A_{n+1}^{k}\right) \backslash \Gamma_{n}$. Observe that $A_{n}^{k+1} \subset A_{n}^{k}$ for all $n$ and $k$.

Claim. dist $_{\text {cyl }}^{W_{0}}\left(A_{n}^{k}, z_{n}\right) \leq 2 \max (2 \pi, C)$ for all $n, k \geq 0$.

Proof. Since $A_{n}^{0}$ intersects $\Gamma_{n}$, the claim is true for $k=0$. Suppose that $k \geq 0$ is such that the claim holds for all $n \geq 0$. Let $n \geq 0$, and set $B:=\left(\left.f\right|_{F_{n}}\right)^{-1}\left(A_{n+1}^{k}\right)$. If $B \cap \Gamma_{n} \neq \emptyset$, then $A_{n}^{k+1}$ intersects $\Gamma_{n}$ and the claim is immediate from the properties of $\Gamma_{n}$. Otherwise, $A_{n}^{k+1}=B$. Suppose first that $\left.\left(\left.f\right|_{F_{n}}\right)^{-1}\left(z_{n+1}\right)\right) \notin \stackrel{\infty}{F}_{n}$. Then

$$
\begin{aligned}
\operatorname{dist}_{\mathrm{cyl}}^{W_{0}}\left(A_{n}^{k+1}, z_{n}\right) & \leq \operatorname{dist}_{\mathrm{cyl}}^{W_{0}}\left(A_{n}^{k+1},\left(\left.f\right|_{F_{n}}\right)^{-1}\left(z_{n+1}\right)\right)+C \\
& \leq \max (2 \pi, C)+C \leq 2 \max (2 \pi, C)
\end{aligned}
$$

by (2.4). Now suppose that $\left.\left(\left.f\right|_{F_{n}}\right)^{-1}\left(z_{n+1}\right)\right) \in \stackrel{\infty}{F_{n}}$. By the inductive hypothesis, and since $\Gamma_{n}$ separates $\partial D$ from $B$, we can connect $B$ to $\Gamma_{n}$ by a curve $\gamma$ with $\operatorname{diam}_{\mathrm{cyl}}^{W_{0}}(\gamma) \leq \max (2 \pi, C)$. Since $\operatorname{diam}_{\mathrm{cyl}}^{W_{0}}\left(\Gamma_{n}\right) \leq 2 \pi$, the claim follows. 
Now set $X_{1}:=\bigcap_{k \geq 0} A_{0}^{k}$. Then $X_{1} \cup\{\infty\}$ is compact and connected as a countable intersection of compact connected sets. Moreover, $X_{1}$ contains a point $\zeta$ with $\operatorname{dist}_{\text {cyl }}^{W_{0}}\left(\zeta, z_{0}\right) \leq 2 \max (\pi, C)$. If $X$ is the connected component of $X_{1}$ containing $\zeta$, then $X$ is unbounded by the boundary bumping theorem [Nad92, Theorem 5.6], and the proof is complete.

In particular, we obtain the following partial results towards Theorem 2.5.

Corollary 2.9 (Realised addresses have unbounded sets). Suppose that $\underline{s}$ is an external address, and that there is a point $z_{0} \in J_{\underline{s}}^{0}$. Then $J_{\underline{s}}^{0}$ contains an unbounded closed connected set $X$, and dist ${ }_{\text {cyl }}^{W_{0}}\left(z_{0}, X\right) \leq 4 \pi$.

Proof. Set $z_{n}:=f^{n}\left(z_{0}\right)$, and apply Theorem 2.8 .

Corollary 2.10 (Bounded addresses are realised). Suppose that $\mathcal{F}$ is a finite collection of fundamental domains. Then there is $R>0$ with the following property.

If $\underline{s}$ is an external address whose entries are all in $\mathcal{F}$, then $J_{s}^{0}$ contains an unbounded connected set $X$ which contains a point of modulus at most $R$.

Proof. Pick a base-point $\zeta_{0} \in W_{0}$. For $F \in \mathcal{F}$, let $\zeta_{F}$ be the preimage of $\zeta_{0}$ in $F$. Then there is a constant $C$ such that $\operatorname{dist}_{\text {cyl }}^{W_{0}}\left(\zeta_{0}, \zeta_{F}\right) \leq C$ for all $F \in \mathcal{F}$ (when defined).

If $\underline{s}$ is an external address in $\mathcal{F}^{\mathbb{N}_{0}}$, we can set $z_{n}:=\zeta_{0}$ for all $n \geq 0$, and apply Theorem 2.8. We obtain an unbounded connected set $X$ with $\operatorname{dist}_{\text {cyl }} \bar{W}_{0}\left(\zeta_{0}, X\right)$ $\leq 2 \max (2 \pi, C)$. If $R$ is sufficiently large (depending only on $C$ ), then $X$ contains a point of modulus at most $R$.

Uniform escape to infinity. To complete the proof of Theorem 2.5, we consider the question of uniform escape to infinity on an unbounded connected subset of $J_{\underline{s}}^{0}$. Recall that by definition of $J_{\underline{s}}^{0}$ a point $z$ belongs to $J_{\underline{s}}^{0}$ if and only if $z_{n} \in \underline{F}_{n}$ for all $n$, where $\underline{s}=F_{0} F_{1} \ldots$

Lemma 2.11 (Uniform escape on unbounded connected sets). Let $\underline{s}=F_{0} F_{1} F_{2} \ldots$ be an external address, and suppose that $X \subset J_{s}^{0}$ is unbounded and connected. Furthermore, assume that there is a sequence $\left(z_{n}\right)_{n=0}^{\infty}$ (not necessarily an orbit of $f)$ such that $z_{n} \in \stackrel{\infty}{F}_{n}$, and such that $\operatorname{dist}_{\text {cyl }}^{W_{0}}\left(z_{n}, f^{n}(X)\right) \rightarrow \infty$. Then $\left.f^{n}\right|_{\bar{X}} \rightarrow \infty$ uniformly.

Proof. Let $\zeta_{0} \in W_{0}$ be any base point. We may assume that $\operatorname{dist}_{\text {cyl }}^{W_{0}}\left(z_{n}, \zeta_{0}\right) \rightarrow \infty$. Indeed, set $\eta_{n}:=\operatorname{dist}_{\text {cyl }}^{W_{0}}\left(z_{n}, f^{n}(X)\right)$ and let $\left(\xi_{n}\right)_{n=0}^{\infty}$ be any sequence in $W_{0}$ with

$$
\operatorname{dist}_{\text {cyl }}^{W_{0}}\left(\xi_{m}, \zeta_{0}\right) \rightarrow \infty \quad \text { and } \quad \operatorname{dist}_{\text {cyl }}^{W_{0}}\left(\xi_{m}, \zeta_{0}\right) \leq \eta_{n} / 3
$$

Now define

$$
\tilde{z}_{n}:= \begin{cases}z_{n} & \text { if } \text { dist }_{\text {cyl }}^{W_{0}}\left(z_{n}, \zeta_{0}\right)>\eta_{n} / 3 \\ \xi_{n} & \text { otherwise. }\end{cases}
$$


Clearly $\operatorname{dist}\left(\tilde{z}_{n}, \zeta_{0}\right) \rightarrow \infty$ and $\operatorname{dist}_{\text {cyl }}^{W_{0}}\left(\tilde{z}_{n}, f^{n}(X)\right) \geq \eta_{n} / 3 \rightarrow \infty$, as desired.

Let $\Gamma_{n}$ be the union of cross-cuts associated to $z_{n}$ by Lemma 2.7. Then, for sufficiently large $n, f^{n}(\bar{X})$ is disjoint from $\Gamma_{n}$, and hence belongs to $W_{0}^{\Gamma_{n}}$. Since $\left|z_{n}\right| \rightarrow \infty$ by Observation 2.3, it follows that $f^{n}(\bar{X}) \rightarrow \infty$ uniformly, as required.

Proof of Theorem 2.5. We first prove (b), so let $X_{1}, X_{2} \subset J_{\underline{s}}^{0}$ be closed, unbounded and connected with $X_{1} \not \subset X_{2}$.

Let $z_{0} \in X_{1} \backslash X_{2}$ and set $z_{n}:=f^{n}\left(z_{0}\right)$. Then $\operatorname{dist}\left(z_{n}, f^{n}\left(X_{2}\right)\right) \rightarrow \infty$ by (2.4). In particular, $\left.f^{n}\right|_{X_{2}} \rightarrow \infty$ uniformly by Lemma 2.11 .

Let $n_{0}$ be sufficiently large that $\operatorname{dist}_{\text {cyl }}^{W_{0}}\left(z_{n}, f^{n}\left(X_{2}\right)\right)>2 \pi$ for $n \geq n_{0}$. Let $\Gamma_{n}$ be the union of crosscuts associated to $z_{n}$ by Lemma 2.7. Then $f^{n}\left(X_{2}\right) \subset W_{0}^{\Gamma_{n}}$ for $n \geq n_{0}$. Since $f^{n}\left(X_{1}\right)$ connects $z_{n}$ to $\infty$, we have $\operatorname{dist}\left(f^{n}\left(X_{1}\right), f^{n}(\zeta)\right) \leq 2 \pi$ for all $\zeta \in X_{2}$ and all $n \geq 0$. Again by (2.4), we have $\operatorname{dist}\left(X_{1}, \zeta\right)=0$, and hence $\zeta \in X_{1}$, as required.

Now let us prove (a), so suppose that $z_{0} \in J_{s}^{0}$. By Corollary 2.9, there is an unbounded closed connected set $X \subset J_{\underline{s}}^{0}$ with dist ${ }_{\text {cyl }}^{W_{0}}\left(X, z_{0}\right) \leq 4 \pi$. We may assume that $z_{0} \notin X$. Indeed, otherwise we let $\varepsilon$ be sufficiently small and replace $X$ by an unbounded connected component of $X \backslash D_{\varepsilon}\left(z_{0}\right)$ that intersects $\partial D_{\varepsilon}\left(z_{0}\right)$.

Now set $z_{n}:=f^{n}\left(z_{0}\right)$. By $(2.4)$, we have $\operatorname{dist}_{\text {cyl }}^{W_{0}}\left(f^{n}(X), z_{n}\right) \rightarrow \infty$, and hence it follows from Lemma 2.11 that $\left.f^{n}\right|_{X} \rightarrow \infty$ uniformly. This completes the proof of (a) of Theorem 2.5.

Part (c) follows directly from Corollary 2.10. To prove (d), observe first that equality of the sets in (2.5) holds as soon as $R$ is sufficiently large. Indeed, suppose that $F, F^{\prime} \in \mathcal{F}$ and that $z \in \bar{F}$ maps to some point in $\overline{F^{\prime}}$ of modulus at least $R$. Since $f(z) \in W_{0}$, we must in fact have $z \in F$, and additionally $z \in \stackrel{\infty}{F}$ if $R$ is sufficiently large.

Now let $\zeta_{0}$ and $\left(\zeta_{F}\right)_{F \in \mathcal{F}}$ be defined as in the proof of Corollary 2.10. Let $R$ be sufficiently large such that any point $z \in W_{0}$ of modulus at least $R$ satisfies $\operatorname{dist}_{\text {cyl }}^{W_{0}}\left(\zeta_{0}, z\right) \geq 3 \max (C, 2 \pi)$.

Let $z$ be a point whose orbit is contained in $\bigcup_{F \in \mathcal{F}} \bar{F}$, and furthermore $|z| \geq R$. Then it follows that

$$
\operatorname{dist}_{\text {cyl }}^{W_{0}}\left(\zeta_{0}, f(z)\right) \geq 2 \cdot\left(\operatorname{dist}_{\text {cyl }}^{W_{0}}\left(\zeta_{0}, z\right)-\max (C, 2 \pi)\right) \geq \frac{4}{3} \operatorname{dist}_{\text {cyl }}^{W_{0}}\left(\zeta_{0}, z\right) .
$$

It follows by induction that $\operatorname{dist}_{\text {cyl }}^{W_{0}}\left(\zeta_{0}, f^{n}(z)\right) \rightarrow \infty$ uniformly in $n$, and the claim follows.

Exponentially bounded addresses. As noted above, the results on bounded addresses can be generalised to certain unbounded addresses. While we do not require this fact for this paper, we shall record it for future reference.

Definition 2.12 (Exponentially bounded addresses). Let $\zeta_{0} \in W_{0}$ be an arbitrary base point. For any fundamental domain $F$, let $\zeta_{F}$ be the unique preimage of $\zeta_{0}$ in $F$. 
We say that an infinite external address $\underline{s}$ is exponentially bounded if there exists a positive real number $T$ with the following property. For all $n \geq 0$, if $\zeta_{F_{n}} \in \stackrel{\infty}{F}_{n}$, then

$$
\operatorname{dist}_{\text {cyl }}^{W_{0}}\left(\zeta_{0}, \zeta_{F_{n}}\right) \leq \exp ^{n}(T)
$$

REMARK. If $\xi$ is another base-point, then it follows from 2.4 that $\operatorname{dist}_{\text {cyl }}^{W_{0}}\left(\zeta_{F}, \xi_{F}\right)$ is uniformly bounded (where defined) for all fundamental domains $F$. Thus it follows that the definition of exponentially bounded addresses is independent of the choice of base point $\zeta_{0}$.

Exponentially bounded addresses were defined previously for exponential maps [SZ03a] and cosine maps [RS08b]; it is easy to see that in these cases the definition agrees with ours. For these families, the class of exponentially bounded addresses $\underline{s}$ agrees precisely with those for which $J_{\underline{s}}^{0} \neq \emptyset$. This is no longer true for general $f$, even when $f$ has finite order of growth; see $[\mathrm{ABR}]$.

In [BK07, Corollary B'], it is shown that $J_{\underline{s}}^{0} \neq \emptyset$ for a certain class of addresses, namely those whose orbits remain within finitely many tracts, and such that the "index" of the corresponding fundamental domains within each tract does not grow faster than an iterated exponential. It is easy to see that such addresses are exponentially bounded in our sense, but the converse is not the case. (For example, our definition allows for addresses taking values in fundamental domains that lie in infinitely many different tracts.)

We now show that (c) and (d) of Theorem 2.5 can be extended to exponentially bounded addresses as follows.

Proposition 2.13 (Exponentially bounded addresses are realised). Let $\underline{s}$ be an exponentially bounded address. Then $J_{s}^{0} \neq \emptyset$.

More precisely, there is a number $R>0$, depending only on the base-point $\zeta_{0} \in W_{0}$ and $T>0$, with the following property. If $\underline{s}$ is exponentially bounded for this choice of $\zeta_{0}$ and $T$, then $J_{s}^{0}$ contains an unbounded connected set $X$ on which the iterates tend to infinity uniformly, and which contains a point of modulus $R$.

Conversely, the iterates of $f$ tend to infinity uniformly on

$$
\bigcup_{\underline{s}} J_{\underline{s}}^{0} \backslash D_{R}(0)
$$

where the union is taken over all external addresses $\underline{s}$ as above.

Proof. We shall use an expansion estimate [RRRS11, Lemma 3.1], which is stronger than (2.4) at large distances. (Compare also [BK07, Lemma 3.3].) We will use this estimate in the following form, which follows easily from the version stated in [RRRS11]: There are constants $C_{1}, C_{2}>0$ with the following property. If $F$ is a fundamental domain and $\zeta_{1}, \zeta_{2} \in \stackrel{\infty}{F}$ with $\operatorname{dist}_{\text {cyl }}^{W_{0}}\left(\zeta_{1}, \zeta_{2}\right) \geq C_{1}$, then

$$
\operatorname{dist}_{\mathrm{cyl}}^{W_{0}}\left(f\left(\zeta_{1}\right), f\left(\zeta_{2}\right)\right) \geq \exp \left(C_{2} \cdot \operatorname{dist}_{\mathrm{cyl}}^{W_{0}}\left(\zeta_{1}, \zeta_{2}\right)\right)
$$


Now fix $\zeta_{0}$ and $T>0$, and denote by $\mathcal{S}$ the set of all addresses that satisfy Definition 2.12 for these choices. We may assume without loss of generality that $\zeta_{0}$ is chosen sufficiently large to ensure that $\zeta_{F} \in \stackrel{\infty}{F}$ for every fundamental domain $F$. (To this end, we may need to increase $T$, but only by a finite amount according to the remark following Definition 2.12.) Define $E(t):=\exp \left(C_{2} \cdot t\right)$. By basic properties of exponential growth, there is $\tilde{R}>0$ such that the following hold for all $x \geq \tilde{R} / 3$.

$$
\begin{aligned}
E(x) & >3 E(x / 2)>E(x / 2)>2 x, \quad \text { and } \\
E^{n}(x) & \geq E^{n}(\tilde{R} / 3)>\exp ^{n}(T)+2 \pi .
\end{aligned}
$$

We choose $R$ sufficiently large to ensure that $\operatorname{dist}_{\text {cyl }}^{W_{0}}\left(z, \zeta_{0}\right)>\tilde{R}$ whenever $|z| \geq R$. that

Suppose that $\underline{s} \in \mathcal{S}$, and let $z \in J_{\underline{s}}^{0}$ such that $t:=\operatorname{dist}_{\text {cyl }}^{W_{0}}\left(z, \zeta_{0}\right) \geq \tilde{R}$. We claim

$$
\operatorname{dist}_{\mathrm{cyl}}^{W_{0}}\left(f^{n}(z), \zeta_{0}\right) \geq 3 E^{n}(t / 3) .
$$

Indeed, this is trivial for $n=0$, and if the claim holds for $n$, then

$$
\operatorname{dist}_{\mathrm{cyl}}^{W_{0}}\left(f^{n}(z), \zeta_{F_{n}}\right) \geq \operatorname{dist}_{\mathrm{cyl}}^{W_{0}}\left(f^{n}(z), \zeta_{0}\right)-\exp ^{n}(T) \geq 2 E^{n}(t / 3) .
$$

Hence, by (2.8) and 2.9,

$$
\operatorname{dist}_{\mathrm{cyl}}^{W_{0}}\left(f^{n+1}(z), \zeta_{0}\right)=\operatorname{dist}_{\mathrm{cyl}}^{W_{0}}\left(f^{n+1}(z), f\left(\zeta_{F_{n}}\right)\right) \geq E\left(2 E^{n}(t / 3)\right) \geq 3 E^{n+1}(t / 3) .
$$

The claim follows by induction. In particular,

$$
\operatorname{dist}_{\mathrm{cyl}}^{W_{0}}\left(f^{n}(z), \zeta_{0}\right) \geq 3 E^{n}(\tilde{R} / 3) \geq \exp ^{n}(T) .
$$

Since this applies to all points in the union (2.7), the second claim of the proposition follows.

Now let us prove the first. Set $t:=\tilde{R} / 3$. For $n \geq 0$, choose $z_{n} \in \stackrel{\infty}{F}_{n}$ with $\operatorname{dist}_{\text {cyl }}^{W_{0}}\left(z_{n}, \zeta_{0}\right)=E^{n}(t)$. Such a point exists because $\operatorname{dist}\left(\zeta_{F_{n}}, \zeta_{0}\right)<E^{n}(t)$, and $\zeta_{F_{n}}$ can be connected to infinity within $\stackrel{\infty}{F_{n}}$.

For $n \geq 0$, let $\Gamma_{n}$ be the union of cross-cuts from Lemma 2.7, applied to $z_{n}$, and let $A_{n}^{k}$, for $n, k \geq 0$, be defined precisely as in the proof of Theorem 2.8.

Claim. $\operatorname{dist}_{\mathrm{cyl}}^{W_{0}}\left(A_{n}^{k}, \zeta_{0}\right) \leq 3 E^{n}(t)$ for all $n, k \geq 0$.

Proof. If $A_{n}^{k} \cap \Gamma_{n} \neq \emptyset$, then the claim follows by choice of $z_{n}$. In particular, this is always the case for $k=0$.

So suppose that $k \geq 0$ is such that the claim holds for all $n \geq 0$, and that $n$ is such that $A_{n}^{k+1} \cap \Gamma_{n}=\emptyset$. Then, by definition, $f\left(A_{n}^{k+1}\right)=A_{n+1}^{k}$. By the inductive hypothesis, the latter contains a point $w$ with $\operatorname{dist}_{\mathrm{cyl}}^{W_{0}}\left(w, \zeta_{0}\right) \leq 3 E^{n+1}(t)$. Define $\tilde{w}:=\left(\left.f\right|_{F_{n}}\right)^{-1}(w) \in A_{n+1}^{k}$. Then, by (2.8) and (2.9),

$$
\operatorname{dist}_{\mathrm{cyl}}^{W_{0}}\left(\tilde{w}, \zeta_{F_{n}}\right) \leq E^{-1}\left(3 E^{n+1}(t)\right) \leq 2 E^{n}(t) .
$$


So

$$
\operatorname{dist}_{\text {cyl }}^{W_{0}}\left(\tilde{w}, \zeta_{0}\right) \leq \operatorname{dist}_{\text {cyl }}^{W_{0}}\left(\tilde{w}, \zeta_{F_{n}}\right)+\operatorname{dist}_{\text {cyl }}^{W_{0}}\left(\zeta_{F_{n}}, \zeta_{0}\right) \leq 2 E^{n}(t)+\exp ^{n}(T) \leq 3 E^{n}(t)
$$

by (2.10), as claimed.

In particular, the set $\bigcap_{k>0} A_{0}^{k}$ has a connected component $X$ containing a point with $\operatorname{dist}\left(A_{n}^{k}, \zeta_{0}\right) \leq 3 t=\tilde{R}$, and hence of modulus at most $R$. Since $X$ is connected and unbounded, it also contains a point of modulus exactly $R$. The proof is complete.

\section{Hyperbolic Expansion and Fundamental Tails}

For the remainder of the article, we shall specialise to the case where our transcendental entire function $f$ has bounded postsingular set $\mathcal{P}(f)$.

This section collects some fundamental preliminary material concerning these functions. We begin by noting a global expansion property away from the postsingular set, and then proceed to introduce the notation of fundamental tails, which will later be used to study the structure of the set of escaping points.

Recall that if $\Omega$ is a hyperbolic domain, we denote by $\rho_{\Omega}$ the density of the hyperbolic metric on $\Omega$.

Proposition 3.1 (Hyperbolic expansion). For every transcendental entire function $f, \# \mathcal{P}(f) \geq 2$.

Now suppose that $\mathcal{P}(f)$ is bounded, let $\mathcal{P}$ be a compact forward-invariant set with $\mathcal{P}(f) \subset \mathcal{P}$, and let $\Omega$ be the unbounded connected component of $\mathbb{C} \backslash \mathcal{P}$. If $z \in V:=f^{-1}(\Omega) \subset \Omega$, then $f$ is strictly expanding at $z$ in the hyperbolic metric of $\Omega$. Moreover, this expansion factor tends to infinity as $z \rightarrow \infty$ in $V$. That is,

$$
\|\mathrm{D} f(z)\|_{\Omega}:=\left|f^{\prime}(z)\right| \cdot \frac{\rho_{\Omega}(f(z))}{\rho_{\Omega}(z)}>1,
$$

and

$$
\|\mathrm{D} f(z)\|_{\Omega} \rightarrow \infty \quad \text { as } \quad|z| \rightarrow \infty
$$

In particular, for every $\varepsilon>0$ there is $\Lambda>1$ such that $\|\operatorname{D} f(z)\|_{\Omega} \geq \Lambda$ whenever $z \in V$ with $\operatorname{dist}(z, \mathcal{P}) \geq \varepsilon$.

This result was proved, for a hyperbolic entire function $f$ and a certain choice of $\mathcal{P}$, in [Rem09, Lemma 5.1]. The same proof goes through whenever $\mathcal{P}$ intersects the unbounded connected component of $\mathbb{C} \backslash S(f)$. This can always be ensured by adding a periodic orbit to $\mathcal{P}$ that intersects this component, which is sufficient for all our purposes. However, for completeness and future reference, we shall prove the result for general $\mathcal{P}$, with a slightly simpler proof than that given in [Rem09]. To do so, we use the following simple fact. 
LEMMA 3.2 (Preimages in annuli). Let $f$ be an entire transcendental function which is bounded on an unbounded connected set. Let $z_{1}, z_{2} \in \mathbb{C}$. Then, for all $C>1$, and all sufficiently large $R, f^{-1}\left(\left\{z_{1}, z_{2}\right\}\right)$ contains a point of modulus between $R / C$ and $C \cdot R$.

Proof. Let $C>1$ and set $A:=\{z \in \mathbb{C}: 1 / C<|z|<C\}$. Suppose by contradiction that $R_{n} \rightarrow \infty$ is a sequence such that the functions

$$
g_{n}: A \rightarrow \mathbb{C} ; \quad z \mapsto f\left(R_{n} \cdot z\right)
$$

omit both $z_{1}$ and $z_{2}$. By Montel's theorem, this sequence of functions is normal, and hence converges locally uniformly, possibly after restricting to a subsequence. By assumption, $\lim \sup \min _{|z|=1} g_{n}(z)<\infty$, and hence the limit function is holomorphic. But this implies that $f$ remains bounded on the circle of radius $R_{n}$ as $n \rightarrow \infty$. Hence $f$ is bounded by the maximum principle and hence constant by Liouville's theorem, a contradiction.

Proof of Proposition 3.1. The fact that $\# \mathcal{P}(f) \geq 2$ is well-known: Otherwise, $f$ would be a self-covering of a punctured plane, and hence conformally conjugate to $z \mapsto z^{d}$ for some $d$. However, $f$ is transcendental.

So $\Omega$ is indeed a hyperbolic domain. Since $f: V \rightarrow \Omega$ is a covering map, and hence a local isometry with respect to the hyperbolic metrics of $V$ and $\Omega$, we have $\rho_{V}(z)=\rho_{\Omega}(f(z))\left|f^{\prime}(z)\right|$ for all $z \in V$.

The open mapping theorem implies that every connected component of the set $V=f^{-1}(\Omega)$ is unbounded (compare e.g. [AR17, Lemma 4.3]). Hence, by forwardinvariance of $\mathcal{P}$, we have $V \subset \Omega$.

Since $\# \mathcal{P} \geq 2$, and by Picard's theorem, $\mathbb{C} \backslash V=\mathbb{C} \backslash f^{-1}(\mathcal{P})$ contains points of arbitrarily large modulus. Hence $V \subsetneq \Omega$. By Pick's theorem, it follows that

$$
\|\mathrm{D} f(z)\|_{\Omega}=\left|f^{\prime}(z)\right| \cdot \frac{\rho_{\Omega}(f(z))}{\rho_{\Omega}(z)}=\frac{\rho_{V}(z)}{\rho_{\Omega}(z)}>1
$$

for all $z \in V$. This establishes the first claim.

Furthermore, since $f \in \mathcal{B}$, there is an unbounded connected set on which $f$ is bounded. (For example, the boundary of one of the tracts of $f$ ). By Lemma 3.2, there is a sequence $\left(c_{n}\right)_{n \geq 0}$ in $\Omega \backslash V$ such that $c_{n} \rightarrow \infty$ and $\left|c_{n+1} / c_{n}\right| \leq 2$. (We can even ensure $\left|c_{n+1} / c_{n}\right| \rightarrow 1$ by letting the constant $C$ in Lemma 3.2 tend to 1 , but do not require this here.) Hence $1 / \rho_{V}(z)=O(|z|)$ as $z \rightarrow \infty$ in $V$. (Compare [Rem09, Lemma 2.1] and see also [MR13, Proposition 3.4] and [Min17].) On the other hand, $\rho_{\Omega}(z)=O(1 /(|z| \log |z|))$ as $z \rightarrow \infty$. The claim follows.

Corollary 3.3 (Sets remaining in $\Omega$ ). Let $f \in \mathcal{B}$. Let $\Omega, \mathcal{P}$ be as in Proposition 3.1, and suppose that $U \subset \Omega$ is open with $f^{n}(U) \subset \Omega$ for all $n$. Then $\operatorname{dist}\left(f^{n}(z), \mathcal{P}\right) \rightarrow 0$ uniformly on compact subsets of $U$.

In particular, if $z_{0} \in \Omega$ with $f^{n}\left(z_{0}\right) \in \Omega$ for all $n$, and $\lim \sup \operatorname{dist}\left(f^{n}\left(z_{0}\right), \mathcal{P}\right)>0$, then $z_{0} \in J(f)$. 
Proof. Note that $\left(\left.f^{n}\right|_{U}\right)$ form a normal family by Montel's theorem, so $U \subset F(f)$. Therefore the first claim follows from the second: if $\operatorname{dist}\left(f^{n}(z), \mathcal{P}\right) \rightarrow 0$ for all $z \in U$, then this convergence is automatically uniform on compact subsets of $U$.

So suppose that $z_{0} \in \Omega$, and that there exist $\varepsilon>0$ and an increasing sequence $\left(n_{k}\right)_{k=0}^{\infty}$ such that $\operatorname{dist}\left(f^{n_{k}}\left(z_{0}\right), \mathcal{P}\right) \geq \varepsilon$. We must show that $z_{0} \in J(f)$.

It follows from Lemma 3.2 that $\operatorname{dist}_{\Omega}\left(z, f^{-1}(\mathcal{P})\right)$ remains bounded as $z \rightarrow \infty$ in $\Omega$. Hence

$$
\delta:=\sup _{k} \operatorname{dist}_{\Omega}\left(f^{n_{k}}\left(z_{0}\right), f^{-1}(\mathcal{P})\right)<\infty .
$$

So we can connect $f^{n_{k}}\left(z_{0}\right)$ to a point of $f^{-1}(\mathcal{P})$ by a curve $\gamma_{k}$ of hyperbolic length at most $\delta$ in $\Omega$. Pulling back $\gamma_{k}$ under $f^{n_{k}}$, we obtain a curve $\alpha_{k}$ connecting $z_{0}$ to a point $w_{k} \in f^{-\left(n_{k}+1\right)}(\mathcal{P})$. By Proposition 3.1, each of the curves $\alpha_{k}^{j}=f^{n_{j}}\left(\alpha_{k}\right)$, for $j<k$, has hyperbolic length at most $\delta$. Hence these curves stay a uniform distance away from $\mathcal{P}$, and again by Proposition 3.1, there is $\lambda>1$ such that

$$
\|\mathrm{D} f(z)\| \geq \lambda
$$

for all $k$, all $j<k$, and all $z \in \alpha_{k}^{j}$.

So in fact $\alpha_{k}$ has hyperbolic length at most $\delta \cdot \lambda^{k}$, and $w_{k} \rightarrow z_{0}$ as $k \rightarrow \infty$. converging to $z_{0}$. But $f^{n_{k+1}}\left(w_{k}\right) \in \mathcal{P}$, and $\operatorname{dist}\left(f^{n_{k_{1}}}\left(z_{0}\right), \mathcal{P}\right) \geq \varepsilon$. Therefore the family of iterates of $f$ is not equicontinuous at $z_{0}$, and $z_{0} \in J(f)$, as desired.

REMARK. The result can also be proved by appealing to the classification of Fatou components.

Fundamental tails. Let $f$ be an entire transcendental function with bounded postsingular set, and denote the unbounded connected component of $\mathbb{C} \backslash \mathcal{P}(f)$ by $\Omega$. (In everything that follows, we could more generally let $\Omega$ be as in Proposition 3.1; i.e. the unbounded connected component of $\mathbb{C} \backslash \mathcal{P}$ where $\mathcal{P}$ is a forward-invariant compact set containing $\mathcal{P}(f)$. However, we shall not require this extra generality.)

Let $D$ and $\gamma$ be as in Section 2. We may additionally assume that $D$ is chosen sufficiently large to ensure that $\mathcal{P}(f) \subset D$ and that

$$
\|\mathrm{D} f(z)\|_{\Omega} \geq 2
$$

whenever $f(z) \notin D$ (recall (3.2)).

Recall that fundamental domains are the connected components of the preimage of $W_{0}=\mathbb{C} \backslash(\bar{D} \cup \delta)$ under $f$. All concepts that follow depend a priori on this choice of fundamental domains, i.e. on the choice of the initial configuration consisting of $D$ and $\delta$. However, it turns out that this choice is not essential. (Compare Observation 4.12.)

The postsingular set $\mathcal{P}(f)$ is contained in $D$, and the image of any fundamental domain is contained in $\mathbb{C} \backslash \bar{D}$. Hence the closure of a fundamental domain does not intersect the postsingular set. It follows that for any $n \geq 0$, any fundamental domain 
$F$, and any connected component $\tau$ of $f^{-n}(F), \tau$ is a Jordan domain on the Riemann sphere whose boundary contains $\infty$ and whose closure is mapped homeomorphically onto $\bar{F}$ by $f^{n}$. Moreover, $f^{k}(z) \rightarrow \infty$ as $z \rightarrow \infty$ in $\tau$, for all $k \geq n+1$.

Definition 3.4 (Fundamental tails). Let $n \geq 1$. A connected component $\tau$ of $f^{-n}\left(W_{0}\right)$ is called a fundamental tail of level $n$. In particular, the fundamental tails of level 1 are precisely the fundamental domains of $f$.

Proposition 3.5 (Facts about fundamental tails). Fundamental tails of the same level are disjoint. Moreover, for every $N$ and any compact set $K$, there are at most finitely many fundamental tails of level at most $N$ that meet $K$.

If $\tau$ is a fundamental tail of level $n>1$, then $f(\tau)$ is a fundamental tail of level $n-1$.

Proof. Recall that the fundamental tails of level $N$ are precisely the connected components of $f^{-N}\left(W_{0}\right)$. Hence they are pairwise disjoint, and the second claim follows from Lemma 2.1. The final claim holds by definition.

LEMMA 3.6 (Fundamental domain associated to fundamental tail). Let $\tau$ be a fundamental tail of level $n$. Then there is a unique fundamental domain $F$ such that $F \cap \tau$ is unbounded. In fact, if $n>1$, then $F$ contains all sufficiently large points of $\bar{\tau}$.

Proof. We proceed by induction. The claim is trivial for $n=1$. Now suppose that $n>1$. By induction, there is a unique fundamental domain $F_{1}$ whose intersection with the fundamental tail $f(\tau)$, of level $n-1$, is unbounded.

Let $A_{1}$ be the unbounded connected component of $\overline{F_{1}} \backslash D$, and let $A_{2}$ be the unbounded connected component of $A_{1} \cap \overline{f(\tau)} \backslash \bar{D}$. Then $f(\tau) \backslash A_{2}$ is bounded. Indeed, this is clear if $n=2$, and otherwise follows from the inductive hypothesis.

Recall that $f: \bar{\tau} \rightarrow \overline{f(\tau)}$ is a homeomorphism. Since $A_{2} \subset W_{0}$, there is a unique fundamental domain $F$ containing $A:=f^{-1}\left(A_{2}\right) \cap \bar{\tau}$, and $\bar{\tau} \backslash A \subset \bar{\tau} \backslash F$ is bounded, as claimed.

It follows from the above that we can associate natural symbolic sequences to fundamental tails.

Definition 3.7 (Addresses of fundamental tails). Let $\tau$ be a fundamental tail of level $n$, and denote by $F_{k}(\tau)$ the unique fundamental domain whose intersection with the fundamental tail $f^{k}(\tau)$ is unbounded. We will call the finite sequence $\underline{s}=$ $F_{0}(\tau) F_{1}(\tau) \ldots F_{n-1}(\tau)$, of length $n$, the (finite) external address of $\tau$.

Conversely, we can construct a fundamental tail having an arbitrary prescribed (finite) address by taking repeated pull-backs along the correct branches. Recall that a sequence $\underline{s}^{1}$, say of length $n$, is a prefix of another sequence $\underline{s}^{2}$ of length $m \geq n$ if the first $n$ entries of $\underline{s}^{2}$ coincide with those of $\underline{s}^{1}$. 
Definition And Lemma 3.8 (Tails at a given address). Let $\underline{s}=F_{0} F_{1} \ldots$ be a finite or infinite sequence of fundamental domains having length at least $n \geq 1$. Then there is a unique fundamental tail $\tau$ of level $n$ having address $\underline{\tilde{s}}:=F_{0} F_{1} \ldots F_{n-1}$. We denote this fundamental tail by $\tau_{n}(\underline{s})$. We also define the inverse branches

$$
f_{\underline{s}}^{-n}:=\left(\left.f^{n}\right|_{\tau_{n}(\underline{s})}\right)^{-1}: W_{0} \rightarrow \tau_{n}(\underline{s}) .
$$

Proof. The proof is by induction on the level $n$ of the tails. For $n=1$ and for every fundamental domain $F_{0}$, the fundamental tail of level 1 and address $F_{0}$ is the fundamental domain $F_{0}$ itself, which is unique and has address $F_{0}$ by definition. Now let $\underline{s}$ be in the claim and let $\tau=\tau_{n-1}(\sigma(\underline{s}))$ be the fundamental tail of level $n-1$ and address $F_{1} \ldots F_{n-1}$. This tail exists and is unique by the inductive hypothesis. Let $R$ be sufficiently large, and let $\tau^{1}$ be the unique unbounded connected component of $\tau \backslash \overline{D_{R}(0)}$. By Lemma 3.6, $\tau^{1}$ is contained in the fundamental domain $F_{1}=F_{0}(\tau)$ for sufficiently large $R$. Hence, if we additionally assume that $R$ is greater than the radius of $D$, we have $\tau^{1} \subset f\left(F_{0}\right)=W_{0}$. So there is a unique connected component $\tilde{\tau}^{1}$ of $f^{-1}\left(\tau^{1}\right)$ contained in $F_{0}$, and a unique connected component $\tilde{\tau}$ of $f^{-1}(\tau)$ containing $\tilde{\tau}^{1}$. Then $F_{0}(\tilde{\tau})=F_{0}$; i.e., $F_{0}$ is the initial entry in the address of the fundamental tail $\tilde{\tau}$, which hence has address $\underline{s}$.

The following are immediate consequences of the preceding results and definitions.

Observation 3.9. Let $\tau$ be a fundamental tail of level $n$, and let $\underline{s}$ be the address of $\tau$. Then the address of $f(\tau)$ is $\sigma(\underline{s})$, where $\sigma$ denotes the shift map.

Suppose that $\tau^{1}$ and $\tau^{2}$ are fundamental tails of levels $n_{1}$ and $n_{2}$, with $n_{1} \geq n_{2}$. Let $\underline{s}^{1}$ and $s^{2}$ be the addresses of $\tau^{1}$ and $\tau^{2}$, respectively. Then $\tau^{1} \cap \tau^{2}$ is unbounded if and only if $\underline{s}^{1}$ is a prefix of $\underline{s}^{2}$. In this case, if addditionally $n_{1}>n_{2}$, all sufficiently large points of $\overline{\tau^{1}}$ lie in $\tau^{2}$.

\section{Dreadlocks}

Maintaining the same notation as in the previous section, we now define and study the central objects of this article: dreadlocks. Recall that $\mathcal{P}(f)$ is bounded; the main goal of this section is to show that, under this assumption, each of the sets $J_{s}^{0}$ defined in Section 2 can be consistently extended to a larger - and, in a certain sense, maximal - set $J_{\underline{s}}$. The intersection of $J_{\underline{s}}$ with the escaping set $I(f)$ forms the dreadlock $G_{\underline{s}}$ at address $\underline{s}$.

As we shall see, each dreadlock is an unbounded connected set of escaping points, the escaping set can be written as the union of dreadlocks, and via their external addresses the collection of dreadlocks is endowed with a natural combinatorial structure. Furthermore, the definition of dreadlocks does not depend on the initial choices made in the construction of fundamental domains. Together these facts indicate that dreadlocks can indeed be considered a natural generalisation of "hairs" or "rays". 
For a fundamental domain $F$, recall that $\stackrel{\infty}{F}$ denotes the unbounded connected component of $F \backslash \bar{D}$. We extend this definition to fundamental tails as follows.

DEFINITION 4.1 (Unbounded parts of tails). Let $\tau$ be a fundamental tail of level $n$. We define $\stackrel{\infty}{\tau}$ to be the unbounded connected component of $\tau \backslash f^{-(n-1)}(\bar{D})$. In other words, if $F=f^{n-1}(\tau)$, then $\stackrel{\infty}{\tau}$ is the component of $f^{-(n-1)}(\stackrel{\infty}{F})$ contained in $\tau$.

Observe that, if $\underline{s}$ is an external address and $n \geq 2$, then $\stackrel{\infty}{\tau}_{n}(\underline{s})$ is precisely the unbounded connected component of $\tau_{n}(\underline{s}) \cap \tau_{n-1}(\underline{s})$.

Definition 4.2 (Dreadlocks). Let $\underline{s}$ be an (infinite) external address. We say that a point $z \in \mathbb{C}$ has external address $\underline{s}$ if $z \in \stackrel{\infty}{\tau}_{n+1}(\underline{s})$ for all sufficiently large $n$.

We denote the set of all points $z \in \mathbb{C}$ having external address $\underline{s}$ by $J_{\underline{s}}$. The dreadlock $G_{\underline{s}}$ is defined to be $G_{\underline{s}}:=J_{\underline{s}} \cap I(f)$.

REMARK. If $z \in J_{\underline{s}}$, then $f^{n}(z)$ belongs to $\stackrel{\infty}{F}_{n}$ for all sufficiently large $n$. In particular, $z \in J(f)$ by Lemma 2.6. Observe, however, that $J_{\underline{s}}$ is not closed in general.

Note that other notions of points having external address $\underline{s}$ appear in the literature; for example, in [Rem07a], $z$ is said to have address $\underline{s}=F_{0} F_{1} \ldots$ if $f^{n}(z) \in F_{n}$ for all $n \geq 0$. The advantage of the above definition, in the context of postsingularly bounded functions, is that we shall see that every escaping point has an external address (Corollary 4.5), and that this address is in a certain sense independent of the initial choice of fundamental domains (Observation 4.12).

Lemma 4.3 (Properties of addresses and dreadlocks). Suppose that $\underline{s}$ is an external address and $z \in J_{\underline{s}}$. Then

(a) $z \notin J_{\underline{\tilde{s}}}$ for $\underline{s} \neq \underline{\underline{s}}$.

(b) The point $f(z)$ has address $\sigma(\underline{s})$.

(c) If $w \in f^{-1}(z)$, then $w$ has an address in $\sigma^{-1}(\underline{s})$, and every such address is realised by exactly one element of $f^{-1}(z)$.

(d) The restriction $f: J_{\underline{s}} \rightarrow J_{\sigma(\underline{s})}$ is a continuous bijection.

Proof. The first claim is trivial since fundamental tails of a given level are disjoint. Now write $\underline{s}=F_{0} F_{1} F_{2} \ldots$; observe that $f\left(\stackrel{\infty}{\tau}_{n+1}(\underline{s})\right)=\stackrel{\infty}{\tau}_{n}(\sigma(\underline{s}))$ and that $f: \stackrel{\infty}{\tau}_{n+1}(\underline{s}) \rightarrow \stackrel{\infty}{\tau}_{n}(\sigma(\underline{s}))$ is a conformal isomorphism.

By definition, $z$ belongs to $\stackrel{\infty}{\tau}_{n}(\underline{s})$ for all sufficiently large $n$; say for $n \geq n_{0}$. Let $n \geq \max \left(1, n_{0}-1\right)$. Then $f(z) \in f\left(\left(_{\tau}^{\infty}{ }_{n+1}(\underline{s})\right)=\stackrel{\infty}{\tau}_{n}(\sigma(\underline{s}))\right.$, as claimed in (b).

Now let $w \in f^{-1}(z)$. For $n \geq n_{0}$, we have $z=f(w) \in \stackrel{\infty}{n}_{n}(\underline{s})$. In particular, for $n \geq n_{0}+1$, there is a fundamental tail $\vartheta_{n}$ such that $f\left(\vartheta_{n}\right)=\tau_{n-1}(\underline{s})$ and $w \in \stackrel{\infty}{\vartheta}_{n}$. Recall that $\stackrel{\infty}{\tau}_{n}(\underline{s}) \subset \tau_{n-1}(\underline{s})$, and that the intersection $\stackrel{\infty}{\vartheta}_{n+1} \cap \vartheta_{n}$ is nonempty (since it contains $w$ ). Hence $\vartheta_{n+1} \subset \vartheta_{n}$ for all $n \geq n_{0}+1$. That is, $\vartheta_{n+1}$ tends to infinity within $\vartheta_{n}$. So if $F$ is the fundamental domain whose intersection with $\vartheta_{n_{0}+1}$ is unbounded, then $\vartheta_{n}$ tends to infinity in $F$ for $n \geq n_{0}+1$. It follows that $\vartheta_{n}=\tau_{n}(F \underline{s})$. In particular, $w$ has address $F \underline{s} \in \sigma^{-1}(\underline{s})$. 
Conversely, let $\underline{\tilde{s}} \in \sigma^{-1}(\underline{s})$; that is, $\underline{\tilde{s}}=F \underline{s}$ for some fundamental domain $F$. Then the fundamental tail $\vartheta_{n_{0}+1}$ of level $n_{0}+1$ associated to the address $\underline{\tilde{s}}:=F \underline{s}$ is a component of $f^{-1}\left(\tau_{n_{0}}\right)$. Hence there is a unique element $w$ of $f^{-1}(z)$ in $\vartheta_{n_{0}+1}$, and $w$ has address $\underline{\tilde{s}}$ as above.

The final claim follows from the previous two.

The following proposition establishes a connection between the sets $J_{\underline{s}}$ defined in Definition 4.2 and the sets $J_{\underline{s}}^{0}$ studied in Section 2.

Proposition $4.4\left(J_{\underline{s}}\right.$ and $\left.J_{\underline{s}}^{0}\right)$. Let $\underline{s}$ be an (infinite) external address. If $z \in J_{\underline{s}}^{0}$, then $z \in \stackrel{\infty}{\tau}_{n}(\underline{s})$ for all $n \geq 1$. In particular, $J_{\underline{s}}^{0} \subset J_{\underline{s}}$.

Proof. This is essentially the content of the second paragraph of the proof of [Rem07a, Theorem 1.1]. Since that proof is somewhat concise, and we are using a different terminology, we provide the details.

For $n \geq 1$, let $\vartheta_{n}$ denote the fundamental tail of level $n$ containing $z$. By assumption, $f^{n-1}(z) \in \stackrel{\infty}{F}_{n-1}$, and hence $z \in \stackrel{\infty}{\vartheta}_{n}$.

We claim that $\stackrel{\infty}{\vartheta+1}_{n+1} \subset \vartheta_{n}$; that is, $\vartheta_{n+1}$ tends to infinity within $\vartheta_{n}$. Indeed, $\stackrel{\wp}{n+1}_{n}$ is a connected component of $f^{-n}\left(F_{n}\right)$ and $\vartheta_{n}$ is a connected component of $f^{-n}\left(W_{0}\right)$. Since $\stackrel{\infty}{F}_{n} \subset W_{0}$ and $\stackrel{\infty}{\vartheta}_{n+1} \cap \vartheta_{n} \neq \emptyset$, the claim follows.

Inductively, $\vartheta_{n}$ tends to infinity within $F_{0}=\vartheta_{1}$ for each $n \geq 0$. Applying this fact to $f^{k}(z)$, we see likewise that $f^{k}\left(\vartheta_{n}\right)$ tends to infinity within $\bar{F}_{k}$ for $n \geq k$. Thus, for any $n \geq 1$, we conclude that $\vartheta_{n}=\tau_{n}(\underline{s})$, and hence $z \in \stackrel{\infty}{\vartheta}_{n}=\stackrel{\infty}{\tau}_{n}(\underline{s})$, as desired.

COROLlary 4.5 (Escaping points are organised in dreadlocks).

(a) A point $z$ has an external address if and only if there is $n$ and an external address $\underline{s}$ such that $f^{n}(z) \in J_{\sigma^{n}(\underline{s})}^{0}$.

(b) There is a number $R>0$ with the following property. If $z \in J(f)$ is such that $\left|f^{n}(z)\right| \geq R$ for all sufficiently large $n$, then $z$ has an external address $\underline{s}$.

(c) Every escaping point $z \in I(f)$ has an external address $\underline{s}$, and hence belongs to a dreadlock $G_{\underline{s}}$.

Proof. The "only if" direction of (a) is immediate from Definition 4.2. On the other hand, if $z$ has the stated property, then $f^{n}(z)$ has address $\underline{s}$ by Proposition 4.4 , and hence $z$ also has an external address by Lemma 4.3.

To prove (b), let $\mathcal{F}$ be the set of fundamental domains that intersect $D$. Recall that $F \backslash \stackrel{\infty}{F}$ is bounded for all $F \in \mathcal{F}$, and that $\mathcal{F}$ is finite by Lemma 2.1. Now fix

$$
R>\sup \left\{|z|: z \in \bar{D} \cup \bigcup_{F \in \mathcal{F}} F \backslash \stackrel{\infty}{F}\right\} \text {. }
$$

Suppose that $\left|f^{n}(z)\right| \geq R$ for all $n \geq n_{0}$. Then, for all $n \geq n_{0}, f^{n}(z)$ belongs to some fundamental domain $F$, and by choice of $R$, it must belong to $\stackrel{\infty}{F}$. Now $z$ has an address by (a).

Finally, (c) is an immediate consequence of (b). 
REMARK 4.6 Under the hypotheses of Proposition $4.4, f^{n}(z)$ belongs to $\stackrel{\infty}{F}_{n}$ for all $n$. However, it may well be that $z$ belongs to a bounded component of $\tau_{k}(\underline{s}) \backslash \bar{D}$ for all sufficiently large $k$ and similarly for all points on the forward orbit of $z$.

Indeed, typically when $I(f)$ does not consist of hairs, exactly this is the case for many escaping points, so Proposition 4.4 is not at all trivial, and uses the boundedness of the postsingular set in an essential manner. No analogue thereof is currently known for functions with unbounded postsingular set, and in particular in this setting there is no canonical way of associating external addresses to arbitrary escaping points as in Corollary 4.5. This is a major challenge in showing the unboundedness of components of the escaping set. (Compare Corollary 4.11 below.)

Connectedness of dreadlocks and uniform escape to infinity. Thanks to Proposition 4.4, we can use the results of Section 2 to study dreadlocks. We use the following definition from [Rem16].

Definition 4.7 (Escaping composants). Let $z \in I(f)$. Then the escaping composant $\mu(z)=\mu(z, f)$ is the union of all connected sets $A$ containing $z$ on which the iterates of $f$ tend to infinity uniformly.

REMARK. While $\mu(z)$ is a union of sets on which the iterates tend to infinity uniformly, typically $f^{n}$ will not tend to infinity uniformly on $\mu(z)$.

LEMMA 4.8 (Dreadlocks and uniform escape). Let $\underline{s}$ be an external address, and suppose that $z \in G_{\underline{s}}$. Let $A \ni z$ be a connected set and suppose that there is $N \geq 0$ such that $f^{n}(A) \cap \bar{D}=\emptyset$ for $n \geq N$. (In particular, this is the case when the iterates of $f$ tend to infinity uniformly on $A$.)

Then there is $n_{0} \geq N$ such that $A \subset \stackrel{\infty}{\tau}_{n}(\underline{s})$ for $n \geq n_{0}$. In particular, $\mu(z) \subset G_{\underline{s}}$. Proof Let $n_{0} \geq N+1$ be such that $z \in \stackrel{\infty}{\tau}_{n}(\underline{s})$ for $n \geq n_{0}$. Let $n \geq n_{0}$. Then

$$
A \subset f^{-n}\left(W_{0}\right) \cap f^{-(n-1)}\left(W_{0}\right) .
$$

Now $\stackrel{\infty}{\tau}_{n}(\underline{s})$ is a connected component of the set on the right-hand side, $z \in A \cap \stackrel{\infty}{\tau}_{n}(\underline{s})$, and $A$ is connected. Hence $A \subset \stackrel{\infty}{\tau}_{n}(\underline{s})$ for $n \geq n_{0}$, and all points in $A$ have address $\underline{s}$.

We remark that a dreadlock $G_{\underline{s}}$ may contain uncountably many different escaping composants. Moreover, it is possible for $\mu(z)$ to consist of a single point. (See [Rem16, Theorem 1.6].) However, by the results of Section 2, $G_{\underline{s}}$ contains a distinguished uniformly escaping composant, namely the one consisting of those points for which the set $A$ can be taken to be unbounded.

Definition AND Lemma 4.9 (The core of a dreadlock). Let $\underline{s}$ be an infinite external address. Let $\mathcal{X}$ be the collection of all closed, unbounded, connected sets $X \subset G_{\underline{s}}$ on which the iterates of $f$ tend to infinity uniformly. Then no element of $\mathcal{X}$ separates the plane. Furthermore, $\mathcal{X}$ is linearly ordered by inclusion; i.e., if $X_{1}, X_{2} \in \mathcal{X}$ then $X_{1} \subset X_{2}$ or $X_{2} \subset X_{1}$. 
In particular, if $J_{\underline{s}} \neq \emptyset$, then the union $\mu_{\underline{s}}:=\bigcup \mathcal{X} \subset G_{\underline{s}} \neq \emptyset$ satisfies $\mu_{\underline{s}}=\mu(z)$ for all $z \in \mu_{\underline{s}}$. We call $\mu_{\underline{s}}$ the core of the dreadlock $G_{\underline{s}}$.

Proof If $X \in \mathcal{X}$, then there is $n_{0} \geq 1$ such that $X \subset \stackrel{\infty}{\tau}_{n}(\underline{s})$ for $n \geq n_{0}$. In particular, $f^{n}(X) \subset J_{\sigma^{n}(\underline{s})}^{0}$ for $n \geq n_{0}$.

Let $n \geq n_{0}$. Since $f^{n}: \tau_{n}(\underline{s}) \rightarrow W_{0}$ is a conformal isomorphism, and $X$ is unbounded, it follows that $f^{n}(X)$ is unbounded for all $n$. By Lemma 2.6 we know that $f^{n}(X)$, and hence $X$, does not separate the plane.

Furthermore, if $X_{1}, X_{2} \in \mathcal{X}$, then we can choose $n_{0}$ sufficiently large so that the above holds for both sets. It follows from part (b) of Theorem 2.5 that one of $f^{n_{0}}\left(X_{1}\right)$ and $f^{n_{0}}\left(X_{2}\right)$ is contained in the other, and the same holds for $X_{1}$ and $X_{2}$.

By part (a) of Theorem 2.5, together with Proposition 4.4, the set $\mu_{\underline{s}}$ (as defined in the statement of the lemma) is non-empty. By the fact we just proved, $\mu_{\underline{s}}$ is a nested union of connected sets, and hence itself connected. Finally suppose that $z \in \mu_{\underline{s}}$; so $z \in X_{z}$ for some $X_{z} \in \mathcal{X}$. Since $\mathcal{X}$ is linearly ordered by inclusion, we have

$$
\mu_{\underline{s}}=\bigcup\left\{X \in \mathcal{X}: X_{z} \subset X\right\} \subset \mu(z) .
$$

Conversely, if the iterates of $f$ tend to infinity uniformly on the connected set $A \ni z$, then they do so also on the closed, unbounded and connected set $\bar{A} \cup X_{z}$, which is contained in $G_{\underline{s}}$ by Lemma 4.8. Hence $A \subset \mu_{\underline{s}}$, and we have proved $\mu(z)=\mu_{\underline{s}}$, as desired.

Proposition 4.10 (Dreadlocks are connected). Let $\underline{s}$ be an external address with $J_{\underline{s}} \neq \emptyset$. Then $\mu_{\underline{s}}$ is dense in $J_{\underline{s}}$ and $G_{\underline{s}}$. In particular, both of these sets are connected and unbounded.

Proof Let $z \in J_{\underline{s}}$. By Corollary 4.5, there is $n_{0}$ such that $f^{n}(z) \in J_{\sigma^{n}(\underline{s})}^{0}$ for $n \geq n_{0}$. Let $X_{n}$ be the unbounded connected subset of $\mu_{\sigma^{n}(\underline{s})}$ whose existence is guaranteed by Theorem 2.5. Recall that $X_{n}$ can be connected to $f^{n}(z)$ by a curve $\gamma_{n} \subset \mathbb{C} \backslash \bar{D}$ of cylindrical length at most $4 \pi$ such that $\gamma_{n}$ is homotopic to a curve in $W_{0}$. In particular, the pullback $\tilde{\gamma}_{n}$ of $\gamma_{n}$ along the orbit of $z$ is a curve connecting $z$ to the set $\tilde{X}_{n}:=f_{\underline{s}}^{-n}\left(X_{n}\right) \subset \mu(\underline{s})$.

The density $\rho_{\Omega}$ of the hyperbolic metric on $\Omega$ tends to zero like $1 /|z||\log z|$. (Recall that $\Omega$ is the unbounded connected component of $\mathbb{C} \backslash \mathcal{P}(f)$.) Therefore the hyperbolic length $\ell_{\Omega}\left(\gamma_{n}\right)$ is also uniformly bounded, independently of $n$. It follows by Proposition 3.1 that $\ell_{\Omega}\left(\tilde{\gamma}_{n}\right) \rightarrow 0$ as $n \rightarrow \infty$. Hence $\operatorname{dist}_{\Omega}\left(z, \tilde{X}_{n}\right) \rightarrow 0$, and $z \in \overline{\mu_{\underline{s}}}$, as claimed.

Recall that the (relative) closure of a connected set is again connected. So $J_{s}$ and $G_{\underline{s}}$ are connected and unbounded, as the dense subset $\mu_{\underline{s}}$ has these properties.

We note that the above result (and its proof) is essentially a reformulation of the main argument in the proof of the main theorem of [Rem07a], which we recover as follows. 
Corollary 4.11 (Unbounded sets of escaping points). If $f$ is a postsingularly bounded entire function, then every connected component of the escaping set $I(f)$ is unbounded.

Proof Let $z \in I(f)$. By Corollary 4.5, $z \in G_{\underline{s}}$ for some external address $\underline{s}$, and by Proposition 4.10, $G_{\underline{s}}$ is an unbounded connected subset of $I(f)$.

Independence of dreadlocks from the construction. Note that the definition of addresses, and hence of dreadlocks, depends a priori on the choice of fundamental domains, and hence on the domain $W_{0}$ (i.e., on the choice of the disc $D$ and the curve $\delta$ ). However, if dreadlocks are to be considered canonical objects associated to $f$, then this dependence should not be essential. We briefly discuss why this is indeed the case.

Suppose that $\hat{W}_{0}$ is a different choice of base domain, and that $\hat{\tau}$ is a fundamental tail of level at least 2 , using this alternative initial configuration.

Then it is easy to see that there is a unique fundamental domain $F$ for the original domain $W_{0}$ such that all sufficiently large points of $\hat{\tau}$ lie in $F$. Indeed, if $z \in \hat{\tau}$ is sufficiently large, then $f(z), f^{2}(z) \notin \bar{D}$, and in particular $f(z) \notin \delta$. So $f(z) \in W_{0}$, and the claim is established.

In particular, if $\underline{\hat{s}}$ is an external address with respect to $\hat{W}_{0}$, then we can associate to $\underline{\hat{s}}$ an address $\underline{s}=F_{0} F_{1} \ldots$ with respect to $W_{0}$. Here for each $n \geq 0, F_{n}$ is the fundamental domain associated, in the above manner, to the fundamental tails $\hat{\tau}_{k}\left(\sigma^{n}(\underline{\hat{s}})\right.$ ), for $k \geq 2$. (Observe that $F_{n}$ is independent of $k$.) It is easy to see that, in turn, $\underline{\hat{s}}$ can be obtained from $\underline{s}$ using the same procedure in the opposite direction, so that the correspondence $\underline{s} \mapsto \underline{\hat{s}}$ is a bijection between external addresses defined using the original configuration $W_{0}$, and those defined using the modified one. The following observation is an easy consequence of this construction.

Observation 4.12 (Dreadlocks are independent of the initial configuration). Let $\underline{s}$ and $\underline{\hat{s}}$ correspond to each other as in the above construction; let $G_{\underline{s}}$ be the dreadlock obtained by using preimages of $W_{0}$ in the construction, and let $\hat{G}_{\underline{\hat{s}}}$ be the corresponding dreadlock according to the choice $\hat{W}_{0}$. Then $G_{\underline{s}}=\hat{G}_{\underline{\hat{s}}}$.

In other words, the collection $\mathcal{G}=\left\{G_{\underline{s}}\right\}$ of subsets of $J(f)$ is independent of the initial choice of $W_{0}$.

We remark that the same is not true for the set $J_{\underline{s}}$. Indeed, suppose that $z$ is a non-escaping point that has an external address for one choice of $W_{0}$. Then we can choose a different initial configuration $\tilde{W}_{0}$, where the initial disc $\tilde{D}$ is chosen sufficiently large to ensure that $z$ enters $\tilde{D}$ infinitely many times. Clearly $z$ does not have an external address with respect to this configuration.

In similar fashion, we see that dreadlocks are preserved under iteration. Indeed, let $n \geq 1$. Suppose that $\hat{D}$ is a disc centred around zero such that $f^{n-1}(D) \subset \hat{D}$, and that $\hat{\delta} \subset \delta$ connects $\hat{D}$ to $\infty$ outside of $\hat{D}$. Then $\hat{W}_{0}:=\mathbb{C} \backslash(\operatorname{cl}(\hat{D}) \cup \hat{\delta})$ is a valid initial configuration for $f^{n}$. Every fundamental domain of $f^{n}$ (with respect to 
$\left.\hat{W}_{0}\right)$ is contained in a unique fundamental tail of level $n$ for $f$. Conversely, every fundamental tail of $f$ contains exactly one fundamental domain of $f^{n}$. Hence there is a natural bijection between the fundamental domains of $f^{n}$ and finite external addresses of length $n$ for $f$, and thus between external addresses of $f^{n}$ and those of $f$. In particular, we obtain the following.

Observation 4.13 (Dreadlocks and iterates). Every dreadlock of $f$ is a dreadlock of $f^{n}$, for $n \geq 1$, and vice versa.

Cyclic order of addresses and dreadlocks. There is a natural cyclic order on the set of fundamental tails of any given level, and in particular on the set of fundamental domains of $f$ : if $A, B, C$ are fundamental tails, then $A \prec B \prec C$ means that $B$ tends to infinity between $A$ and $C$ in positive orientation. (See Section 13 for background on the cyclic order near infinity.) We can also define a cyclic order on the collection of dreadlocks, by choosing for each a closed, connected, unbounded set on which the iterates tend to infinity, as in Proposition 4.10, and considering the cyclic order of these sets.

Recall that the function $f$ acts in a natural way on dreadlocks, and maps fundamental tails of level $n+1$ to fundamental tails of level $n$. By the remark above, this action locally preserves cyclic order, in the following sense. Let $A \prec B \prec C$ be either dreadlocks or fundamental tails of some fixed level $n>1$. If the addresses of $A, B$ and $C$ all have the same initial entry, then $f(A) \prec f(B) \prec f(C)$.

We can also define a "lexicographical" cyclic order on (finite or infinite) external addresses. To do so, we use the curve $\delta$ to convert the cylic order on fundamental domains to a linear order in the usual sense, setting $F<\tilde{F}$ if and only if $\delta \prec F \prec \tilde{F}$. This linear order gives rise to a lexicographic order $<$ on external addresses in the usual sense. The cyclic order on addresses is then defined by $\underline{s}^{1} \prec \underline{s}^{2} \prec \underline{s}^{3}$ if and only if $s^{1}<s^{2}<s^{3}, s^{2}<s^{3}<s^{1}$, or $s^{3}<s^{1}<s^{2}$.

It follows from what was written above that this cyclic order on addresses agrees with the cyclic order of the associated fundamental tails or dreadlocks.

Disjoint-type addresses. To conclude this section, we discuss a particularly wellbehaved type of dreadlock. Note that, in general, the points in the closure of a dreadlock $G_{\underline{\underline{s}}}$ need not belong to $J_{\underline{s}}$. Indeed, this is the case for those dreadlocks of greatest interest to us, namely those accumulating on a periodic point whose orbit does not lie outside of $\bar{D}$. We show now that this cannot occur when the address $\underline{s}$ (or a forward iterate thereof) contains only fundamental domains that do not intersect $\bar{D}$.

LEMma 4.14 ((Eventually) disjoint-type addresses). Let $\underline{s}=F_{0} F_{1} \ldots$ be an external address, and suppose that there is $n_{0} \geq 0$ such that $\overline{F_{n}}$ does not intersect $\partial D$ for $n \geq n_{0}$. Then $\overline{G_{\underline{s}}}=J_{\underline{s}}=f_{\underline{s}}^{-n}\left(J_{\sigma^{n}(\underline{s})}^{0}\right) \subset \tau_{n}(\underline{s})$ for $n \geq n_{0}$.

Proof By assumption, we have $\overline{F_{n}} \subset W_{0}$, and in particular $\stackrel{\infty}{F}_{n}=F_{n}$, for $n \geq n_{0}$. Thus $\overline{\tau_{n+1}(\underline{s})} \subset \tau_{n}(\underline{s})$ for $n \geq n_{0}$ (where we use the convention that $\tau_{0}(\underline{s})=W_{0}$ for 
convenience). In particular, $\stackrel{\infty}{\tau}_{n+1}(\underline{s})=\tau_{n+1}(\underline{s})$, and

$$
J_{\underline{s}}=\bigcup_{k>0} \bigcap_{j \geq k}{\mathcal{\tau}_{j}}_{j}(\underline{s})=\bigcap_{j>n} \tau_{j}(\underline{s})=\left\{z \in \tau_{n}(\underline{s}): f^{j}(z) \in F_{j} \text { for } j \geq n\right\}=f_{\underline{s}}^{-n}\left(J_{\sigma^{n}(\underline{s})}^{0}\right)
$$

by definition. Furthermore,

$$
\overline{G_{\underline{s}}}=\overline{J_{\underline{s}}}=\bigcap_{n>n_{0}} \overline{\tau_{n}(\underline{s})} \subset \bigcap_{n>n_{0}} \tau_{n+1}(\underline{s})=J_{\underline{s}}
$$

(where the first equality holds by Proposition 4.10). This completes the proof.

REMARK 4.15 (Disjoint-type addresses). If $n_{0}=0$, then we say that $\underline{s}$ is of disjoint type (since $\tau_{n}(\underline{s})$ and $\tau_{n^{\prime}}(\underline{s})$ have disjoint boundaries for $n \neq n^{\prime}$ ). For a disjoint-type function (i.e., one that is hyperbolic with connected Fatou set), all addresses are of disjoint-type, given a suitable choice of fundamental domains. In this case, every component of the Julia set $J(f)$ is one of the sets $J_{\underline{s}}=J_{\underline{s}}^{0}$, and the closure $J_{\underline{s}} \cup\{\infty\}$ in $\hat{\mathbb{C}}$ is called a Julia continuum. Compare [Rem16]. Suppose that, additionally, $G_{\underline{s}}$ is a hair (in the sense of Definition 5.1 below). Then it follows, using [Rem16, Corollary 5.6], that either $G_{\underline{s}}=J_{\underline{s}}$, and $J_{\underline{s}}$ is an arc to $\infty$ on which the iterates of $f$ tend to infinity uniformly, or $J_{\underline{s}} \backslash G_{\underline{s}}$ contains a single non-escaping point $z_{0}$; here $G_{\underline{s}}$ is an arc connecting $z_{0}$ to $\infty$. (For functions satisfying a head-start condition, this follows already from the results of [RRRS11], without the need for the results of [Rem16].)

In fact, it can be shown that, whenever $\underline{s}$ is of disjoint type, the set $J_{\underline{s}}$ is homeomorphic to the component of the Julia set of a suitable disjoint-type function, with escaping points corresponding to escaping points. (Compare also Theorem 7.2 below.) Hence the above observation remains true for disjoint-type addresses, even if $f$ itself is not of disjoint type. So we may think of dreadlocks at disjoint-type addresses as always "landing". When $\underline{s}$ is bounded, we show below that this is true in a precise sense (see Proposition $6.5(\mathrm{~d})$ ).

However, when $G_{\underline{s}}$ is not a hair, it may happen that $J_{\underline{s}}$ contains a dense or uncountable set of non-escaping points, even when $f$ and hence all addresses are of disjoint type [Rem16, Theorem 2.3]. In this article, we only consider landing properties for dreadlocks at bounded external addresses, so these subtleties will not become relevant.

\section{Hairs and Dreadlocks}

We shall now discuss the relationship between hairs and dreadlocks. The term "hair" was coined by Devaney in the 1980s (see [Dev84, p. 168]), and is commonly used in an informal manner to refer to dynamically natural curves in Julia sets of transcendental entire functions. We use the following convention. (See Remark 5.6 below for comparison with some other definitions in the literature.) 
Definition 5.1 (Hairs). A dreadlock $G_{\underline{s}}$ is a hair if one of the following holds.

(a) There is a homeomorphism $\gamma:[0, \infty] \rightarrow G_{\underline{s}} \cup\{\infty\}$ such that $\gamma(\infty)=\infty$ and $\left.f^{n}\right|_{G_{\underline{\underline{s}}}} \rightarrow \infty$ uniformly.

(b) There is a continuous bijection $\gamma:(0, \infty] \rightarrow G_{\underline{s}} \cup\{\infty\}$ such that $\gamma(\infty)=\infty$ and $\left.f^{n}\right|_{\gamma\left(\left[t_{0}, \infty\right)\right)} \rightarrow \infty$ uniformly, for any $t>0$.

REMARK 1. In general, the bijection in (b) is not a homeomorphism. Indeed, even for exponential maps a hair may accumulate upon itself. Compare e.g. [DJ02, DJR05, Rem07b, Wor18].

REMARK 2. The second part of condition (a) is essential. Indeed, by [Rem16, Theorem 2.10], there exists a postsingularly bounded entire function having a dreadlock which is an arc connecting a finite endpoint to infinity, but such that the iterates on this arc do not tend to infinity uniformly.

On the other hand, the second part of condition (b) can be shown to be inessential. That is, suppose $\gamma:(0, \infty] \rightarrow G_{\underline{s}} \cup\{\infty\}$ is a continuous bijection with $\gamma(\infty)=\infty$. Then, possibly after reversing the orientation of $\gamma$ on $(0, \infty)$, for all $t>0$ we have $\left.f^{n}\right|_{\gamma\left(\left[t_{0}, \infty\right)\right)} \rightarrow \infty$ uniformly. We shall not provide the (somewhat lengthy) proof, as we do not require this fact in our paper.

Observation 5.2 (Properties of hairs).

(a) $G_{\underline{s}}$ is a hair if and only if $G_{\sigma(\underline{s})}$ is a hair. Up to suitable reparameterisation, the corresponding functions $\gamma$ and $\tilde{\gamma}$ satisfy $\tilde{\gamma}(t)=f(\gamma(t))$.

(b) If $\underline{s}$ is a bounded external address, then $\left.f^{n}\right|_{G_{\underline{s}}}$ does not tend to infinity uniformly. In particular, if $\underline{s}$ is bounded, then $G_{\underline{s}}$ is a hair if and only if condition (b) of Definition 5.1 holds.

Proof To prove the first claim, note that $f: G_{\underline{s}} \rightarrow G_{\sigma(\underline{s})}$ is a continuous bijection. Furthermore, for every $n \geq 2,\left.f\right|_{\tau_{n}(\underline{s})}$ extends to a homeomorphism $\tau_{n}(\underline{s}) \cup\{\infty\} \rightarrow$ $\tau_{n-1}(\sigma(\underline{s})) \cup\{\infty\}$. If $G_{\underline{s}}$ is a hair, define $\tilde{\gamma}$ as in the claim. Clearly it is only necessary to show that $\tilde{\gamma}(t) \rightarrow \infty$ as $t \rightarrow \infty$, which follows from the above fact since $\left.\gamma\right|_{[1 / 2,1)} \subset \tau_{n}$ for sufficiently large $n$. For the converse direction, we define $\gamma(t):=\left(\left.f\right|_{\tau_{n}(\underline{s})}\right)^{-1}(\tilde{\gamma}(t))$, where $n$ is sufficiently large depending on $t$, and proceed analogously.

Now suppose that $\underline{s}$ is bounded, and let $R>0$ be as in Corollary 2.10. Then, for all $n \geq 0$, the dreadlock $f^{n}\left(G_{\underline{s}}\right)=G_{\sigma^{n}(\underline{s})}$ contains a point of modulus at most $R$. Hence the iterates of $f$ do not tend to infinity uniformly on $G_{\underline{s}}$.

The following is an alternative formulation of Definition 5.1, and allows us to connect the notion with our definition of criniferous functions.

Proposition 5.3 (Characterisation of hairs). A dreadlock $G_{\underline{s}}$ of $f$ is a hair if and only if, for every $z \in G_{\underline{s}}$, there is an arc connecting $z$ to $\infty$ on which $f^{n} \rightarrow \infty$ uniformly. 
Proof If $G_{\underline{s}}$ is a hair, then the stated condition holds by definition.

So now suppose that every point $z \in G_{\underline{s}}$ can be connected to infinity by an arc $\gamma_{z}$ on which the iterates tend to infinity uniformly. So $\gamma_{z} \subset \mu(z) \subset \mu_{\underline{s}} \subset G_{\underline{s}}$ by Lemma 4.8. By Lemma 4.9, the arcs $\gamma_{z}$ are linearly ordered by inclusion, and the $\operatorname{arc} \gamma_{z}$ is unique.

Let $\left(x_{n}\right)_{n=0}^{\infty}$ be a countable dense subset of $G_{\underline{s}}$. Set $n_{0}=0$ and define $n_{k+1}$ inductively as the minimal value of $n$ for which $x_{n}$ does not lie on the arc $\gamma_{x_{n_{k}}}$. (If no such $n$ exists, then $G_{\underline{s}}=\gamma_{x_{n_{k}}}$ satisfies Definition 5.1 (a) and we are done.) We set $y_{k}:=x_{n_{k}}$. Then the union of the arcs $\gamma_{y_{k}}$ is a single continuous injective curve $\gamma:(0, \infty) \rightarrow G_{\underline{s}}$, which can be parameterised such that $\gamma([1 / k, \infty))=\gamma_{y_{k}}$ for all $k$.

IF $\gamma(t)$ has a limit $z_{0} \in G_{\underline{s}}$ as $t \rightarrow 0$, then $\gamma_{z_{0}}=\gamma \cup\left\{z_{0}\right\}=\bar{\gamma}=G_{\underline{s}}$, since $\gamma$ is dense in $G_{\underline{s}}$. Hence $G_{\underline{s}}$ satisfies Definition 5.1 (a).

Otherwise, $\gamma((0, \infty)) \not \subset \gamma_{z}$ for all $z \in G_{\underline{s}}$. Hence we see that $\gamma_{z} \subset \gamma_{y_{k}}$ for sufficiently large $k$; it follows that $\gamma$ is surjective, and Definition 5.1 (b) holds.

Corollary 5.4 (Dreadlocks and criniferous functions). A transcendental entire function $f$ with bounded postsingular set is criniferous if and only if every dreadlock is a hair.

Proof Recall that every escaping point of $f$ belongs to some dreadlock. Hence the claim is immediate from Proposition 5.3.

Observe that we now have two apparently different definitions of periodic hairs, namely Definition 1.3, and the case of a periodic dreadlock (i.e., a dreadlock $G_{\underline{s}}$ at a periodic address $\underline{s}$ ) that is a hair. We conclude the section by showing that the two coincide.

Proposition 5.5 (Periodic hairs). Every periodic hair in the sense of Definition 1.3 is a periodic dreadlock, and this dreadlock is a hair in the sense of Definition 5.1. Conversely, any periodic dreadlock that is a hair has a parameterisation as a periodic hair in the sense of Definition 1.3.

Proof Suppose that $\gamma$ is a periodic hair. Then, for every $t \in \mathbb{R}$, the iterates $f^{n}$ tend to infinity uniformly on $\gamma([t, \infty))$. Hence, by Lemma 4.8, $\gamma$ is contained in a dreadlock $G_{\underline{s}}$, whose address $\underline{s}$ is necessarily periodic. Let $\mathcal{X}$ be as in Lemma 4.9. If $X \in \mathcal{X}$, then $f^{n}(X) \subset \gamma([0, \infty))$ for sufficiently large $n$, and hence $X \subset \gamma([-n, \infty))$. Thus we conclude that $\gamma=\mu_{\underline{s}}$.

Let $R$ be as in part ( $\overline{\mathrm{d}})$ of Theorem 2.5, applied to the set of fundamental domains occurring in $\underline{s}=F_{0} F_{1} F_{2} \ldots$ It follows that there is $t$ such that $\gamma((-\infty, t))$ contains only points of modulus less than $R$. Indeed, by choice of $R$ there is $n_{1}$ such that $f^{n}(z) \notin \gamma([0,1])$ for $n \geq n_{1}$, whenever $z \in G_{\underline{s}}$ with $|z| \geq R$; then we may take $t=1-n_{1}$.

Let $z \in G_{\underline{s}}$, and let $n_{0}$ be such that $f^{n}(z) \in F_{n}$ and $\left|f^{n}(z)\right|>R$ for $n \geq n_{0}$. We may assume that $n_{0}$ is a multiple of the period of $\underline{s}$. Recall that $f^{n_{0}}(z) \in G_{\underline{s}}$ is in the closure of $\gamma$ by Proposition 4.10. Since $\gamma((-\infty, t))$ contains no points of modulus 
greater than $R$, it follows that $f^{n_{0}}(z) \in \gamma([t, \infty))$. Thus $z \in \gamma\left(\left[t-n_{0}, \infty\right)\right)$. We have proved that $G_{\underline{s}}=\gamma$, and clearly $G_{\underline{s}}$ is a hair in the sense of Definition 5.1.

Now suppose that $\underline{s}$ is periodic and that $G_{\underline{s}}$ is a hair. Let $\tilde{\gamma}:(0, \infty) \rightarrow G_{\underline{s}}$ be a parameterisation of $G_{\underline{s}}$ as in part (b) of Definition 5.1. Consider the point $z_{0}:=\tilde{\gamma}(1)$, and its image $z_{1}=f\left(z_{0}\right)$, then $z_{1}=\tilde{\gamma}(t)$ for some $t$. It follows easily that $t>1$, and that the piece $\tilde{\gamma}((1, t))$ is disjoint from its forward and backward images in $G_{\underline{s}}$. We may reparameterise $\tilde{\gamma}$ to a curve $\gamma: \mathbb{R} \rightarrow G_{\underline{s}}$ such that $\gamma([0,1])$ corresponds to $\tilde{\gamma}([1, t])$, and such that $\gamma(t+1)=f(\gamma(t))$ for all $t$. Then $\gamma$ is a periodic hair in the sense of Definition 1.3.

REMARK 5.6 (On the concept of hairs). The notion of a "dynamic ray" of an entire function is given in [RRRS11, Definition 2.2]. By the same reasoning as in Observation 5.2, in our setting of postsingularly bounded functions this definition can be phrased as follows: a "dynamic ray" of a postsingularly bounded entire function is a maximal curve in the escaping set satisfying (b) of Definition 5.1. Hence every dynamic ray is contained in the core of a dreadlock, and a dreadlock $G_{s}$ is a dynamic ray if and only if it satisfies Definition 5.1 (b). In particular, for a bounded address $\underline{s}, G_{\underline{s}}$ is a dynamic ray if and only if it is a hair. Moreover, for criniferous functions - and in particular the class of functions for which hairs are constructed in [RRRS11] - every dynamic ray in the sense of [RRRS11, Definition 2.2] is either a hair in the sense of Definition 5.1, or becomes such upon the addition of a finite escaping endpoint.

In general, [RRRS11] leaves open the possibility that a dreadlock contains a hair as a proper subset. For example, it follows from [Rem16, Theorem 2.5] that there is an entire function $f$ with bounded postsingular set (and indeed of disjoint type), having a bounded-address dreadlock $G_{s}$ with the following properties. The set $\overline{G_{\underline{s}}} \backslash G_{\underline{s}}$ consists of a single point $z_{0}$ with bounded orbit, and $\hat{G}_{\underline{s}}$ is homeomorphic to a $\sin (1 / x)$ continuum, with the starting point of the accumulating curve corresponding to $\infty$, and one of the endpoints of the limiting interval situated at $z_{0}$. Then the accumulating curve itself is a "dynamic ray", but this ray does not include all points of $G_{\underline{s}}$.

Here, we restrict only to consider cases where the entire dreadlock $G_{\underline{s}}$ is a hair, and hence leave open the question of whether proper subsets of dreadlocks should be considered "hairs" or not.

Also note that [RRS10, p. 740] defines a notion of Devaney hairs; for postsingularly bounded functions such a Devaney hair is a curve $\gamma$ as in (b) of Definition 5.1, with the addition of a finite, not necesarily escaping, endpoint. In particular, if $G_{\underline{s}}$ is a hair, then $G_{\underline{s}}$ contains many Devaney hairs in the sense of [RRS10], linearly ordered by inclusion. Conversely, any Devaney hair is either contained in a dreadlock, or consists of a dreadlock together with a finite landing point. 


\section{Accumulation Sets and Landing Properties of Bounded-Address Dreadlocks}

We now study the accumulation behaviour of dreadlocks at bounded external addresses. (The restriction to bounded addresses is due to the phenomena discussed at the end of Remark 4.15.) In the case where the dreadlock in question is a hair, it is clear that the "accumulation set" of this dreadlock should be the set of limit points of the curve $\gamma(t)$ as $t \rightarrow 0$, where $\gamma$ is the curve from Definition 5.1. For periodic hairs, one can see easily that this is equivalent to fixing a base point on this hair, considering its successive preimages along $\gamma$, and studying the accumulation set of this sequence. This motivates the following definition.

DeFINITION 6.1 (Accumulation sets of bounded addresses). Let $\underline{s}$ be a bounded external address, and let $\zeta \in W_{0}$.

For $n \geq 1$, set $\zeta_{n}:=\zeta_{n}(\underline{s}):=f_{s}^{-n}(\zeta) \in \tau_{n}(\underline{s})$. (Recall from Definition 3.8 that $f_{\underline{s}}^{-n}=\left(\left.f^{n}\right|_{\tau_{n}(\underline{s})}\right)^{-1}$.) Then the accumulation set $\Lambda(\underline{s}, \zeta)$ of $\underline{s}$ with respect to $\zeta$ is defined to be the accumulation set (in $\hat{\mathbb{C}}$ ) of the sequence $\left(\zeta_{n}\right)_{n=1}^{\infty}$.

Note that this is an abstract definition of the accumulation set associated to an address; it uses only the notion of fundamental tails, and does not require the definition of dreadlocks or their properties. That this is a natural concept may not be clear a priori, but should become apparent through the results proved in this section. We begin by verifying that $\Lambda(\underline{s}, \zeta)$ is independent of $\zeta$.

This follows from the following well-known fact about the shrinking of univalent preimages. (Compare also [Lyu83, Proposition 3].) Recall that $\Omega$ is the unique unbounded component of $\mathbb{C} \backslash P(f)$.

Lemma 6.2 (Euclidean shrinking). Suppose that $V \Subset \Omega$ is a bounded Jordan domain. Then, for any $\varepsilon>0$ and any compact set $K \subset \mathbb{C}$, there exists $N_{\varepsilon}$ with the following property. For $n \geq N_{\varepsilon}$, every connected component of $f^{-n}(V)$ that intersects $K$ has Euclidean diameter at most $\varepsilon$.

Proof Suppose, by contradiction, that there is a sequence $\left(V_{k}\right)_{k=0}^{\infty}$ of $n_{k}$-th preimages of $V$, with $n_{k} \rightarrow \infty$, with $V_{k} \cap K \neq \emptyset$ and $\inf _{k} \operatorname{diam}\left(V_{k}\right)>0$. Let $\tilde{V} \Subset \Omega$ be a slightly larger Jordan domain than $V$ with $\bar{V} \subset \tilde{V}$, and let $\tilde{V}_{k}$ be the component of $f^{-n_{k}}(\tilde{V})$ containing $V_{n_{k}}$. Then $f^{n_{k}}: \tilde{V}_{k} \rightarrow \tilde{V}$ is a covering map and hence a conformal isomorphism, whose inverse $\varphi_{k}:=\left(\left.f\right|_{\tilde{V}_{k}}\right)^{-1}: \tilde{V} \rightarrow \tilde{V}_{k}$ maps $V$ to $V_{k}$.

By assumption, there is a sequence $\left(z_{k}\right)_{k=0}^{\infty}$ with $z_{k} \in K \cap V_{k}$. By Koebe's distortion theorem, $\tilde{V}_{k}$ contains a round disc around $z_{k}$ whose diameter is comparable to that of $V_{k}$. By assumption, the latter is bounded from below. Hence, if $U$ is a sufficiently small disc centred at a limit point of the sequence $\left(z_{k}\right)$, then $U$ is contained in infinitely many $\tilde{V}_{n_{k}}$. It follows that $f^{n}(U) \subset \Omega$ for all $n \geq 0$, and $f^{n}(U) \subset \tilde{V}$ for infinitely many $n$. This contradicts Corollary 3.3. 
Corollary 6.3 (Spherical shrinking). Suppose that $V \Subset \Omega$ is a bounded Jordan domain. Then, for any $\varepsilon>0$ there exists $N$ such that for $n \geq N$ every connected component of $f^{-n}(V)$ has spherical diameter at most $\varepsilon$.

Proof Let $K \subset \mathbb{C}$ be a compact set whose complement has spherical diameter less than $\varepsilon$. Since the spherical and Euclidean metrics are comparable on any compact subset of the plane, there is $\varepsilon_{1}$ such that any set of Euclidean diameter at most $\varepsilon_{1}$ that intersect $K$ has spherical diameter at most $\varepsilon$. Let $N=N_{\varepsilon_{1}}$ be as in Lemma 6.2, let $n \geq N$, and let $X$ be a connected component of $f^{-n}(V)$. Then either $X \cap K=\emptyset$, and hence $X$ has spherical diameter less than $\varepsilon$, or $X \cap K \neq \emptyset$ and Lemma 6.2 applies. In the latter case, $\operatorname{diam} X \leq \varepsilon_{1}$, and hence $X$ also has spherical diameter at most $\varepsilon$ by choice of $\varepsilon_{1}$.

COROllary AND Definition 6.4 (Accumulation sets and landing of dreadlocks). Let $\underline{s}$ be a bounded external address. Then $\Lambda\left(\underline{s}, \zeta^{1}\right)=\Lambda\left(\underline{s}, \zeta^{2}\right)$ for any two choices of $\zeta^{1}, \zeta^{2} \in W_{0}$.

We write $\Lambda\left(G_{\underline{s}}\right):=\Lambda(\underline{s}):=\Lambda(\underline{s}, \zeta)$, and call $\Lambda\left(G_{\underline{s}}\right)$ the accumulation set of the dreadlock $G_{\underline{s}}$. The dreadlock $G_{\underline{s}}$ is said to land at a point $z_{0} \in \hat{\mathbb{C}}$ if $\Lambda\left(G_{\underline{s}}\right)=\left\{z_{0}\right\}$.

Proof Let $V$ be a bounded Jordan domain in $W_{0}$ containing both $\zeta^{1}$ and $\zeta^{2}$. For $j \in\{1,2\}$ and $n \geq 1$, write $\zeta_{j}^{n}:=f_{\underline{s}}^{-n}\left(\zeta^{j}\right) \in \tau_{n}:=\tau_{n}(\underline{s})$. Since $f^{n}: \tau_{n} \rightarrow W_{0}$ is univalent, for any $n$ the points $\zeta_{n}^{1}$ and $\zeta_{n}^{2}$ belong to the same connected component of $f^{-n}(V)$. By Corollary 6.3, the spherical diameter of this component tends to zero as $n \rightarrow \infty$. Hence the sequences $\left(\zeta_{n}^{1}\right)_{n=1}^{\infty}$ and $\left(\zeta_{n}^{2}\right)_{n=1}^{\infty}$ have the same accumulation set in $\hat{\mathbb{C}}$.

The following establishes a number of fundamental properties of accumulation sets.

Proposition 6.5 (Properties of accumulation sets). Let $\underline{s}$ be a bounded external address, and $G_{\underline{s}}$ the dreadlock at address $\underline{s}$.

(a) The accumulation set $\Lambda(\underline{s})$ is a nonempty connected subset of $\hat{\mathbb{C}}$.

(b) The closure $\hat{G}_{\underline{s}}$ of $G_{\underline{s}}$ in $\hat{\mathbb{C}}$ is given precisely by

$$
\hat{G}_{\underline{s}}=G_{\underline{s}} \cup \Lambda(\underline{s}) \cup\{\infty\} .
$$

(c) If $J_{\underline{s}} \backslash G_{\underline{s}} \neq \emptyset$, then this set has a unique element $z_{0}$, and $G_{\underline{s}}$ lands at $z_{0}$, which has bounded orbit.

(d) If $\underline{s}$ is of disjoint type, then $G_{\underline{s}}$ lands at a point $z_{0} \in J_{\underline{s}}$ having bounded orbit.

(e) Let $U$ be a neighbourhood of a point $z_{0} \in \Lambda(\underline{s})$. Then $f^{n}$ does not tend to infinity uniformly on $U \cap G_{\underline{s}}$.

(f) Let $K \subset \hat{G}_{\underline{s}}$ be compact with $K \cap \Lambda(\underline{s})=\emptyset$. Then $f^{n} \rightarrow \infty$ uniformly on $K \cap \mathbb{C}$, and in particular $K \cap \mathbb{C} \subset J_{\underline{s}}$. 
Before proving these facts, we observe a few consequences. Firstly, we see that we can characterise the accumulation set purely in terms of $G_{\underline{s}}$ as a subset of the escaping set, justifying the notation $\Lambda\left(G_{\underline{s}}\right)=\Lambda(\underline{s})$.

COROLlary 6.6 (Topological landing criterion). Let $G_{s}$ be a dreadlock at a bounded address $\underline{s}$. Then the accumulation set of $G_{\underline{s}}$ consists precisely of those points in $\hat{G}_{\underline{s}}$ having a neighbourhood $U$ such that $f^{n}$ does not tend to infinity uniformly on $U \cap G_{\underline{s}}$.

In particular, $G_{\underline{s}}$ lands at a point $z_{0} \in \mathbb{C}$ if and only if $\hat{G}_{\underline{s}}=G_{\underline{s}} \cup\left\{z_{0}, \infty\right\}$ and if furthermore, for every neighbourhood $U$ of $z_{0}$ in $\hat{\mathbb{C}}$, the iterates of $f$ tend to infinity uniformly on $G_{\underline{s}} \backslash U$.

Proof of Corollary 6.6, using Proposition 6.5 The first claim is a direct consequence of Proposition 6.5 (e) and (f). The second claim follows from the first (together with the fact that the accumulation set of $G_{\underline{s}}$ is nonempty).

In particular, it follows that for hairs our definition of the accumulation set agrees with the usual one. Recall by Observation 5.2 that if a bounded-address dreadlock is a hair, it must satisfy part (b) of Definition 5.1.

Corollary 6.7 (Accumulation sets of hairs). Suppose that a dreadlock $G_{\underline{s}}$ at bounded address is a hair, and let $\gamma:(0, \infty) \rightarrow G_{\underline{s}}$ be the continuous bijection from Definition 5.1. Then $\Lambda\left(G_{\underline{s}}\right)$ is precisely the accumulation set of $\gamma(t)$ as $t \rightarrow 0$.

Proof of Corollary 6.7, using Proposition 6.5 Let $\Lambda(\gamma) \subset \hat{\mathbb{C}}$ denote the set of accumulation points of $\gamma(t)$ as $t \rightarrow 0$. Recall that the iterates of $f$ tend to infinity uniformly on $\gamma([t, \infty))$ for all $t>0$, by Definition 5.1 . Thus we see from part (e) of Proposition 6.5 (or from Corollary 6.6) that $\Lambda\left(G_{\underline{s}}\right) \subset \Lambda(\gamma)$.

It remains to prove the opposite inclusion, $\Lambda(\gamma) \subset \Lambda\left(G_{\underline{s}}\right)$. If $\gamma$ lands, i.e., when $\# \Lambda(\gamma)=1$, this follows from the first inclusion and the fact that $\Lambda\left(G_{\underline{s}}\right) \neq \emptyset$. So we may assume that $\Lambda(\gamma)$ is a nondegenerate continuum.

Since $\Lambda(\gamma) \subset \hat{G}_{\underline{s}}$, we have

$$
\tilde{\Lambda}:=\Lambda(\gamma) \backslash\left(G_{\underline{s}} \cup\{\infty\}\right) \subset \Lambda\left(G_{\underline{s}}\right)
$$

by (b) of Proposition 6.5. As $\Lambda\left(G_{\underline{s}}\right)$ is closed, it thus suffices to prove that $\tilde{\Lambda}$ is dense in $\Lambda(\gamma)$.

This is clear if $\Lambda(\gamma) \cap G_{\underline{s}}=\emptyset$. Otherwise, let $t_{0}>0$ be such that $\gamma\left(t_{0}\right) \in \Lambda(\gamma)$. We claim that $\gamma\left(\left(0, t_{0}\right]\right) \subset \bar{\Lambda}(\gamma)$.

This follows from a well-known argument that we sketch as follows; compare [Rem06b, Lemma 5.1] for the case of exponential maps. For every $t>0$, there are pieces of other hairs of $f$ accumulating uniformly from above and below on $\gamma([t, \infty))$. (For example, this follows from [Rem16, Proposition 8.1] via [Rem09, Theorem 1.1].) Thus, in order to accumulate on $\gamma\left(t_{0}\right)$, the curve $\gamma$ must also accumulate on $\gamma\left(\left[t, t_{0}\right]\right)$; letting $t \rightarrow 0$, the claim is established. 
Let $t_{0} \in(0, \infty]$ be the supremum over all possible choices of $t_{0}$, and let $\left(t_{n}\right)_{n=0}^{\infty}$ be a decreasing sequence with $t_{n} \rightarrow 0$. Then $A_{n}:=\gamma\left(\left[t_{n}, t_{0}\right]\right)$ is a compact and nowhere dense subset of $\Lambda(\gamma)$ for all $n$. By Baire's theorem,

$$
\tilde{\Lambda}=\Lambda(\gamma) \backslash \bigcup A_{n}
$$

is dense in $\Lambda(\gamma)$, as claimed. This completes the proof.

REMARK (REMARK 1) The proof shows that a hair $G_{s}$ either lands, or otherwise a generic point in $\Lambda\left(G_{\underline{s}}\right)$ belongs to $\mathbb{C} \backslash G_{\underline{s}}$. It is plausible that this is true without the assumption that $G_{\underline{s}}$ is a hair, with a similar proof. This would simplify the characterisation in Corollary 6.6 as follows: A dreadlock $G_{s}$ at a bounded address $\underline{s}$ lands at a finite point $z_{0} \in \mathbb{C}$ if and only if $\overline{G_{\underline{s}}} \backslash G_{\underline{s}}=\left\{z_{0}\right\}$.

REMARK (REMARK 2) For periodic addresses, or for addresses satisfying a headstart condition as in [RRRS11], the proof of Corollary 6.7 is considerably simpler. Indeed, in this setting it is easy to see directly that the iterates of $f$ do not tend to infinity uniformly on any neighbourhood of any point of $\Lambda(\gamma)$.

Finally, we observe that most periodic rays land and most periodic points are landing points. Compare also [BK07, BF15, Ben16].

Corollary 6.8 (Most periodic dreadlocks land). Let $p \geq 1$. Then, for all but finitely many periodic addresses $\underline{s}$ of period $p$, the dreadlock $G_{\underline{s}}$ lands at a periodic point $z \in J_{s}$ of period $p$. Conversely, for all but finitely many periodic points $z$ of period $p$, the point $z$ is repelling and there is a periodic address $\underline{s}$ of period $p$ such that $z \in J_{\underline{s}}$; in particular, $G_{\underline{s}}$ lands at $z$.

Proof Passing to an iterate, we can assume that $p=1$. (Recall that the sets $J_{\underline{s}}$ are pairwise disjoint by Lemma 4.3 (a).) Only finitely many fundamental domains $F$ intersect $\bar{D}$, and hence all but finitely many fixed addresses of $f$ are of disjoint type. Hence the first claim follows from Proposition 6.5 (d). Similarly, all but finitely many fixed points of $f$ are contained in $W_{0}$, and hence have a fixed external address. So the second claim is a consequence of Proposition 6.5 (c).

The remainder of the section is dedicated to establishing Proposition 6.5. We shall do so by applying Corollary 6.3 to a suitable large Jordan domain $V$, depending on the collection of fundamental domains involved. The following technical lemma collects the properties that we require of $V$.

Lemma 6.9 (Domains for bounded-address dreadlocks). Let $\zeta$ belong to an unbounded connected component of $W_{0} \cap f^{-1}(D)$ and let $R>0$. Let $\mathcal{F}$ be any finite collection of fundamental domains of $f$. Then there is a Jordan domain $V \Subset \Omega$ with the following properties.

(i) $\zeta \in V$. 
(ii) For all $F \in \mathcal{F}$, the unique preimage $\zeta_{F}$ of $\zeta$ in $F$ also belongs to $V$.

(iii) For all $F \in \mathcal{F}$, there is a connected component $A_{F}$ of $V \cap \bar{F}$ containing $\zeta_{F}$ as well as all points of $\bar{F}$ having modulus at most $R$.

(iv) If $U$ is the connected component of $V \cap W_{0}$ containing $\zeta$, then $U \cap A_{F}$ intersects the unbounded connected component $\stackrel{\infty}{F}$ of $F \backslash \bar{D}$, for all $F \in \mathcal{F}$.

Proof See Figure 3. Write $\mathcal{F}=\left\{F^{1}, \ldots, F^{n}\right\}$. Let $\gamma$ be a Jordan curve in $\mathbb{C} \backslash \bar{D}$ that intersects the arc $\delta$ in exactly one point. We may assume that $\gamma$ is chosen so large that $\gamma$ surrounds $\zeta, f(\bar{D})$ and the set $\{f(z):|z| \leq R\}$.

For each $i$, set $\gamma^{i}:=f^{-1}(\gamma) \cap F^{i}$ and $\zeta^{i}:=\zeta_{F^{i}}$. Then $\gamma^{i}$ is a cross-cut of $F^{i}$ that separates $\zeta^{i}, F^{i} \cap \bar{D}$ and all points of modulus at most $R$ in $F^{i}$ from $\infty$ in $F^{i}$. In particular, $\gamma^{i}$ belongs to the unbounded connected component of $F^{i} \backslash \bar{D}$.

Let $X^{i}$ be the closure of the bounded component of $F^{i} \backslash \gamma^{i}$, and let $Y$ be the union of these components. Observe that different $X^{i}$ may intersect when the corresponding fundamental domains are adjacent. In this case, the corresponding two $X^{i}$ intersect precisely in a preimage component of the piece of $\delta$ that connects $\bar{D}$ to $\gamma$. Let $Y_{1}, \ldots, Y_{k}$ be the $k \leq n$ connected components of $X$, and set $\Gamma_{j}:=Y_{j} \cap f^{-1}(\gamma)$. That is, $\Gamma_{j}$ is the union of finitely many $\gamma^{i}$, together with their endpoints.

Then each $Y_{k}$ is a closed Jordan domain in $\Omega \backslash \delta$, and $\Gamma_{j}$ is an arc of $\partial Y_{k}$. (Recall that the closure of a fundamental domain does not meet $\delta$.) Let $\tilde{Y}$ be obtained from $Y$ by adding, for each $j \leq k$, an arc $\beta_{j}$ in $W_{0} \backslash X$ joining $\zeta$ to a point of $\Gamma_{j}$; this is possible by the assumption on $\zeta$. We may assume that two different $\beta_{j}$ intersect only at $\zeta$.

Then $\widetilde{Y}$ is a compact and full set, and we may let $V$ be a Jordan domain containing $\tilde{Y}$. The point $\zeta$ and all $\zeta^{i}$ belong to $V$ by construction. Moreover, each $Y_{j}$ is connected, and hence belongs to a single connected component of $V \cap\left(\bigcup_{i} \overline{F^{i}}\right)$. Finally, the union of all $\beta_{j}$ and all $\Gamma_{j}$ is connected by construction, is contained in $\mathbb{C} \backslash \bar{D}$ and intersects the unbounded connected component of $F^{i} \backslash \bar{D}$ for all $i$. This completes the construction.

The domain $V$ from Lemma 6.9 allows us to study the accumulation sets of dreadlocks. The following lemma is crucial not only in our study of accumulation sets, but also for the proofs of our main theorems. The key idea is that, in order to investigate the landing of a given dreadlock $G_{\underline{s}}$, we can study a certain chain of simply-connected domains (obtained as iterated preimages of the domain $V$ from Lemma 6.9) whose diameters shrink to zero.

LEMMA 6.10 (Preimage domains). Let $\mathcal{F}$ be a finite collection of fundamental domains of $f$, and assume that $\mathcal{F}$ contains every fundamental domain $F$ with $\bar{F} \cap \bar{D} \neq \emptyset$. Let $\zeta, R$ and $V$ be as in Lemma 6.9. If $R$ was chosen sufficiently large (depending only on $\mathcal{F})$, then the following holds.

Let $\underline{s}=F_{0} F_{1} \ldots$ be any external address. For $n \geq 1$, set $\zeta_{n}(\underline{s}):=f_{s}^{-n}(\zeta) \in \tau_{n}:=$ $\tau_{n}(\underline{s})$. Also let $V_{n}=V_{n}(\underline{s})$ be the unique component of $f^{-n}(V)$ containing $\zeta_{n}(\underline{s})$. Then the following hold for all $n \geq 1$. 


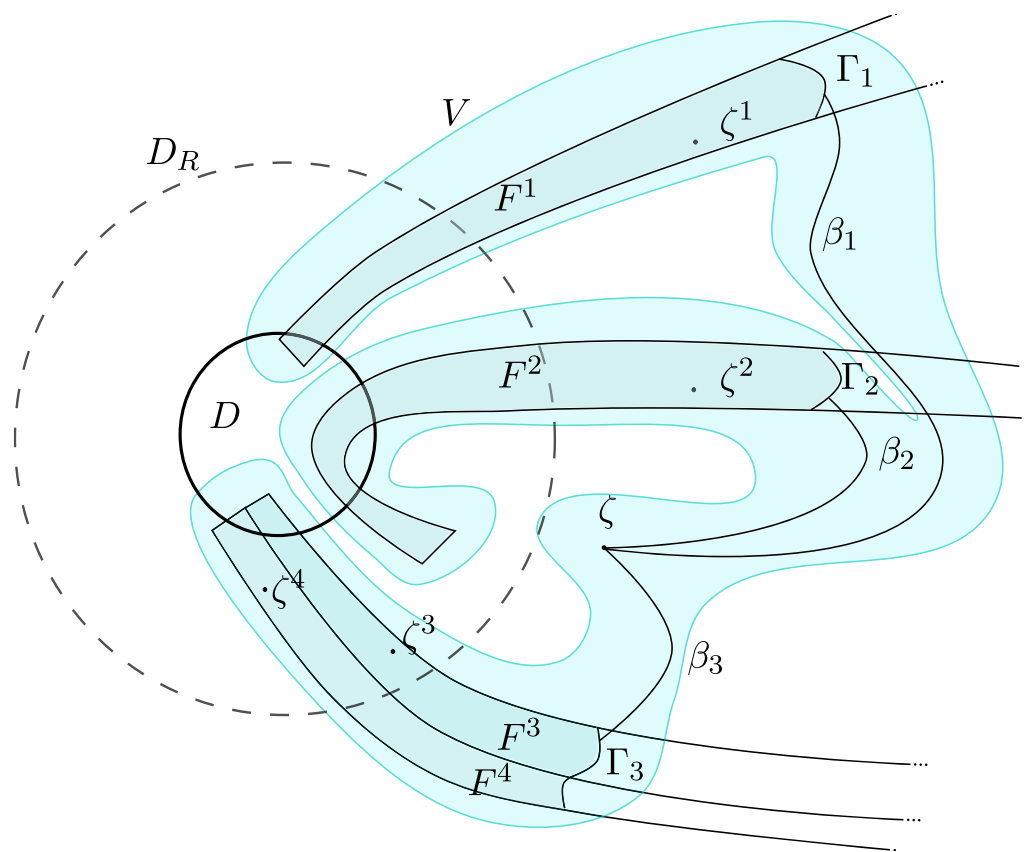

Figure 3: A domain $V$ as described in Lemma 6.9. The domain $V$ is shaded. For clarity, we include only the fundamental domains $F^{i}$ that comprise the collection $\tilde{F}$; each of these is contained in a tract of $f$ and is adjacent to other fundamental domains, which are not shown.

(1) The spherical diameter of $V_{n}(\underline{s})$ tends to 0 as $n \rightarrow \infty$. In particular, if $\left(\omega_{n}\right)_{n=0}^{\infty}$ is any sequence with $\omega_{n} \in V_{n}(\underline{s})$ for all sufficiently large $n$, then $\Lambda(\underline{s})$ coincides with the set $\Lambda_{\omega}$ of accumulation points of the sequence $\left(\omega_{n}\right)$.

(2) $\tau_{n+1}(\underline{s}) \subset \stackrel{\infty}{\tau}_{n+1}(\underline{s}) \cup V_{n}(\underline{s}) \subset \tau_{n}(\underline{s}) \cup V_{n}(\underline{s})$ and in fact $\overline{\tau_{n+1}(\underline{s})} \subset \tau_{n}(\underline{s}) \cup V_{n}(\underline{s})$.

(3) If $F_{n} \in \mathcal{F}$, then $\zeta_{n+1}(\underline{s}) \in V_{n}(\underline{s})$; in particular $V_{n}(\underline{s}) \cap V_{n+1}(\underline{s}) \neq \emptyset$.

If $F_{k} \in \mathcal{F}$ for all $k \geq 0$, then additionally:

(4) $V_{n}(\underline{s}) \cap \mu_{\underline{s}} \neq \emptyset$.

(5) $f^{m} \rightarrow \infty$ uniformly on $A_{n}:=G_{\underline{s}} \backslash \bigcup_{j \geq n} V_{j}(\underline{s})$. (In particular, $A_{n}$ is closed and $A_{n}=J_{\underline{s}} \backslash \bigcup_{j \geq n} V_{j}(\underline{s})$.) In fact, if $z$ belongs to this set, then $\left|f^{m}(z)\right| \geq \rho_{m-n}$ for $m \geq n$, where the sequence $\left(\rho_{\ell}\right)_{\ell=0}^{\infty}$ depends only on $\mathcal{F}$.

Proof By Corollary 6.3 the spherical diameter of $V_{n}(\underline{s})$ tends to 0 as $n \rightarrow \infty$, uniformly in $\underline{s}$. In particular, the set $\Lambda_{\omega}$ in (1) is independent of the choice of the sequence $\left(\omega_{n}\right)$. Also recall that $\zeta \in V$ by assumption; if we choose $\omega_{n}=\zeta_{n}$, then $\Lambda(\underline{s})=\Lambda(\underline{s}, \zeta)=\Lambda_{\omega}$. This proves (1).

Part (2) is trivial if $F_{n} \notin \mathcal{F}$. Indeed, by assumption on $\mathcal{F}$, we then have $\overline{F_{n}} \subset W_{0}$, $\tau_{n+1}(\underline{s})=\stackrel{\infty}{\tau}_{n+1}(\underline{s})$ and $\overline{\tau_{n+1}}(\underline{s}) \subset \tau_{n}(\underline{s})$ by definition. So suppose that $F_{n} \in \mathcal{F}$. Then $F_{n} \subset \stackrel{\infty}{F}_{n} \cup A_{F_{n}}$ and $\overline{F_{n}} \subset W_{0} \cup A_{F_{n}}$, where $A_{F_{n}} \subset V$ is the connected set from Lemma 6.9 (iii). 
Observe that $\tau_{n+1}(\underline{s})$ is the connected component of $f^{-n}\left(F_{n}\right)$ containing $\zeta_{n+1}(\underline{s})$, that $\tau_{n}(\underline{s})$ is the connected component of $f^{-n}\left(W_{0}\right)$ containing $\zeta_{n}(\underline{s})$, and that $V_{n}(\underline{s})$ is the connected component of $f^{-n}(V)$ containing $\zeta_{n}(\underline{s})$. Let $A_{1}$ be the connected component of $f^{-n}\left(A_{F_{n}}\right)$ contained in $V_{n}(\underline{s})$ and let $A_{2}$ be the connected component of $f^{-n}\left(A_{F_{n}}\right)$ contained in $\overline{\tau_{n+1}(\underline{s})}$. Since we have $\tau_{n+1}(\underline{s}) \subset \stackrel{\infty}{\tau}_{n+1}(\underline{s}) \cup A_{2}$ and $\overline{\tau_{n+1}(\underline{s})} \subset \tau_{n}(\underline{s}) \cup A_{2}$, we should show that $A_{1}=A_{2}$.

Let $x \in A_{F} \cap \stackrel{\infty}{F}_{n}$ be a point that can be connected to $\zeta$ in $W_{0} \cap V$. Such a point exists by Lemma 6.9 (iv). Let $x_{n}$ be the unique point of $f^{-n}(x)$ in $A_{1}$. Then $\zeta_{n}(\underline{s})$ and $x_{n}$ belong to the same connected component of $f^{-n}\left(W_{0}\right)$; i.e., $x_{n} \in \tau_{n}(\underline{s})$. Now $x \in \stackrel{\infty}{F}_{n}$, and $\stackrel{\infty}{\tau}_{n+1}(\underline{s})$ is the connected component of $f^{-n}\left(\stackrel{\infty}{F}_{n}\right)$ contained in $\tau_{n}(\underline{s})$. So $x_{n} \in \stackrel{\infty}{\tau}_{n+1}(\underline{s}) \subset \tau_{n+1}(\underline{s})$, and hence $x_{n} \in A_{2}$. We have shown $A_{1} \cap A_{2} \neq \emptyset$, and therefore $A_{1}=A_{2}$. Also $\zeta_{n+1}(\underline{s}) \in A_{2} \subset V$. We have proved both (2) and (3).

Now assume that all fundamental domains $F_{n}$ occurring in $\underline{s}=F_{0} \ldots F_{n-1} F_{n} \ldots$ belong to $\mathcal{F}$. Let $n \geq 0$.

We next prove (4). For $n \geq 0$, let $X_{n} \subset \mu_{\sigma^{n}(\underline{s})} \subset G_{\sigma^{n}(\underline{s})}$ be a closed unbounded connected set as in Theorem 2.5 (a). By Theorem 2.5 (c), if $R$ is large enough (depending only on $\mathcal{F}$ ), then $X_{n}$ can be chosen to contain a point of radius at most $R$. In particular, $X_{n}$ intersects the set $A_{F_{n}}$ from Lemma 6.9. So if $\tilde{X}_{n} \subset \mu_{\underline{s}}$ is the connected component of $f^{-n}\left(X_{n}\right)$ contained in $\tau_{n+1}(\underline{s})$, then $\tilde{X}_{n} \cap V_{n}(\underline{s}) \neq \underline{\emptyset}$.

To prove (5), suppose that $z \in A_{n}:=G_{\underline{s}} \backslash \bigcup_{j \geq n} V_{j}(\underline{s})$. Since $z \in G_{\underline{s}}$, there is $m_{0}$ such that $z \in \stackrel{\infty}{\tau}_{m}(\underline{s})$ for $m>m_{0}$. If $m_{0}$ is minimal with this property, then by (2),

$$
z \in \stackrel{\infty}{\tau}_{m_{0}+1}(\underline{s}) \backslash \stackrel{\infty}{\tau}_{m_{0}}(\underline{s}) \subset V_{m_{0}}(\underline{s}) .
$$

By assumption on $z$, we must have $m_{0}<n$. So, for $m \geq n$, we have $f^{m}(z) \in \stackrel{\infty}{F_{m}}$. Furthermore, by the proof of $(2), f^{m}(z) \notin A_{F_{m}}$, and thus $\left|f^{m}(z)\right|>R$.

So, if $R$ is chosen sufficiently large, $f^{n}\left(A_{n}\right)$ belongs to the set from Theorem $2.5(\mathrm{~d})$, on which the iterates tend to infinity uniformly and which depends only on $\mathcal{F}$. The same holds for $f^{n}\left(\overline{A_{n}}\right)$; in particular, this set is contained in $G_{\sigma^{m}(\underline{s})}$.

Proof of Proposition 6.5 Let $\mathcal{F}$ be a finite collection of fundamental domains containing all fundamental domains occurring in $\underline{s}$, and also all fundamental domains whose closure intersects $\bar{D}$. Let $\zeta, V$ and $V_{n}$ be as in Lemma 6.10. Here we assume that $R$ is chosen at least as large as the numbers from Theorem 2.5 (c) and (d).

Throughout the proof we will frequently refer to properties (1)-(5) of dreadlocks $G_{\underline{s}}$ with $\underline{s} \in \mathcal{F}^{\infty}$, as established in Lemma 6.10.

By (1),

$$
\Lambda(\underline{s})=\bigcap_{N \geq 1} \operatorname{cl}_{\widehat{\mathbb{C}}}\left\{\zeta_{n}(\underline{s}): n \geq N\right\}=\bigcap_{N \geq 1} \operatorname{cl}_{\widehat{\mathbb{C}}}\left(\bigcup_{n \geq N} V_{n}\right) .
$$

Each of the sets in the intersection on the right is compact and connected by (3); claim (a) follows. 
We next prove (e). First let $U$ be a neighbourhood of some point $z_{0} \in \Lambda(\underline{s})$. By (1), there are infinitely many $n$ such that $V_{n} \subset U$. By definition, all points in $V_{n}$ map to the bounded set $V$ after $n$ iterations, and $V_{n}$ contains a point of $\mu_{\underline{s}}$ by (4). Hence $f^{n}$ does not tend to infinity uniformly on $U \cap \mu_{\underline{s}} \subset U \cap G_{\underline{s}}$, as claimed. Observe that this argument also shows that

$$
\Lambda(\underline{s}) \subset \hat{G}_{\underline{s}} .
$$

On the other hand, let $K \subset \hat{G}_{\underline{s}}$ be compact with $K \cap \Lambda(\underline{s})=\emptyset$. Then there is $N \geq 1$ such that

$$
K \cap \overline{\bigcup_{n \geq N} V_{n}}=\emptyset .
$$

So $f^{n}$ tends to infinity uniformly on $K$ by (5), and in particular $K \subset J_{\underline{s}}$. This proves (f).

Now we establish the remaining claims in Proposition 6.5. Let $z \in \overline{G_{\underline{s}}} \backslash \Lambda(\underline{s})$. By (f), applied to $K=\{z\}$, we see that $z \in G_{\underline{s}}$. Thus

$$
\hat{G}_{\underline{s}}=\overline{G_{\underline{s}}} \cup\{\infty\} \subset G_{\underline{s}} \cup \Lambda(\underline{s}) \cup\{\infty\} .
$$

Together with (6.1), this proves (b).

Next suppose that $z_{0} \in J_{s} \backslash G_{s}$; that is, $z_{0}$ has address $\underline{s}$ but is not escaping. There is $n_{0}$ such that $f^{n}\left(z_{0}\right) \in J_{\sigma^{n}(z)}^{0}$ for $n \geq n_{0}$. Hence $\left|f^{n}\left(z_{0}\right)\right|<R$ by Theorem 2.5 (d). So $f^{n}\left(z_{0}\right) \in V$ for $n \geq n_{0}$, and thus $z_{0} \in V_{n}$. By (1), this proves $\Lambda(\underline{s})=\left\{z_{0}\right\}$, as claimed.

It remains to prove (d). Recall from Lemma 4.14 that, if $\underline{s}$ is of disjoint type, then $J_{\sigma^{n}(\underline{s})}=\overline{G_{\sigma^{n}(\underline{s})}} \subset F_{n}$ for all $n \geq 0$. Let $X$ be the set from 2.5 (d). Then there is $n_{0}$ such that $\left|f^{n}(z)\right|>R$ for all $n \geq n_{0}$ and all $z \in X$. Set

$$
\tilde{R}:=\max \left\{\left|f^{j}(z)\right|:|z| \leq R \text { and } j \leq n_{0}\right\} .
$$

By Theorem 2.5 (c), for all $n \geq 0$ there is $\zeta_{n} \in J_{\underline{s}}$ such that $\left|f^{n}\left(\zeta_{n}\right)\right| \leq R$. We claim that $\left|f^{j}\left(\zeta_{n}\right)\right| \leq \tilde{R}$ for all $n \geq n_{0}$ and all $j \leq n$. Indeed, let $j$ be minimal such that $\left|f^{j}\left(\zeta_{n}\right)\right|>R$ (if no such $j$ exists, there is nothing to prove). Then $f^{j}\left(\zeta_{n}\right) \in X$, and hence we must have $j>n-n_{0}$, and the claim follows by the definition of $\tilde{R}$.

Let $z_{0} \in J_{s}$ be a limit point of the sequence $\left(\zeta_{n}\right)$; then all points on the orbit of $z_{0}$ have modulus at most $\tilde{R}$. The claim now follows from (c).

REMARK 6.11 (Coding trees). Fix $\mathcal{F}, \zeta$ and $V$ as in Lemmas 6.9 and 6.10. For each $F \in \mathcal{F}$, we can choose an arc $\gamma_{F}$ connecting $\zeta$ to the point $\zeta_{F}$, and we may assume that these arcs are disjoint except at $\zeta$. For each $\zeta_{F_{0}}$ and each arc $\gamma_{F_{1}}$, there is a component of $f^{-1}\left(\gamma_{F_{1}}\right)$ connecting $\zeta_{F_{0}}$ to some point $\zeta_{F_{0} F_{1}}$ of $f^{-2}(\zeta)$. By Lemma 6.10, this is precisely the point contained in the fundamental tail at address $F_{0} F_{1}$.

Continuing inductively, we obtain an infinite tree with root $\zeta$, whose vertices of depth $n>0$ are the elements of $f^{-(n)}(\zeta)$ contained in fundamental domains of 
level $n-1$ whose addresses contain only entries from $\mathcal{F}$, and whose edges are all components of $f^{-n}\left(\gamma_{F}\right)$ for some $F \in \mathcal{F}$. Recall that the spherical length of these edges tends to zero as $n \rightarrow \infty$.

This tree can be considered to be an analogue of the geometric coding tree used by Przytycki [Prz94] in the case of rational functions. We see that, for each address $\underline{s}$ whose entries are drawn from $\mathcal{F}$, the accumulation set of the dreadlock $G_{\underline{s}}$ coincides precisely with the accumulation set of a branch of this coding tree. However, we will not use this language in the following.

\section{Separation Properties of Dreadlocks}

We now prove that a dreadlock that lands at a non-escaping point $z_{0} \in \mathbb{C}$ does not separate the plane. This fact is not used in our paper (except to deduce the corresponding parts of Theorems 1.1 and 1.5), but is important for applications.

Theorem 7.1 (Dreadlocks do not separate). Let $f$ be a postsingularly bounded function $f$, and let $\underline{s}$ be a bounded external address of $f$. Assume that $G_{\underline{s}}$ lands at a point $z_{0} \in \mathbb{C} \backslash G_{\underline{s}}$. Then $\overline{G_{\underline{s}}}$ does not separate the plane.

It is plausible that this can be directly deduced from our results and techniques in Section 6; indeed Pfrang does this for postsingularly finite $f$ [Pfr19]. Instead, we deduce Theorem 7.1 by relating landing dreadlocks to Julia continua of disjoint-type entire functions. Recall from Remark 4.15 that a function $g$ is of disjoint type if $g$ is hyperbolic with connected Fatou set, and that a Julia continuum of $g$ is a set of the form $\hat{C}=C \cup\{\infty\}$, where $C$ is a connected component of $J(g)$.

Theorem 7.2 (Dreadlocks and Julia continua). Let $f$ be a postsingularly bounded function, and let $\underline{s}$ be a bounded (resp. periodic) external address of $f$ such that $G_{\underline{s}}$ lands. If $\lambda$ is sufficiently small to ensure that $g: z \mapsto \lambda f(z)$ is of disjoint type, then $\hat{G}_{\underline{s}}$ is homeomorphic to a Julia continuum $\hat{C}$ of $g$ at a bounded (resp. periodic) external address.

The homeomorphism can be chosen to fix $\infty$, and send $z_{0}$ to the unique point of bounded orbit in $\hat{C}$.

Much is known about the topology of Julia continua of disjoint-type entire functions; see [Rem16]. Theorem 7.2 allows us to transfer this information to landing dreadlocks. In particular, we can easily deduce Theorem 7.1.

Proof of Theorem 7.1, using Theorem 7.2 Let $g$ be a disjoint-type function as in Theorem 7.2. Then the Fatou set $F(f)$ is connected and non-empty by definition. Hence $J(f)$ is a nowhere dense set that does not separate the plane, so no subset of $J(f)$ separates the plane.

So by Theorem 7.2 , the set $\hat{G}_{\underline{s}}$ is homeomorphic to a non-separating plane continuum. It is well-known that being a one-dimensional non-separating plane contin- 
uum is a topological property. (Indeed, a one-dimensional plane continuum is nonseparating if and only if it is tree-like; see [Bin51, Theorem 6] and [Mań12, Theorem 1.5]. Compare also [JT02].) So $\hat{G}_{\underline{s}}$ is also non-separating.

Proof of Theorem 7.2 By [BK07, Example on p. 392], for $\lambda \in \mathbb{C}$ small enough the function $g$ is indeed of disjoint type; we fix such $\lambda$ in the following. By [Rem09, Theorem 1.1], there is a map $\vartheta$ defined on

$$
J_{\geq R}(f):=\left\{z \in \mathbb{C}:\left|f^{n}(z)\right|>R \text { for all } n>1\right\},
$$

which is a conjugacy between $f$ on $J_{\geq R}(f)$ and $g$ on $\vartheta\left(J_{\geq R}(f)\right)$. Furthermore, $\vartheta$ extends continuously to $\infty$ with $\vartheta(\infty)=\infty$ - in particular, $\vartheta$ maps escaping points of $f$ to escaping points of $g-$ and $J_{\geq Q}(g) \subset \vartheta\left(J_{\geq R}(f)\right)$ for some $Q$ [Rem09, Lemma 3.3].

Assuming that $R>0$ is sufficiently large, the proof of [Rem09, Theorem 3.2] furthermore implies the following. For any external address address $\underline{s}$ of $f$, there is an external address $\underline{\tilde{s}}$ of $g$ such that

$$
\vartheta\left(J_{\underline{s}}^{0}(f) \cap J_{\geq R}(f)\right) \subset J_{\underline{\underline{s}}}^{0}(g) .
$$

If $\underline{s}$ is bounded (resp. periodic), then so is $\underline{\tilde{s}}$. We can extend $\left.\vartheta\right|_{J_{\geq R}(f) \cap I(f)}$ to a bijection $\vartheta: I(f) \cup\{\infty\} \rightarrow I(g) \cup\{\infty\}$ by defining

$$
\vartheta(z):=g_{\underline{s}}^{-n}\left(\vartheta\left(f^{n}(x)\right)\right) .
$$

The value $\vartheta(z)$ is independent of $n$ and, in particular, agrees with the original value when $z \in J_{\geq R}(f) \cup I(f)$. This follows from (7.1) and Proposition 4.4 and the conjugacy relation for $\vartheta$. Note that we do not claim that this bijection is continuous on $I(f)$.

Now suppose that $\underline{s}$ is bounded and that the dreadlock $G_{\underline{s}}(f)$ lands at a point $x_{0} \in \mathbb{C} \backslash G_{s}$. Note that the dreadlock $G_{\tilde{s}}$ of $g$ also lands at some point $y_{0} \in \mathbb{C}$ of bounded orbit since the function $g$ is of disjoint type. (Recall Proposition 6.5 (d).)

Consider the closures $\hat{G}_{\underline{s}}(f), \hat{G}_{\tilde{\underline{s}}}(g)$ of $G_{\underline{\underline{s}}}(f)$ and $G_{\tilde{\underline{s}}}(g)$ in $\hat{\mathbb{C}}$. For $n>=0$ define

$$
X_{n}:=f_{\underline{s}}^{-n}\left(J_{\sigma^{n}(\underline{s})}^{0}(f) \cap J_{\geq R}(f)\right) \cup\{\infty\} \subset \hat{G}_{\underline{s}} .
$$

By definition, $X_{n} \supset X_{n-1}$, and $\vartheta$ is continuous when restricted to $X_{n}$. We claim that $\vartheta$ is continuous on $X:=\bigcup_{n} X_{n}=G_{\underline{s}}(f) \cup\{\infty\}$.

Let $x \in X$. By assumption, $x \notin \Lambda(\underline{s})=\left\{x_{0}\right\}$. Hence by Corollary $6.6, z$ has a neighborhood $U$ in $X$ on which the iterates escape to infinity uniformly. Then, for sufficiently large $n, f^{n}(U) \subset J_{\underline{s}}^{0}(f)$, and hence $U \subset X_{n}$. Since $\vartheta$ is continuous on $X_{n}$ and $U$ is a neighbourhood of $x, \vartheta$ is continuous at $x$.

Moreover, $\vartheta^{-1}$ is continuous on the sets

$$
Y_{n}:=g_{\underline{s}}^{-n}\left(J_{\sigma^{n}(\underline{\underline{s}})}^{0}(g) \cap J_{\geq Q}(g)\right) \cup\{\infty\} .
$$

Hence, by the same argument as for $f$, the map $\vartheta^{-1}$ is continuous on the set $Y=G_{\tilde{\underline{s}}}(g) \cup\{\infty\}$, and $\vartheta: X \rightarrow Y$ is a homeomorphism. In particular, it extends to a homeomorphism between their respective one-point compactifications $\hat{G}_{\underline{s}}(f)=X \cup\left\{x_{0}\right\}$ and $\hat{G}_{\tilde{\underline{s}}}(g)=Y \cup\left\{y_{0}\right\}$. 
REMARK It is plausible that the homeomorphism in Theorem 7.2 is ambient; i.e., it extends to a homeomorphism of $\mathbb{C}$ onto itself.

\section{Landing Theorems for Dreadlocks}

With the definition of dreadlocks in Definition 4.2 and their accumulation sets in Definition 6.4 we can now state the main result of our paper. Recall that a dreadlock $G_{\underline{s}}$ is periodic if the address $\underline{s}$ is periodic under the shift map. Equivalently, $G_{\underline{s}}$ is periodic under the action of $f$ as a subset of $\mathbb{C}$.

Theorem 8.1 (Douady-Hubbard landing theorem for dreadlocks). Let $f$ be a transcendental entire function whose post-singular set $\mathcal{P}(f)$ is bounded.

Then every periodic dreadlock of $f$ lands at a repelling or parabolic periodic point, and conversely every repelling or parabolic periodic point of $f$ is the landing point of at least one and at most finitely many periodic dreadlocks.

We also obtain a corresponding theorem about landing properties at more general hyperbolic sets.

Theorem 8.2 (Landing at hyperbolic sets). Let $f$ be a transcendental entire function with bounded postsingular set. Moreover, suppose that $K$ is a hyperbolic set for $f$. Then every point $z \in K$ is the landing point of a dreadlock at some bounded external address $\underline{s}$; if $z$ is periodic, then so is $\underline{s}$.

In fact, we establish the following more precise version of Theorem 8.2. Note that the space of external addresses is equipped with the product topology; this is the same as the order topology arising from the cyclic order on addresses.

Theorem 8.3 (Accessibility of points in hyperbolic sets). Let $K$ be a hyperbolic set of a postsingularly bounded entire function $f$. Then there exists a compact and forward-invariant set $\mathcal{S}_{K}$ of bounded addresses of $f$, with the following properties.

(a) For every $\underline{s} \in \mathcal{S}_{K}$, the dreadlock at address $\underline{s}$ lands at a point $z_{0}(\underline{s}) \in K$.

(b) The function $\mathcal{S}_{K} \rightarrow K ; \underline{s} \mapsto z_{0}(\underline{s})$ is surjective and continuous. In particular, every point of $K$ is the landing point of a dreadlock.

(c) If $z_{0}$ is periodic, then all bounded-address dreadlocks landing at $z_{0}$ are periodic with the same period, and the number of such dreadlocks is finite.

(d) The dreadlocks at addresses in $\mathcal{S}_{K}$ land uniformly at $K$, in the following sense. Let $\zeta \in W_{0}$ and let $\varepsilon>0$. Then there is $n_{0}$ such that $\operatorname{dist}\left(\zeta_{n}(\underline{s}), K\right) \leq \varepsilon$ for all $n \geq n_{0}$ and all $\underline{s} \in \mathcal{S}_{K}$. (Recall from Definition 6.1 that $\zeta_{n}(\underline{s})=f_{\underline{s}}^{-n}(\zeta)$.)

Note that we do not claim that $\mathcal{S}_{K}$ can be chosen to consist of all addresses of dreadlocks landing at points of $K$. In particular, we do not prove that the function $\underline{s} \mapsto z_{0}(\underline{s})$ is continuous on the latter set.

The remainder of the paper will be dedicated to the proofs of these theorems. Let us first show that they imply the theorems stated in the introduction. 
Proof of Theorem 1.1, using Theorem 8.1 Let $\zeta$ be a repelling or parabolic periodic point. By Theorem 8.1, $\zeta$ is the landing point of a periodic dreadlock $G_{s}$. Let us set $A:=G_{\underline{s}}$, and let $p$ be the period of $\underline{s}$. Recall that $G_{\underline{s}} \subset I(f)$ by definition, and is unbounded and connected by Proposition 4.10. Since $G_{\underline{s}}$ lands at $\zeta$, we see from Corollary 6.6 that $\bar{A}=A \cup\{\zeta\}$ and that, for any neighborhood $U$ of $\zeta, f^{n} \rightarrow \infty$ uniformly on $A \backslash U$. Furthermore, by Theorem 7.1, $\bar{A}$ does not separate the plane. This proves that $A$ satisfies properties (a) and (c) of Theorem 1.1. Since $p$ is the period of $\underline{s}$, we have $f^{p}(A)=G_{\sigma^{p}(\underline{s})}=A$ and $f^{j}(A) \cap A=G_{\sigma^{j}(\underline{s})} \cap G_{\underline{s}}=\emptyset$ if $0<j<p$.

Proof of Theorem 1.4, using Theorem 8.1 Recall that every periodic hair is a periodic dreadlock (Proposition 5.5), and for criniferous functions, every periodic dreadlock is a periodic hair (Corollary 5.4). Moreover, by Corollary 6.7 such a dreadlock lands if and only if the corresponding hair lands in the sense of Definition 1.3.

Thus Theorem 1.4 follows immediately from Theorem 8.1.

Proof of Theorem 1.5, using Theorem 8.3 Define $\mathcal{A}:=\left\{G_{\underline{s}}: \underline{s} \in \mathcal{S}_{K}\right\}$, where $\mathcal{S}_{K}$ is as in Theorem 8.3. Properties (a), (c) and (e) in Theorem 1.5 follow immediately, as in the proof of Theorem 1.1. Furthermore, if $f$ is criniferous, then every element of $\mathcal{A}$ is an arc connecting its landing point $\zeta(A)$ to $\infty$, by Corollary 6.7.

Thus it remains to establish (d). Observe that, since $\mathcal{S}_{K}$ is compact and forwardinvariant, only finitely many different fundamental domains can occur within the addresses in $\mathcal{S}_{K}$. Let $\mathcal{F}$ be the collection of all these fundamental domains, as well as of all fundamental domains whose closure intersects $\bar{D}$. Let $V$ be as in Lemma 6.10, and let $n_{0}$ be as in part (d) of Theorem 8.3 for $\varepsilon / 2$. By Lemma 6.10 , we can find $n_{1} \geq n_{0}$ such that diam $V_{n}(\underline{s})<\varepsilon / 2$ for $n \geq n_{1}$. Thus $\operatorname{dist}(z, K)<\varepsilon$ for all $z \in V_{n}(\underline{s})$.

Let $z \in G_{\underline{s}}$ for some $\underline{s} \in \mathcal{S}_{K}$, and suppose that $\operatorname{dist}(z, K) \geq \varepsilon$. Then, by the above $z \in G_{\underline{s}} \backslash \bigcup_{j \geq n_{1}} V_{j}(\underline{s})$. By (5) of Lemma 6.10, the set of points with this property escape to infinity at a rate that depends only on $\mathcal{F}$ and on $n_{0}$ (and hence only on $\varepsilon)$. This completes the proof.

REMARK 8.4 (Shortcut to the landing theorems). Observe that Definition 6.1, concerning accumulation sets, requires only the definition of the fundamental tails $\tau_{n}(\underline{s})$ associated to an address $\underline{s}$, rather than any properties of the dreadlock $G_{\underline{s}}$ itself. Moreover, a key point in our proofs of Theorems 8.1, 8.2 and 8.3 is that we can ignore the fine structure of dreadlocks and use only the sets $V_{n}$ from Lemma 6.10. We only require properties (1), (2) and (3) of this lemma, all of which are likewise independent of the construction and analysis of dreadlocks in Sections 2, 4 and 5.

Hence it would be possible to prove these theorems without requiring any results from those sections, and using only the elementary parts of Section 6. Furthermore, while we used properties of dreadlocks above to deduce the statements of Theorems 1.1 and 1.5, many of these can be established more easily a posteriori for the dreadlocks under consideration, using the additional information that these dreadlocks land. Nonetheless, the general material concerning dreadlocks is crucial to the 
interpretation of our results as a natural extension of the classical Douady-Hubbard landing theorem for functions without hairs.

\section{Periodic Dreadlocks Land}

We now prove the first half of Theorem 8.1, which we restate here as follows.

Theorem 9.1 [(Landing of periodic dreadlocks)]. Let $f: \mathbb{C} \rightarrow \mathbb{C}$ be a transcendental entire function with bounded postsingular set.

Then every periodic dreadlock of $f$ lands at a repelling or parabolic periodic point of $f$, where the period of the landing point divides that of the dreadlock.

Proof of Theorem 9.1 Let $G_{\underline{s}}$ be a periodic dreadlock. Recall from Observation 4.13 that any dreadlock of $f$ is also a dreadlock of any iterate of $f$ (and vice versa). Hence, it is no loss of generality to assume that $\underline{s}$ is fixed by $\sigma$; i.e., $\underline{s}=F F F F \ldots$, where $F$ is a fundamental domain of $f$.

Fix $\zeta$ and $V$ as in Lemmas 6.9 and 6.10. Let $\tau_{n}$ be the fundamental tail of level $n$ associated to $\underline{s}$, and let $\zeta_{n}$ be the unique element of $f^{-n}(\zeta)$ in $\tau_{n}$.

Recall that $\zeta_{1}=\zeta_{F} \in V$, and let $\gamma:[0,1] \rightarrow V$ be a rectifiable curve with $\gamma(0)=\zeta_{1}$ and $\gamma(1)=\zeta$. Then, by Lemma 6.10 , for every $n \geq 1$ there is a component $\gamma_{n}$ of $f^{-n}(\gamma)$ connecting $\zeta_{n}$ and $\zeta_{n+1}$. We can hence combine these to a continuous curve $\gamma:[-\infty, 1] \rightarrow \mathbb{C}$, with $f(\gamma(t))=\gamma(t+1)$ for $t \leq 0$, where $\left.\gamma\right|_{[0,1]}=\gamma$, and $\left.\gamma\right|_{[-n, 1-n]}=\gamma_{n}$.

By Lemma $6.10(1)$, the spherical diameter of $\gamma_{n}$ tends to zero as $n \rightarrow \infty$. Therefore the set $\Lambda(\gamma)$ of accumulation points of $\gamma(t)$ as $t \rightarrow-\infty$ is precisely the accumulation set $\Lambda\left(G_{\underline{s}}\right)=\Lambda(\underline{s}, \zeta)$ of $G_{\underline{s}}$ in the sense of Definition 6.1.

Claim As $t \rightarrow-\infty, \gamma(t)$ converges to a fixed point of $f$.

Proof Re call that the spherical distance between $\zeta_{n}$ and $\zeta_{n+1}$ tends to zero as $n \rightarrow \infty$, and $f\left(\zeta_{n+1}\right)=\zeta_{n}$. Hence, by continuity, any finite point of $\Lambda(\gamma)=\Lambda(\underline{s}, \zeta)$ is a fixed point of $f$. Since the set of fixed points is discrete in $\mathbb{C}$, and $\Lambda(\gamma)$ is connected, the latter set is a singleton, whose sole element is either a fixed point or $\infty$. We must exclude the second possibility.

So suppose, by contradiction, that $\gamma(t) \rightarrow \infty$ as $t \rightarrow-\infty$. Recall that $\Omega$ is the unbounded connected component of $\mathbb{C} \backslash \mathcal{P}(f)$. Let $\rho_{n}:=\ell_{\Omega}\left(\gamma_{n}\right)$ be the hyperbolic length of $\gamma_{n}$ in $\Omega$. Since $\gamma_{n} \rightarrow \infty$ and the postsingular set is bounded, formula (3.2) of Proposition 3.1 implies that

$$
\rho_{n+1} \leq \rho_{n} / 2
$$

for all sufficiently large $n$. It follows that the hyperbolic length of $\gamma$ is bounded. As the hyperbolic metric on $\Omega$ is complete, this contradicts our assumption that $\gamma$ tends to $\infty$. 
The classical snail lemma of Douady and Sullivan (see [DH85, Exposé VIII, Proposition 2, p. 60] or [Mil06, Lemma 16.2]) shows that the limit point of $\gamma$ is either repelling or parabolic with multiplier 1 , and the proof is complete.

REMARK In fact, the above claim follows already from [Rem08, Theorems B.1 and B.2]. Here Theorem B.1 is a hyperbolic expansion argument going back to the proof by Douady and Hubbard [DH85, Exposé VIII] of the first half of the landing theorem for polynomials. On the other hand, the proof of [Rem08, Theorem B.2], which shows that $\gamma(t)$ cannot converge to infinity as $t \rightarrow-\infty$, used the notion of "extendability", which was developed for more general purposes in [Rem08]. For the reader's convenience, we gave the complete and much simpler proof above, in the spirit of Deniz [Den14].

It is possible that the landing point in Theorem 9.1 is also the landing point of other dreadlocks. However, as we now observe, there can only be finitely many of these, and they all need to be periodic. The idea of the proof is very similar to the polynomial case [Mil06, Lemma 18.12], but we need to take into account the non-compactness of the space of addresses. Compare also [RS08a, Lemma 3.2] for the case of exponential maps.

LEMMA 9.2 Let $f$ be a transcendental entire function with bounded postsingular set, and let $z$ be the landing point of a dreadlock with periodic address. Then the number of bounded-address dreadlocks landing at $z$ is finite, and their addresses are all periodic of the same period.

Proof Let $\underline{s}^{0}$ be the address of the periodic dreadlock landing at $z$; we may assume that its period $p$ is minimal with this property. Replacing $f$ by $f^{p}$, we may then assume that $p=1$. Let $\mathcal{S}_{z}$ be the set of bounded external addresses $\underline{s}$ for which $G_{\underline{s}}$ lands at $z$.

Claim There is a finite collection $\mathcal{F}$ of fundamental domains with the following property. Every address $\underline{s} \in \mathcal{S}_{z}$ contains some element of $\mathcal{F}$ infinitely many times.

Proof Let $\mathcal{F}$ consist of all fundamental domains $F$ such that either $\bar{D} \cap \bar{F} \neq \emptyset$, or $z \in F$. Clearly $\mathcal{F}$ is finite. Suppose that $\underline{s}=F_{0} F_{1} F_{2} \ldots$ is such that $F_{n} \notin \bar{F}$ for $n \geq n_{0}$. Then $\sigma^{n_{0}}(\underline{s})$ is of disjoint type, and $z \notin F_{n_{0}} \supset f^{n_{0}}\left(\overline{G_{\underline{s}}}\right)$. In particular, $\underline{s}$ does not land at $z$.

So any address in $\mathcal{S}_{z}$ contains infinitely many entries from $\mathcal{F}$; since the latter set is finite, at least one of these is itself repeated infinitely many times.

Since $f$ maps a neighborhood of $z$ to another neighborhood of $z$ as an orientationpreserving homeomorphism, it preserves the cyclic order of the dreadlocks landing at $z$. As remarked at the end of Section 13, this implies that $f$ also preserves the cyclic order of these dreadlocks at $\infty$. In other words, the shift map $\sigma: \mathcal{S}_{z} \rightarrow \mathcal{S}_{z}$ is injective and preserves the cyclic order of addresses on $\mathcal{S}_{z}$. 
Recall that $\underline{s}^{0}$ is a fixed address, say $\underline{s}^{0}=F^{0} F^{0} F^{0} \ldots$ Hence $\sigma$ also preserves the (linear) order $<$, where $\underline{s}<\underline{\tilde{s}}$ means that $\underline{s}^{0} \prec \underline{s} \prec \underline{\tilde{s}}$ in the cyclic ordering. So if $\underline{s}=F_{0} F_{1} F_{2} \ldots$ is an element of $\mathcal{S}_{z}$, then $\left(\sigma^{n}(\underline{s})\right)_{n=0}^{\infty}$ is monotone. If $F \in \mathcal{F}$ is the domain from the claim above, then we clearly must have $F_{n}=F$ for all sufficiently large $n$. By injectivity of $\sigma$, we conclude that $\underline{s}=F F F \ldots$ is itself a fixed address. As $\mathcal{F}$ is finite, the proof is complete.

REMARK 9.3 Recall that we defined dreadlocks only for maps with bounded postsingular sets, and landing of dreadlocks only for dreadlocks at bounded addresses. This allows us to state the lemma in the above simple form, which is all that will be required for the purpose of our main results.

However, observe that the proof of the lemma is purely combinatorial, and does not utilise either assumption in an essential manner. In particular, let $f \in \mathcal{B}$ be arbitrary (not necessarily with bounded postsingular set), and let $z_{0} \in J(f)$ be a fixed point of $f$. Suppose that $\mathcal{S}_{z}$ is a forward-invariant set of external addresses $\underline{s}$ of $f$, and that for every $\underline{s} \in \mathcal{S}$ there is an unbounded connected set $A_{\underline{s}}$ with the following properties:

- the cyclic order of the sets $A_{\underline{s}}$ at infinity agrees with the cyclic order of their external addresses;

- $z_{0} \in \overline{A_{\underline{s}}}$ for all $\underline{s} \in \mathcal{S}_{z}$;

- $\overline{A_{\underline{s}}} \cap \underline{A_{\underline{s}}}=\emptyset$ for $\underline{s} \neq \underline{\tilde{s}}$;

- $\overline{A_{\underline{s}}}$ does not separate the plane;

- $f\left(A_{\underline{s}}\right)=A_{\sigma(\underline{s})}$.

If $\mathcal{S}$ contains a periodic element, then it follows as above that $\mathcal{S}_{z}$ is finite and contains only periodic addresses. In particular, for any $f \in \mathcal{B}$, the landing point of a periodic hair cannot be the landing point of a non-periodic hair.

\section{Landing at Hyperbolic Sets}

Proof of Theorem 8.3 Let $K$ be a hyperbolic set of $f$. Replacing $f$ by a sufficiently high iterate, there is a neighbourhood $U$ of $K$ such that $\left|f^{\prime}(z)\right| \geq 2$ for all $z \in U$. (It is easy to see that proving the theorem for an iterate of $f$ also establishes it for $f$ itself; we leave the details to the reader.) We may additionally assume that the disc $D$ in the definition of fundamental domains is chosen so large that $K \subset D$. Finally, by Corollary 6.3, it is enough to prove (d) for some specific choice of $\zeta \in W_{0}$. Therefore we may fix $\zeta$ belonging to an unbounded connected component of $W_{0} \cap f^{-1}(D)$, as required in the hypothesis of Lemma 6.9.

Set $\delta:=\operatorname{dist}(K, \partial U)$. For $z \in K$, define $B_{0}(z):=B(z, \delta)$, and let $B_{n}(z)$ denote the connected component of $f^{-n}\left(B_{0}\left(f^{n}(z)\right)\right)$ containing $z$. Then $\overline{B_{n+1}(z)} \subset B_{n}(z)$ for all $n$, and $f: B_{n+1}(z) \rightarrow B_{n}(f(z))$ is a conformal isomorphism. For $n \geq 0$, define

$$
U_{n}:=\bigcup_{z \in K} B_{n}(z) .
$$


Then $\overline{U_{n+1}} \subset U_{n} \subset U$ and $f\left(U_{n+1}\right)=U_{n}$ for all $n$. Clearly $K=\bigcap_{n \geq 0} \overline{U_{n}}=\bigcap_{n \geq 0} U_{n}$. By the blowing-up property of the Julia set (see e.g. [Bak84, Lemma 2.2]), and compactness of $K$, there is some $N_{1}$ with the following property: if $n \geq N_{1}$ and $z \in K$, then $f^{-n}(\zeta) \cap B_{0}(z) \neq \emptyset$. In particular, for all such $n$ and $z$ there is a finite external address of length $n$ such that $\zeta_{n}(\underline{s}) \in B_{0}(z)$, and hence $\tau_{n}(\underline{s}) \cap B_{0}(z) \neq \emptyset$. Define

$$
\varepsilon:=\frac{\delta}{2} \leq \min _{z \in K} \operatorname{dist}\left(\partial B_{0}(z), \overline{B_{1}(z)}\right) .
$$

Now let $V$ be as in Lemma 6.9 for the finite collection $\mathcal{F}$ of fundamental domains that intersect $D$. Similarly as in Lemma 6.10 , if $\underline{s}$ is an infinite external address or a finite external address of length at least $n$, we define $V_{n}(\underline{s})$ to be the unique component of $f^{-n}(V)$ containing $\zeta_{n}(\underline{s}):=f_{s}^{-n}(\zeta) \in \tau_{n}(\underline{s})$.

By Lemma 6.2, there exists $N \geq N_{1}$ with the following property. Suppose that $n \geq N$ and that $\underline{s}$ is a finite or infinite external address of length at least $n$ with $V_{n}(\underline{s}) \cap \overline{U_{0}} \neq \emptyset$. Then

$$
\operatorname{diam} V_{n}(\underline{s})<\varepsilon .
$$

For $n \geq N$ and $z \in K$, we now define $\mathcal{S}_{n}(z)$ to be the set of finite external addresses $\underline{s}$ of length $n$ for which the tail $\tau_{n}(\underline{s})$ intersects $B_{n-N}(z)$. Observe that

$$
\mathcal{S}_{n}:=\bigcup_{z \in K} \mathcal{S}_{n}(z)
$$

is finite for every $n$ by Lemma 2.1. We also define $\mathcal{S}_{K}$ to be the set of infinite addresses $\underline{s}$ such that every prefix of length $n \geq N$ of $\underline{s}$ is an element of $\mathcal{S}_{n}$. In order to show that this set has the properties asserted in Theorem 8.3, we investigate the sets $\mathcal{S}_{n}(z)$ more closely.

Claim 1. The following hold for all $n \geq N$ and all $z \in K$.

(i) $\mathcal{S}_{N}(z) \neq \emptyset$.

(ii) The shift map $\sigma$ maps $\mathcal{S}_{n+1}(z)$ onto $\mathcal{S}_{n}(f(z))$.

(iii) $\mathcal{S}_{n}(z) \neq \emptyset$.

(iv) Suppose that $\underline{s}=F_{0} F_{1} \ldots F_{n} \in \mathcal{S}_{n+1}(z)$. Let $\pi_{n}(\underline{s})$ be the prefix of length $n$ of $\underline{s}$; i.e., $\pi_{n}(\underline{s})=F_{0} F_{1} \ldots F_{n-1}$. Then $\pi_{n}(\underline{s}) \in \mathcal{S}_{n}(z)$.

(v) In particular, $\pi_{N}\left(\sigma^{j}(\underline{s})\right) \in \mathcal{S}_{N}\left(f^{j}(z)\right)$ for $j=0, \ldots, n-N$.

Nb. In (ii), we would like to claim that $\sigma: \mathcal{S}_{n+1}(z) \rightarrow \mathcal{S}_{n}(f(z))$ is a bijection. However, it is conceivable that a tail $\tau$ of level $n$ intersects $B_{n-N}(f(z))$ in more than one connected component, and that $\tau \cup B_{n-N}(f(z))$ surrounds a singular value of $f$. In this situation, there may be two different components of $f^{-1}(\tau)$ that intersect $B_{n+1-N}(z)$, and $\sigma$ may therefore not be injective on $\mathcal{S}_{n+1}(z)$. Nonetheless, Claim 3 below implies that this situation can arise only for small $n$. Hence, for sufficiently large $n$, the map $\sigma: \mathcal{S}_{n+1}(z) \rightarrow \mathcal{S}_{n}(f(z))$ will turn out to be a bijection after all (see Claim 4). 
Proof The first claim holds by choice of $N_{1}$. Part (ii) is immediate from the fact that $f: B_{n+1-N}(z) \rightarrow B_{n-N}(f(z))$ is a conformal isomorphism. Claim (iii) follows from these first two claims by induction.

Now let us prove (iv). So suppose that $\underline{s} \in \mathcal{S}_{n+1}(z)$, and let $w \in \tau_{n+1}(\underline{s}) \cap$ $B_{n+1-N}(z)$. Recall from Lemma 6.10 that $\tau_{n+1}(\underline{s}) \subset \stackrel{\infty}{\tau}_{n+1}(\underline{s}) \cup V_{n}(\underline{s})$. First consider the case where $w \in \stackrel{\infty}{\tau}_{n+1}(\underline{s})$. Then $\underline{s} \in \mathcal{S}_{n}(z)$ since $w \in \stackrel{\infty}{\tau}_{n+1}(\underline{s}) \cap B_{n+1-N}(z) \subset$ $\tau_{n}(\underline{s}) \cap B_{n-N}(z)$.

Now suppose that $w \in V_{n}(\underline{s})$. By definition,

$$
\zeta_{n}(\underline{s}) \in V_{n}(\underline{s}) \cap \tau_{n}(\underline{s}) .
$$

Set $\underline{\tilde{s}}:=\sigma^{n-N}(\underline{s}), \tilde{z}:=f^{n-N}(z)$ and $\tilde{w}:=f^{n-N}(w)$. Then $\tilde{w} \in V_{N}(\underline{\tilde{s}}) \cap B_{1}(\tilde{z})$. By definition of $\varepsilon$ and choice of $N$, it follows that $V_{N}(\underline{\tilde{s}}) \subset B_{0}(\tilde{z})$. Now $B_{n-N}(z)$ is mapped conformally to $B_{0}(\tilde{z})$ by $f^{n-N}$. Since $V_{n}(\underline{s})$ is a connected component of $f^{N-n}\left(V_{N}(\underline{\tilde{s}})\right)$ and intersects $B_{n-N}(z)$, we see that $B_{n-N}(z) \supset V_{n}(\underline{s}) \ni \zeta_{n}(\underline{s})$. By $(10.2)$, we see that $\tau_{n}(\underline{s}) \cap B_{n-N}(z) \neq \emptyset$. This proves (iv)).

The final claim (v) follows by induction from (ii) and (iv).

Claim 2. There is a finite collection $\tilde{\mathcal{F}} \supset \mathcal{F}$ of fundamental domains such that all entries of addresses in $\bigcup_{n \geq N} \mathcal{S}_{n}$ are in $\tilde{\mathcal{F}}$.

Proof Let $\tilde{\mathcal{F}}$ be obtained by adding to $\mathcal{F}$ all fundamental domains appearing in the finitely many external addresses in $\mathcal{S}_{N}$. By part (v) of Claim 1, it follows that all entries of $\underline{s} \in \mathcal{S}_{n}$ belong to $\tilde{\mathcal{F}}$, for all $n \geq N$.

Applying Lemma 6.10 again, this time to the collection $\tilde{\mathcal{F}}$, we obtain a simplyconnected domain $\tilde{V}$ that we can use to study the accumulation sets of the addresses in $\mathcal{S}_{K}$.

Claim 3. For every $k \geq 0$ there is an $n_{0} \geq N$ with the following property. If $z \in K$, $n \geq n_{0}$ and $\underline{s} \in \mathcal{S}_{n}(z)$, then $\tilde{V}_{n}(\underline{s}) \subset B_{k}(z)$.

Proof By assumption, $K \subset D$. In particular, there exists $M>0$ such that no fundamental tail of level $N$ intersects the neighbourhood $U_{M}$ of $K$. Recall that $f\left(U_{j+1}\right)=U_{j}$ for all $j$, and that the image of a fundamental tail of level $j+1$ is a tail of level $j$. Hence it follows inductively that, if $n \geq N+M$, no fundamental tail of level $n-M$ intersects $U_{n-N}$.

Let $n \geq N+M$, let $z \in K$, and let $\underline{s} \in \mathcal{S}_{n}(z)$. Then

$$
\tau_{n}(\underline{s}) \subset \tau_{n-M}(\underline{s}) \cup \bigcup_{\tilde{n}=n-M}^{n-1} \tilde{V}_{\tilde{n}}(\underline{s})
$$

by Lemma $6.10(2)$. Observe that $\tau_{n}(\underline{s})$ intersects $B_{n-N}(z) \subset U_{n-N}$ by definition of $\mathcal{S}_{n}(z)$, while $\tau_{n-M}(\underline{s})$ is disjoint from $U_{n-N}$ by the above. Hence there exists some $\tilde{n} \in\{n-M, \ldots, n-1\}$ such that $\tilde{V}_{\tilde{n}}(\underline{s}) \cap B_{n-N}(z) \neq \emptyset$. 
Let $k \geq 0$. If $n_{1} \geq N$ is sufficiently large, then by Lemma 6.2,

$$
\operatorname{diam}\left(\tilde{V}_{n}(\underline{s})\right)<\varepsilon_{k}:=\frac{\min _{z \in K} \operatorname{dist}\left(B_{k+1}(z), \partial B_{k}(z)\right)}{M+1}
$$

whenever $n \geq n_{1}, \underline{s} \in \mathcal{S}_{n}(z)$ for some $z \in K$, and $\tilde{V}_{n}(\underline{s}) \cap U_{0} \neq \emptyset$. Set

$$
n_{0}:=\max \left(n_{1}+M, k+N+1\right) \geq N+M
$$

and suppose that $z, n$ and $\underline{s}$ are as in the statement of Claim 3.

Let $\tilde{n}$ be as above; i.e., $\tilde{n} \geq n-M \geq n_{1}$ is such that $\tilde{V}_{\tilde{n}}(\underline{s}) \cap B_{n-N}(z) \neq \emptyset$. Since $n-N \geq n_{0}-N \geq k+1$, we see that $\tilde{V}_{\tilde{n}}(\underline{s})$ intersects $B_{k+1}(z)$.

It follows inductively for $j=\tilde{n}, \tilde{n}+1, \ldots, n$ that, for all $\zeta \in \tilde{V}_{j}(\underline{s})$,

$$
\operatorname{dist}\left(\zeta, B_{k+1}(z)\right)<(j+1-\tilde{n}) \cdot \varepsilon_{k} \leq \operatorname{dist}\left(B_{k+1}(z), \partial B_{k}(z)\right) .
$$

(In the inductive step, we use that $\tilde{V}_{j}(\underline{s}) \cap \tilde{V}_{j-1}(\underline{s}) \neq \emptyset$ by Claim 2 and Lemma 6.10 (3), and that $j \geq n_{1}$.) In particular, $\tilde{V}_{n}(\underline{s}) \subset B_{k}(z)$, as required.

Claim 4. Let $n_{0}$ be as in Claim 3, for $k=1$. Then $\sigma: \mathcal{S}_{n+1}(z) \rightarrow \mathcal{S}_{n}(f(z))$ is a bijection for all $n \geq n_{0}$ and $z \in K$.

Proof By Claim 1 (ii), it remains to show that $\sigma: \mathcal{S}_{n+1}(z) \rightarrow \mathcal{S}_{n}(f(z))$ is injective. Let $z \in K$ and let $\underline{s}^{1}, \underline{s}^{2} \in \mathcal{S}_{n+1}(z)$ with $\sigma\left(\underline{s}^{1}\right)=\sigma\left(\underline{s}^{2}\right)=: \underline{\tilde{s}}$. Then, for $j=1,2$, we know that $\tilde{V}_{n+1}\left(\underline{s}^{j}\right) \subset B_{1}(z)$ by choice of $n_{0}$, and $\tilde{V}_{n}(\underline{\tilde{s}})=\bar{f}\left(\tilde{V}_{n+1}\left(\underline{s}^{j}\right)\right) \subset B_{0}(f(z))$. Since $f: B_{1}(z) \rightarrow B_{0}(f(z))$ is univalent, it follows that $\tilde{V}_{n+1}\left(\underline{s}^{1}\right)=\tilde{V}_{n+1}\left(\underline{s}^{2}\right)$, and hence $\underline{s}^{1}=\underline{s}^{2}$, as required.

Now consider the directed graph $G$ whose vertices are the elements of

$$
\mathcal{V}(G):=\bigcup_{n \geq N} \mathcal{S}_{n},
$$

and which contains an edge from $\pi_{n}(\underline{s})$ to $\underline{s}$ for every $\underline{s} \in \mathcal{S}_{n+1}$. Note that $G$ is a locally finite, infinite graph on countably many vertices. For $z \in K$, let $G_{z}$ be the induced subgraph of $G$ whose vertices are the elements of $\bigcup_{n>N} \mathcal{S}_{n}(z)$. By (iii) and (iv) of Claim 1, we can apply König's lemma, and $G_{z}$ contains an infinite path for every $z \in K$.

Recall that $\mathcal{S}_{K}$ is the set of infinite external addresses $\underline{s}$ such that $\pi_{n}(\underline{s}) \in \mathcal{S}_{n}$ for all $n \geq N$. If $\underline{s} \in \mathcal{S}_{K}$, the sequence $\left(\pi_{n}(\underline{s})\right)_{n=N}^{\infty}$ forms an infinite path in $G$. Conversely, every infinite path in $G$ determines an associated address $\underline{s} \in \mathcal{S}_{K}$. For $z \in K$, denote by $\mathcal{S}_{z}$ the set of all $\underline{s} \in \mathcal{S}_{K}$ with $\pi_{n}(z) \in \mathcal{S}_{n}(z)$ for all $n \geq N$. By the above, $\mathcal{S}_{z} \neq \emptyset$ for all $z \in K$.

The set $\mathcal{S}_{K}$ is shift-invariant by part (ii) of Claim 1. Furthermore, $\mathcal{S}_{K}$ is contained in the compact set of addresses all of whose entries are taken from $\tilde{\mathcal{F}}$; we need to show that $\mathcal{S}_{K}$ is itself compact. Suppose that $\left(\underline{s}^{k}\right)_{k=0}^{\infty}$ is a sequence of addresses in $\mathcal{S}_{K}$ converging to some address $\underline{s}$. Then the prefixes $\pi_{n+1}(\underline{s})$ and $\pi_{n+1}\left(\underline{s}^{k}\right)$ agree for 
all sufficiently large $k$, and in particular $\pi_{n}(\underline{s})$ and $\pi_{n+1}(\underline{s})$ are two vertices of $G$ connected by an edge. It follows that $\underline{s}$ is indeed represented by an infinite path in $G$, and hence $\underline{s} \in \mathcal{S}_{K}$ as required.

To prove claim (a) of Theorem 8.3, we must show that $G_{\underline{s}}$ lands at a point $z_{0}(\underline{s}) \in K$ for all $\underline{s} \in \mathcal{S}_{K}$. By Claim 3 , there is some $n_{0}$ such that, for all $n \geq n_{0}$ and all $\underline{s} \in \mathcal{S}_{K}$, there is $z \in K$ such that $\tilde{V}_{n}(\underline{s}) \subset B_{0}(z)$. In particular, diam $\tilde{V}_{n_{0}}(\underline{s}) \leq 2 \delta$, and by expansion of $f$ on $U$, we conclude that

$$
\operatorname{diam} \tilde{V}_{n}(\underline{s}) \leq 2^{n_{0}-n+1} \cdot \delta .
$$

In particular, $\zeta_{n}(\underline{s})$ is a Cauchy sequence, and hence convergent. So $G_{\underline{s}}$ lands at a point $z_{0}(\underline{s})$ with

$$
\operatorname{dist}\left(z_{0}(\underline{s}), \tilde{V}_{n}(\underline{s})\right) \leq 2^{n_{0}-n+1} \cdot \delta,
$$

for all $n \geq n_{0}$. It is clear from Claim 3 that the landing point $z_{0}(\underline{s})$ belongs to $K$. Furthermore, if $z \in K$ and $\underline{s} \in \mathcal{S}_{z}$, then $z_{0}(\underline{s})=z$ by Claim 3 .

As $\mathcal{S}_{z} \neq \emptyset$ for all $z \in K$, this shows that the function $\underline{s} \mapsto z_{0}(\underline{s})$ is surjective. To prove continuity, suppose that $\underline{s}, \underline{\tilde{s}} \in \mathcal{S}_{K}$ agree in the first $n \geq n_{0}$ entries. Then $\tilde{V}_{n}(\underline{s})=\tilde{V}_{n}(\underline{\tilde{s}})$, and hence

$$
\operatorname{dist}\left(z_{0}(\underline{s}), z_{0}(\underline{\tilde{s}})\right) \leq 3 \cdot 2^{n_{0}-n+1} \cdot \delta
$$

by (10.3) and (10.4). This completes the proof of (b).

Part (d) follows directly from Claim 3. It remains to establish (c).

So let $z \in K$ be a periodic point of period $p$. By Lemma 9.2 and (a), it is enough to show that $\mathcal{S}_{z}$ contains a periodic address. Let $n_{0}$ be as in Claim 4 ; by increasing $n_{0}$ if necessary, we can assume that $p$ divides $n_{0}$. Let $\psi: \mathcal{S}_{n_{0}}(z) \rightarrow \mathcal{S}_{2 n_{0}}(z)$ be the inverse of $\left.\sigma^{n_{0}}\right|_{\mathcal{S}_{2 n_{0}}(z)}$. Since $\mathcal{S}_{n_{0}}(z)$ is finite, the function $\varphi:=\pi_{n_{0}} \circ \psi$ has a periodic element; say $\varphi^{k_{0}}(\underline{s})=\underline{s}$. For $k \geq 0$, let $\underline{s}^{k}$ be the unique preimage of $\underline{s}$ under $\sigma^{k n_{0}}$ in $\mathcal{S}_{(k+1) n_{0}}(z)$. We claim that

$$
\pi_{n_{0}}\left(\underline{s}^{k+1}\right)=\varphi\left(\pi_{n_{0}}\left(\underline{s}^{k}\right)\right)
$$

for all $k \geq 0$. This is true for $k=0$ by definition. If $k>0$, we have

$$
\pi_{2 n_{0}}\left(\underline{s}^{k+1}\right)=\psi\left(\sigma^{n_{0}}\left(\pi_{2 n_{0}}\left(\underline{s}^{k+1}\right)\right)\right)=\psi\left(\pi_{n_{0}}\left(\sigma^{n_{0}}\left(\underline{s}^{k+1}\right)\right)\right)=\psi\left(\pi_{n_{0}}\left(\underline{s}^{k}\right)\right) .
$$

Hence

$$
\pi_{n_{0}}\left(\underline{s}^{k+1}\right)=\pi_{n_{0}}\left(\psi\left(\pi_{n_{0}}\left(\underline{s}^{k}\right)\right)\right)=\varphi\left(\pi_{n_{0}}\left(\underline{s}^{k}\right)\right) .
$$

So $\pi_{n_{0}}\left(\underline{s}^{k}\right)=\varphi^{k}(\underline{s})$ for all $k \geq 0$. Hence $\underline{s}^{k}$ can be written as a concatenation

$$
\underline{s}^{k}=\varphi^{k}(\underline{s}) \underline{s}^{k_{1}}=\cdots=\varphi^{k}(\underline{s}) \varphi^{k-1}(\underline{s}) \ldots \varphi^{1}(\underline{s}) \underline{s} .
$$

Since $\underline{s}$ is periodic under $\varphi$, of period $k_{0}$, we conclude that $\underline{s}^{k}=\pi_{(k+1) n_{0}}\left(\underline{s}^{k+k_{0}}\right)$. Hence there is an infinite path in $G_{z}$ passing through the vertices $\underline{s}^{j \cdot k_{0}}, j \geq 0$. The 
associated address is the periodic sequence $\left(\varphi^{k_{0}-1}(\underline{s}) \ldots \varphi^{1}(\underline{s}) \underline{s}\right)^{\infty}$, and the proof is complete.

We note the following corollary, which proves the accessibility of certain singular values. For definitions, we refer to [RvS11].

COROLlary 10.1 (Accessibility of non-recurrent singular values). Let $f$ be a postsingularly bounded transcendental entire function, and let $v \in J(f)$ be a non-recurrent singular value for $f$ whose forward orbit does not pass through any critical points. Suppose that the $\omega$-limit set of $v$ does not contain parabolic points, and does not intersect the $\omega$-limit set of a recurrent critical point or of a singular value contained in a wandering domain. Then there is a bounded-address dreadlock of $f$ that lands at $v$.

Proof By [RvS11, Theorem 1.2], if the postsingular set is bounded then any forward invariant compact subset of the Julia set is hyperbolic provided it does not contain parabolic points, critical points, or it intersects the $\omega$-limit set of a critical point or of a singular value contained in wandering domains. Hence $P(a):=\overline{\bigcup_{n} f^{n}(a)}$ is hyperbolic and every point in $P(a)$ is the landing point of a dreadlock.

\section{Landing at Parabolic Points}

We now complete the proof of our analogue of the Douady-Hubbard landing theorem, Theorem 8.1 , by showing that parabolic periodic points are also accessible by dreadlocks.

Theorem 11.1 (Parabolic points are accessible by dreadlocks). Let $f \in \mathcal{B}$ with bounded postsingular set, and let $z_{0}$ be a parabolic periodic point. Then there is a periodic dreadlock of $f$ that lands at $z_{0}$.

Let $f$ be as in the statement of the theorem. By passing to an iterate, we may assume that $f^{\prime}\left(z_{0}\right)=1$. So $z_{0}$ is a multiple fixed point of $f$, say of multiplicity $m+1$ for $f$. Then there are $m$ unit vectors $v_{1} \ldots v_{n}$, called repelling directions at $z_{0}$. Any backward orbit of $f$ converging to $z_{0}$ must asymptotically converge to $z_{0}$ along one of these directions; see [Mil06, Lemma 10.1]. Similarly, there are $n$ attracting directions $w_{n}$ such that any forward orbit $\left(f^{n}(z)\right)_{n=0}^{\infty}$ converging to $z_{0}$ must converge to $z_{0}$ along one of these attracting directions $w_{n}$.

Let $U$ be a small simply connected neighborhood of $z_{0}$ on which $f$ is univalent, and let $\psi: f(U) \rightarrow U$ be the branch of $f^{-1}$ that fixes $z_{0}$. A petal for an attracting (resp. repelling) direction $w$ (resp. $v$ ) is an open set $P \subset U$ containing $z_{0}$ on its boundary, such that

(1) $f(P) \subset P($ resp. $\psi(P) \subset P)$;

(2) an orbit $z \rightarrow f(z) \rightarrow \ldots$ (resp. $z \rightarrow \psi(z) \rightarrow \ldots$ ) is eventually absorbed by $P$ if and only if it converges to $z_{0}$ from the direction $w$ (resp. $v$ ). 
Petals for a given repelling or attracting direction are far from unique. For each repelling direction $v$, we can choose a repelling petal $P_{v}$ for $v$ which is simply connected, and such that $\overline{\psi\left(P_{v}\right)} \subset P_{v} \cup\left\{z_{0}\right\}$ and $\left.\psi^{n}\right|_{P_{v}} \rightarrow z_{0}$ uniformly on $P_{v}$. Similarly, for each attracting direction $w$ we choose a simply connected attracting petal $P_{w}$ such that $f^{n} \rightarrow z_{0}$ uniformly on $P_{w}$. We furthermore require that the union of these $n$ attracting and $n$ repelling petals forms a punctured neighborhood of $z_{0}$ (see Definition 10.6 and Theorem 10.7 in [Mil06] and the subsequent discussion).

Definition 11.2 (Landing of dreadlocks along a repelling direction). Let $\zeta \in W_{0}$, let $G_{\underline{s}}$ be a periodic dreadlock of $f$, and let $v$ be a repelling direction at $z_{0}$. We say that $G_{\underline{s}}$ lands at $z_{0}$ along $v$ if the backwards orbit $\left(\zeta_{n}(\underline{s})\right)_{n=1}^{\infty}$ converges to $z_{0}$ along the direction $v$.

We remark that it is not difficult to see that this is equivalent to requiring that $V_{n}(\underline{s}) \subset P_{v}$ for all sufficiently large $n$, where $V$ is as in Lemma 6.10. In particular, the definition is independent of the choice of the base point $\zeta$.

The following establishes Theorem 11.1.

Proposition 11.3 (Accessibility along repelling directions). Let $v$ be a repelling direction of $f$ at $z_{0}$. Then there is at least one periodic dreadlock landing at $z_{0}$ along $v$.

Proof Let $\zeta \in W_{0}$ and let $V$ be as in Lemma 6.10, with $\mathcal{F}$ once again the finite collection of fundamental domains whose closure intersects $\bar{D}$. Since $\bar{V} \subset \mathbb{C} \backslash \mathcal{P}(f)$ and $z_{0} \in \mathcal{P}(f) \subset D$, we may assume that the repelling petals $P_{v}$ and attracting petals $P_{w}$ chosen above all have closures disjoint from $V \cup W_{0}$.

Let us define $B_{i}:=\psi^{i}\left(P_{v}\right)$ for $i \geq 0$. Let $\mathcal{A}$ be the union of the attracting petals $P_{w}$. Since the union of attracting and repelling petals is a punctured neighbourhood of $z_{0}$, all points of $\partial B_{0}$ that are sufficiently close to $z_{0}$ must lie in $\mathcal{A} \cup\left\{z_{0}\right\}$. So $\partial B_{0} \backslash\left(\mathcal{A} \cup\left\{z_{0}\right\}\right)$ is a compact set disjoint from $\overline{B_{1}}$, and

$$
\varepsilon:=\operatorname{dist}\left(\left(\partial B_{0}\right) \backslash\left(\mathcal{A} \cup\left\{z_{0}\right\}\right), \overline{B_{1}}\right)>0 .
$$

Since $B_{1}$ intersects $J(f)$, there is an $N_{1}$ such that $f^{-n}(\zeta) \cap B_{1} \neq \emptyset$ for $n \geq$ $N_{1}$. In particular, there exists some finite external address of length $n$ such that $\tau_{n}(\underline{s}) \cap B_{1} \neq \emptyset$. By Lemma 6.2, there is $N \geq N_{1}$ such that, for all $n \geq N$ and all infinite external addresses $\underline{s}$ with $V_{n}(\underline{s}) \cap \overline{B_{0}} \neq \emptyset$, diam $V_{n}(\underline{s})<\varepsilon$ whenever $n \geq N$. Observe that $V_{n}(\underline{s}) \cap \mathcal{A}=\emptyset$ by our choice of petals. In particular, if $n \geq N$ and $V_{n}(\underline{s}) \cap B_{1} \neq \emptyset$, then $V_{n}(\underline{s}) \cap \partial B_{0}=\emptyset$, and hence $V_{n}(\underline{s}) \subset B_{0}$.

As in the proof of Theorem 8.3, for $n \geq N$ we define $\mathcal{S}_{n}$ to consist of those finite external addresses of length $n$ for which $\tau_{n}(\underline{s})$ intersects $B_{n-N}$. The remainder of the proof then proceeds analogously.

In the case that all periodic dreadlocks are hairs (for example, if $f$ is criniferous), our Proposition 11.3 is a corollary of the Main Theorem in [BF15] (since the hypothesis that periodic rays land is implied by assuming bounded postsingular set), 
with a completely different proof. We remark that it is plausible that the results of [BF15] can also be extended to non-criniferous functions, using dreadlocks instead of hairs.

Proof of Theorem 8.1 That every periodic dreadlock lands at a repelling or parabolic point was proved in Theorem 9.1. Let $z_{0}$ be a repelling or parabolic point. If $z_{0}$ is repelling, then the orbit of $z_{0}$ is a hyperbolic set, and it follows from Theorem 8.3 that $z_{0}$ is the landing point of a periodic dreadlock. If $z_{0}$ is a parabolic point, then this fact follows from Theorem 11.1. By Lemma 9.2 there are only finitely many dreadlocks landing at $z_{0}$ and they are all periodic of the same period.

\section{Dreadlocks Landing Together at Points in a Hyperbolic Set}

Recall from Theorem 8.1 that, for a repelling periodic point $z_{0}$ of a postsingularly bounded function $f$, the number of dreadlocks landing at $z_{0}$ is finite. In the polynomial case, this holds also for every point $z_{0}$ in a hyperbolic set $K$ of $f$. It is plausible that this remains true also in the transcendental entire case. For postsingularly bounded exponential maps, the claim is proved in [BL14, Proposition 4.5], where it is proved that the number of hairs in question is even uniformly bounded (depending on $K$ ). However, the proof uses the fact that postsingularly bounded exponential maps are non-recurrent, and hence the postsingular set is itself a hyperbolic set.

Here we shall be content with proving that the number of dreadlocks of a postsingularly bounded function $f$ landing at a given point of a hyperbolic set is (pointwise) finite, in the important special case where $f$ belongs to the Speiser class; i.e., the set of singular values $S(f)$ is finite.

Theorem 12.1 (Finitely many dreadlocks landing together). Let $f$ be a postsingularly bounded entire function with finitely many singular values. Suppose that $z_{0} \in J(f) \backslash I(f)$ is neither a Cremer periodic point nor a preimage of such. Then the number of bounded-address dreadlocks $G_{\underline{s}}$ landing at $z_{0}$ is finite.

REMARK 1. The assumption that $f$ is postsingularly bounded implies, via Theorem 8.1 and Lemma 9.2, that one can restrict to the case where $z_{0}$ is not (pre-)periodic. In addition, this hypothesis and the restriction to bounded addresses $\underline{s}$ ensure that we can speak about the dreadlocks $G_{\underline{s}}$ and their landing properties at all. (Recall Remark 9.3.) However, the argument can be applied also in more general circumstances. For example, the same proof can be used to show the following: if $S(f)$ is finite (but the postsingular set is not necessarily bounded), and $z_{0} \in J(f) \backslash I(f)$ is not periodic and also is the landing point of at least one boundedaddress hair, then the number of hairs landing at $z_{0}$ is finite, and all of them have bounded addresses.

REMARK 2. The assumption that $z_{0} \notin I(f)$ is made to avoid complications in the case where $z_{0}$ itself belongs to one of the dreadlocks landing at $z_{0}$. An escaping 
point in a bounded-address dreadlock cannot in fact be accessible by the same or another dreadlock, due to the presence of other dreadlocks accumulating on it from both sides; recall the proof of Corollary 6.7, and compare [Rem16, Theorem 2.3]. Assuming this fact, the assumption that $z_{0} \notin I(f)$ could be omitted.

COROLlary 12.2 (Finiteness of dreadlock portraits at hyperbolic sets). Let $f$ be a postsingularly bounded entire function with finitely many singular values. If $K$ is a hyperbolic set for $f$, then every point $z_{0} \in K$ is the landing point of at least one and at most finitely many bounded-address dreadlocks.

Proof By definition, a hyperbolic set contains no Cremer periodic points, their preimages, or escaping points. Hence this is a combination of Theorems 8.2 and 12.1.

We now fix a postsingularly bounded entire function $f$ with $\# S(f)<\infty$ for the remainder of the section. The key property that we need to establish in the proof of Theorem 12.1 is that the addresses of dreadlocks landing at $z_{0}$ are uniformly bounded, in the sense that they all take their entries from a common finite family of fundamental domains. This is the content of the following lemma.

LEMMA 12.3 Let $\mathcal{F}_{1}$ be a finite collection of fundamental domains for $f$. Then there exists another finite collection $\mathcal{F}_{2} \supset \mathcal{F}_{1}$ of fundamental domains such that the following holds. Suppose that $\underline{s}^{1}$ takes only entries from $\mathcal{F}_{1}$ and that $G_{\underline{s}^{1}}$ lands at a non-escaping point $z_{0} \in \mathbb{C}$. If $\underline{s}^{2}$ is bounded and $G_{\underline{s}^{2}}$ also lands at $z_{0}$, then all entries of $\underline{s}^{2}$ belong to $\mathcal{F}_{2}$.

Let us suppose for a moment that the function $f$ is criniferous. Then the idea of the proof of Lemma 12.3 can be described as follows. If $G_{\underline{s}^{1}}$ and $G_{\underline{s}^{2}}$ land together at a point $z_{0}$, the dreadlocks $G_{\sigma\left(\underline{s}^{1}\right)}$ and $G_{\sigma\left(\underline{s}^{2}\right)}$ also land together at $f\left(z_{0}\right)$, by continuity of $f$. There is a branch $\varphi$ of the inverse of $f$ on the hair $G_{\sigma\left(\underline{s}^{1}\right)}$ that maps it to $G_{\underline{s}^{1}}$. The curve $G_{\underline{s}^{1}} \cup\left\{z_{0}\right\} \cup G_{\underline{s}^{2}}$ is then obtained by analytic continuation of $\varphi$ along the image curve $G_{\sigma\left(\underline{s}^{1}\right)} \cup\left\{f\left(z_{0}\right)\right\} \cup G_{\sigma\left(\underline{s}^{2}\right)}$. For this reason, the homotopy class of the latter curve in $\mathbb{C} \backslash S(f)$, together with the first entry of $\underline{s}^{1}$, essentially determines the first entry of $\underline{s}^{2}$. As different pairs of hairs landing at the same point are disjoint, and $S(f)$ is finite, there are only finitely many possible such homotopy classes. The claim follows. In order to make this argument precise in the general case, i.e. where the dreadlocks are not necessarily hairs, we should clarify what we mean by "homotopy classes". Let us fix the postsingularly bounded function $f$ with finite singular set for the remainder of the section.

Let $\Gamma$ be the class of continuous curves $\gamma: \mathbb{R} \rightarrow \mathbb{C} \backslash S(f)$ that tend to infinity within $W_{0}$ in both directions. We shall say that such curves $\gamma_{1}$ and $\gamma_{2}$ are homotopic (in $\Gamma$ ) if they are homotopic (relative to their endpoints at infinity) in $\mathbb{C} \backslash(S(f) \cup \tilde{\delta}$ ), for some infinite piece $\tilde{\delta}$ of the curve $\delta$ used in the definition of fundamental domains.

Similarly, let $\tilde{\Gamma}$ denote the set of curves connecting a finite endpoint $z_{0} \in \mathbb{C}$ (possibly belonging to $S(f)$ ) to infinity within $\mathbb{C} \backslash S(f)$, again tending to infinity 
within $W_{0}$. Then we analogously define homotopy classes for curves in $\tilde{\Gamma}$ having the same endpoint.

We can now introduce a convenient notion for homotopy classes of boundedaddress dreadlocks. Suppose that $\underline{s}$ is a bounded external address, and that the dreadlock $G_{\underline{s}}$ lands at a point $z_{0} \in \mathbb{C} \backslash G_{\underline{s}}$. Then there is an infinite piece $\tilde{\delta}$ of $\delta$ not intersecting $G_{s}$. It follows that there is a Jordan curve $J$, passing through infinity, that separates $\overline{G_{\underline{s}}}$ from $\tilde{\delta}$ and all of the finitely many points of $S(f) \backslash\left\{z_{0}\right\}$. Let $\gamma$ be an arc connecting $z_{0}$ to infinity in the connected component $V$ of $\mathbb{C} \backslash J$ containing $z_{0}$. The homotopy class of $G_{\underline{s}}$ is the homotopy class of $\gamma$ in $\tilde{\Gamma}$, as defined above.

Note that this homotopy class depends only on $\underline{s}$. Indeed, suppose that $\tilde{V}$ is a second domain as above, and $\tilde{\gamma} \subset \tilde{V}$ connects $z_{0}$ to infinity. Since $\overline{G_{\underline{s}}} \subset V \cap \tilde{V}=: U$, this open set $U$ contains a curve $\alpha$ connecting $z_{0}$ to infinity. See Lemma A.1 of [Rem08]. Since $V$ is simply-connected, $\alpha$ is homotopic to $\gamma$ in $V$, and hence in $\tilde{\Gamma}$. For the same reason, $\alpha$ is homotopic to $\tilde{\gamma}$.

Observation 12.4 (Disjoint curves representing homotopy classes). Let $\mathcal{S}_{1}, \ldots, \mathcal{S}_{n}$ be finitely many different bounded external addresses, such that each $G_{\mathcal{S}_{j}}$ lands at a non-escaping point $z_{j} \in \mathbb{C}$ for all $j$. (We do not assume that all $z_{j}$ are distinct.) Then there exists a collection $\left(\gamma_{j}\right)_{j=1}^{n}$ of arcs to infinity, with $\gamma_{j}$ in the homotopy class of $G_{\underline{s}}$, such that these arcs are pairwise disjoint apart from common endpoints.

Proof Similarly as above, we can find a finite collection of Jordan curves $\left(J_{\ell}\right)_{\ell=1}^{m}$, disjoint from $\tilde{\delta} \cup \bigcup_{j=1}^{n} \overline{G_{\mathcal{S}_{j}}}$, such that any two distinct landing points $z_{j_{1}}$ and $z_{j_{2}}$ are separated by some $J_{\ell}$. (Here, as above, $\tilde{\delta}$ is an infinite piece of the curve $\delta$ that does not intersect any of the dreadlocks under consideration.)

Let $V_{j}$ be the connected component of $\mathbb{C} \backslash \bigcup_{\ell=1}^{m} J_{\ell}$ containing $z_{j}$. We can choose the curve $\Gamma$ in the definition of the homotopy class of $G_{\mathcal{S}_{j}}$ in such a way that $\Gamma$ additionally separates $z_{j}$ from $\partial V_{j}$. This shows that the $\gamma_{j}$ may be chosen disjoint, except possibly for those having a common endpoint. But any curves with a common endpoint belong to the same $V_{j}$, and therefore can also be moved by homotopy within the simply-connected domain $V_{j}$ to be disjoint, except at that endpoint. This completes the proof.

If two bounded-address dreadlocks $G_{\underline{s}^{1}}$ and $G_{\underline{s}^{2}}$ land at a common non-escaping point $z_{0}$, we shall refer to these two dreadlocks as a dreadlock pair. If $z_{0} \notin S(f)$, then we can form a curve in $\Gamma$ by combining two arcs $\gamma_{1}$ and $\gamma_{2}$, in the homotopy class of $G_{\underline{s}^{1}}$ and $G_{\underline{s}^{2}}$, respectively. The corresponding homotopy class is called the homotopy class of the dreadlock pair.

LEMma 12.5 (Finitely many homotopy classes). There are only finitely many different homotopy classes of dreadlock pairs not landing at singular values.

Similarly, for any $z_{0} \in \mathbb{C}$, there are only finitely many homotopy classes of dreadlocks landing at $z_{0}$. 
Proof The curves representing the homotopy class of two different dreadlock pairs are disjoint, except for the endpoints at infinity, and possibly a single additional point (if the dreadlock pairs land at the same point). Also recall that neither curve self-intersects. It follows that, if both curves wind around the same collection of singular values in positive orientation, and both either surround or do not surround an infinite piece of $\delta$, they represent the same homotopy class. As there are only finitely many singular values, the set of homotopy classes is finite.

The second claim follows in the same manner.

The following is immediate from the homotopy lifiting property.

Observation 12.6 (Connecting fundamental domains). Let $\gamma \in \Gamma$. Suppose that $F$ is a fundamental domain, and let $\tilde{\gamma}:(-\infty, \infty) \rightarrow \mathbb{C} \backslash f^{-1}(S(f))$ be the unique lift of $\gamma$ under $f$ such that $\tilde{\gamma}(-t) \in F$ for all sufficiently large $t$. Then there is a fundamental domain $\tilde{F}$ such that $\tilde{\gamma}(t) \in \tilde{F}$ for large $t$, and $\tilde{F}$ depends only on $F$ and the homotopy class of $\gamma$ in $\Gamma$.

Similarly, let $\gamma \in \tilde{\Gamma}$ connect a finite point $z_{0} \in \mathbb{C}$ to $\infty$. If $F$ is a fundamental domain, and $\tilde{\gamma}$ is the lift of $\gamma$ under $f$ that tends to infinity within $F$, then the finite endpoint $w_{0}$ of $\tilde{\gamma}$ depends only on $F$ and the homotopy class of $\gamma$ in $\tilde{\Gamma}$.

Proof of Lemma 12.3 Let $\tilde{\mathcal{F}}_{2}$ consist of all domains $\tilde{F}$ as in Observation 12.6, where $F$ ranges over the finitely many elements of $\mathcal{F}_{1}$, and the homotopy class of $\gamma$ ranges over the finitely many homotopy classes of dreadlock pairs of $f$.

Now suppose that $G_{\underline{s}^{1}}$ and $G_{\underline{s}^{2}}$ form a dreadlock pair, with $F_{0}^{1} \in \mathcal{F}_{1}$. Let $z_{0}$ be the common landing point of the two dreadlocks. If $f\left(z_{0}\right) \notin S(f)$, then it follows from Observation 12.6 (applied to the curve $\left.\gamma_{\sigma\left(\underline{s}^{1}\right)} \cup\left\{f\left(z_{0}\right)\right\} \cup \gamma_{\sigma\left(\underline{s}^{2}\right)}\right)$ that $F_{0}^{2} \in \tilde{\mathcal{F}}_{2}$.

On the other hand, suppose that $s=f\left(z_{0}\right) \in S(f)$. Then, by Observation 12.6, $z_{0}$ depends only on the homotopy class of $\gamma_{\sigma\left(\underline{s}^{1}\right)}$, and the entry $F_{0}^{1}$. Hence, for each singular value $s$, there are only finitely many possible preimages $z_{0}$ that can arise as landing points of dreadlocks whose first entry is in $\mathcal{F}_{1}$.

Consider such $z_{0}$, and the curve $\gamma=\gamma_{\sigma\left(\underline{s}^{2}\right)} \in \tilde{\Gamma}$ connecting $f\left(z_{0}\right)$ to $\infty$. Then $\gamma$ has $d$ different lifts starting at $z_{0}$, where $d$ is the local degree of $f$ at $z_{0}$, tending to infinity within fundamental domains $\tilde{F}_{1}, \ldots, \tilde{F}_{d}$. This collection of fundamental domains depends only on the homotopy class of $\gamma$ by Observation 12.6. In particular, there is a collection $\mathcal{F}\left(z_{0}\right)$ of at most $m \cdot d$ fundamental domains, where $m$ is the (finite) number of homotopy classes of dreadlocks connecting $s$ to $\infty$, such that $F_{0}^{2} \in \mathcal{F}\left(z_{0}\right)$ whenever $\underline{s}^{2}$ is as above.

Recall that there are only finitely many singular values $s$, and for each of these only finitely many preimages $z_{0}$ as above. Thus we can add the finitely many sets $\mathcal{F}\left(z_{0}\right)$ to $\tilde{\mathcal{F}}_{2}$ to obtain a set $\mathcal{F}_{2}$ with the desired property.

Proof of Theorem 12.1 If $z_{0}$ is (pre-)periodic, then by assumption $f^{n}\left(z_{0}\right)$ is a repelling or parabolic periodic point for some $n \geq 0$. As remarked above, in this case the conclusion of the theorem holds by Theorem 8.1 and Lemma 9.2. Hence we can assume that $z_{0}$ is not a pre-periodic point. 
Since $f$ is postsingularly bounded, every orbit of $f$ passes through only finitely many critical points. Indeed, points with unbounded orbits cannot go through critical points at all, and the intersection of any bounded orbit with the (discrete) set of critical points is finite. Hence, passing to a forward iterate, we may additionally assume that the forward orbit of $z_{0}$ does not contain a critical point. Let $\mathcal{F}_{1}$ be the set of fundamental domains occurring in $\underline{s}$, and let $\mathcal{F}_{2}$ be the set whose existence is guaranteed by Lemma 12.3 ; say $\mathcal{F}_{2}=\left\{F^{0}, F^{1}, \ldots, F^{m-1}\right\}$, where we assume that

$$
F^{0} \prec F^{1} \prec \cdots \prec F^{m-1} \prec F^{1}
$$

with respect to the cyclical order at infinity.

Let $X$ be the set of points on the unit circle $S^{1}=\mathbb{R} / \mathbb{Z}$ having an $(m+1)$-ary expansion that contains only the entries $0, \ldots, m-1$. Via the $(m+1)$-ary expansion, this set is order-isomorphic to $\{0, \ldots, m-1\}^{\mathbb{N}}$, which in turn is clearly order-isomorphic to $\mathcal{F}_{2}^{\mathbb{N}}$. Let $\varphi: \mathcal{F}_{2}^{\mathbb{N}} \rightarrow X$ be this order-isomorphism; then $\varphi$ conjugates the shift on $\mathcal{F}_{2}^{\mathbb{N}}$ to the $(m+1)$-tupling map on $X$.

Suppose that $T_{0}$ is a collection of $p \geq 1$ bounded external addresses that land at $z_{0}$; we claim that $p \leq m+1$. Indeed, for $j \geq 0$, define $T_{j}:=\sigma^{j}\left(T_{0}\right)$. Then all dreadlocks at addresses in $T_{j}$ land at $f^{j}\left(z_{0}\right)$. Since $z_{0}$ is not pre-periodic and its orbit does not pass through any critical points, the $T_{j}$ are pairwise disjoint, and $\sigma: T_{j} \rightarrow T_{j+1}$ is an order-preserving bijection for all $j$. Furthermore, the $T_{j}$ are pairwise unlinked. That is, if $j \neq \tilde{j}$, then all elements of $T_{j}$ lie between the same two adjacent elements of $T_{\tilde{j}}$ with respect to circular order.

This means that the set $\varphi\left(T_{0}\right)$ is a wandering $p$-gon for the $(m+1)$-tupling map on $S^{1}$. Kiwi [Kiw02, Theorem 1.1] proved that polynomials of degree $d$ do not have wandering $(d+1)$-gons. A combinatorial version of this result (see [BL02, Theorem B]) implies that $p \leq m+1$ as claimed.

REMARK It seems likely that one can also directly prove the absence of wandering $d+2$-gons for maps with at most $d$ singular values. (Compare [AR17] for the proof of the case $d=1$, i.e. the no wandering triangles theorem for exponential maps.) This would imply that the number of dreadlocks in Theorem 12.1 is always bounded by $d+1$ (assuming that $z_{0}$ is not pre-critical).

\section{Appendix: Cyclic Order of Unbounded Closed Connected Sets}

In this section, suppose that $\mathcal{A}$ is any pairwise disjoint collection of unbounded, closed, connected subsets of $\mathbb{C}$ such that, for every $A \in \mathcal{A}$, all elements of $\mathcal{A} \backslash\{A\}$ belong to the same connected component of $\mathbb{C} \backslash A$. Observe that the latter condition holds, in particular, if no $A \in \mathcal{A}$ separates the plane.

The purpose of this section is to note that there is a natural cyclic order (at $\infty)$ on $A$. Recall that a cyclic order is a ternary relation $A \prec B \prec C$ that is cyclic, asymmetric, transitive and total [Čec69, § 5]. 
In our case, the relation $A \prec B \prec C$ means that $B$ lies between $A$ and $C$ in positive orientation. To make this precise, let us begin by defining a circular order on any finite subset of $\mathcal{A}$. So suppose that $A_{1}, \ldots, A_{n}(n \geq 3)$ are distinct elements of $\mathcal{A}$. Let $W_{j}$ be the connected component of $\mathbb{C} \backslash A_{j}$ that contains $A_{i}$ for $i \neq j$, and set $\tilde{A}_{j}:=\hat{\mathbb{C}} \backslash W_{j}$. Then $K:=\bigcup_{j=1}^{n} \tilde{A}_{j}$ is a compact, connected and full set in $\hat{\mathbb{C}}$, and its complement is

$$
W:=\hat{\mathbb{C}} \backslash K=\bigcap_{j=1}^{\infty} W_{j}
$$

In other words, the simply-connected domain $W$ is the unique connected component $W$ of $\mathbb{C} \backslash \bigcup_{j=1}^{n} A_{j}$ whose boundary intersects $A_{j}$ for each $j$.

We now consider the space of prime ends of $W$; see [Pom92, Section 2.4]. Recall that these form a topological circle, and therefore possess a natural cyclic order. Note that the connected components of $K \backslash\{\infty\}$ are precisely the $\tilde{A}_{j} \backslash\{\infty\}$. It follows (e.g. as a consequence of the plane separation theorem [Why42, Theorem 3.1, Chapter VI]) that there are exactly $n$ different accesses $\zeta_{1}, \ldots, \zeta_{n}$ to $\infty$ from $W$. They separate the circle of prime ends into $n$ complementary intervals $I_{1}, \ldots, I_{n}$, which may be labeled such that $I_{j}$ consists of those prime ends that can be represented by a sequence of cross-cuts both of whose endpoints belong to $A_{j}$. We define the circular order of the sets $A_{j}$ at $\infty$ (in positive orientation) to be the circular order of these intervals, taken in negative orientation.

If we add a new element $A_{n+1}$ of $\mathcal{A}$ to our collection, then it is easy to check that this does not change the definition of the circular order of $A_{1}, \ldots, A_{n}$. Hence we do indeed obtain a well-defined circular order on all of $\mathcal{A}$. Moreover, suppose that $\tilde{\mathcal{A}}$ is a second collection as above, where every element of $\tilde{\mathcal{A}}$ is contained in an element of $\mathcal{A}$ and every element of $\mathcal{A}$ contains exactly one element of $\tilde{\mathcal{A}}$. Then the cyclic order on $\tilde{\mathcal{A}}$ coincides with the corresponding order on $\mathcal{A}$.

We can use this observation to define cyclic order also for pairwise disjoint collections of open unbounded domains, each of which contains exactly one homotopy class of curves to infinity. (Simply replace each domain by a representative in the mentioned homotopy class.)

Furthermore, suppose that $U$ and $\tilde{U}$ are unbounded domains in $\mathbb{C}$, that $\varphi: U \rightarrow \tilde{U}$ is a conformal isomorphism. Also suppose that $\mathcal{A}$ and $\tilde{\mathcal{A}}$ are collections as above, whose elements are contained in $U$ and $\tilde{U}$, respectively, that $\varphi$ maps every element of $\mathcal{A}$ to an element of $\tilde{\mathcal{A}}$, and that all elements of $\tilde{\mathcal{A}}$ arise in this manner. Then the action of $\varphi$ on $\mathcal{A}$ preserves cyclic order.

Finally, let $\mathcal{A}$ be a pairwise disjoint collection of closed, connected sets in the punctured plane $\mathbb{C}^{*}=\mathbb{C} \backslash\{0\}$, and that the closure of each element of $\mathcal{A}$ contains both 0 and $\infty$. Then we can define the cyclic order at $\infty$ on $\mathcal{A}$, by replacing each element of $\mathcal{A}$ by an unbounded connected subset that is closed in $\mathbb{C}$, and applying the above definition. Analogously, we can define a cyclic order on $\mathcal{A}$ at 0 . It is easy 
to see (again using the plane separation theorem) that both orders coincide, and depend only on $\mathcal{A}$ rather than any choices made in the construction.

REMARK There are some subtleties to the definition of circular order on connected sets, compared with the case of arcs to infinity which has been previously considered in the complex dynamics literature. For example, note that the assumption that the sets in $\mathcal{A}$ are closed is crucial. Indeed, consider the case of a Knaster buckethandle continuum $X$, whose terminal point (that is, the initial point of the half-ray running through all of the endpoints of the complementary intervals of the ternary Cantor set) has been placed at $\infty$, and consider the collection of path-connected components of this set. Every such component is unbounded and connected, but since each component accumulates everywhere upon $X$, there is no sensible circular order among them.

\section{Appendix: Unbounded Postsingular Sets}

As mentioned in the introduction, the Douady-Hubbard landing theorem no longer holds for polynomials with escaping singular values. It is still true that every repelling (or parabolic) periodic point is accessible from the basin of infinity, and even by a dynamic ray, if we extend this notion appropriately to the case where the ray passes through critical points; compare [EL89, LP96]). However, it is possible for the set of landing rays to be uncountable, and for none of these rays to be periodic; compare [GM93, Appendix C] and [LP96].

Let us now briefly discuss the case of transcendental entire functions $f$ with unbounded postsingular set $\mathcal{P}(f)$. When $f \notin \mathcal{B}$, the structure of the escaping set may change dramatically within a given parameter space (compare [RS17, Appendix B]), and hence it is not clear whether questions concerning the landing of rays or dreadlocks are even meaningful in this setting. Let us hence restrict to the case of $f \in \mathcal{B}$.

First suppose that $f$ has an escaping singular value. In addition to the abovementioned behaviour that occurs already for polynomials, it is also possible for a repelling periodic point to not be accessible from the escaping set at all (by hairs or dreadlocks). Indeed, this is the case for the fixed point of the exponential map $z \mapsto e^{z}$ having imaginary part between 0 and $\pi$, and shows that the question of landing behaviour at periodic points becomes considerably more subtle when $\mathcal{P}(f)$ is unbounded.

However, consider now the full family of exponential maps, $f_{a}: z \mapsto e^{z}+a$. Suppose that the singular value $a$ has an unbounded orbit but does not belong to the escaping set. Then $f_{a}$ is criniferous. In [Rem06a], it is shown that that all periodic hairs of $f_{a}$ land. Conversely, every periodic point, with the exception of at most one periodic orbit, is the landing point of a periodic hair. The exceptional orbit cannot be parabolic, but it is an open question whether it can be repelling. It is shown in [Rem06a] that a plausible conjecture about parameter space of exponential maps (the "no ghost limbs conjecture") would imply that this is not the case. 
Hence it is plausible that the Douady-Hubbard landing theorem remains valid for exponential maps as above, which raises the question whether the main theorem of our paper may also have an extension for functions $f \in \mathcal{B}$ with unbounded but non-escaping singular orbits. A crucial step is to ensure the landing of periodic rays (or dreadlocks). Indeed, if periodic rays land and the function has good geometry in the sense of [RRRS11], one can show that the number of rationally invisible repelling periodic orbits is bounded by the number of free singular values [BF20], just as for the exponential family. Unfortunately, the proofs in [Rem06a] that periodic rays land use sophisticated results on the structure of the (one-dimensional) parameter space of exponential maps, and it appears that fundamentally new approaches would be required to resolve this question in full generality.

\section{Acknowledgments}

We are extremely grateful to Dave Sixsmith and to David Pfrang for their many and extraordinarily helpful suggestions that considerably improved the presentation of the paper. We also thank Daniel Meyer for interesting comments, particularly a suggestion on the presentation of cyclic order in Section 13.

Open Access This article is licensed under a Creative Commons Attribution 4.0 International License, which permits use, sharing, adaptation, distribution and reproduction in any medium or format, as long as you give appropriate credit to the original author(s) and the source, provide a link to the Creative Commons licence, and indicate if changes were made. The images or other third party material in this article are included in the article's Creative Commons licence, unless indicated otherwise in a credit line to the material. If material is not included in the article's Creative Commons licence and your intended use is not permitted by statutory regulation or exceeds the permitted use, you will need to obtain permission directly from the copyright holder. To view a copy of this licence, visit http://creativecommons.org/licenses/by/4.0/.

Publisher's Note Springer Nature remains neutral with regard to jurisdictional claims in published maps and institutional affiliations.

\section{References}

[ABR] Simon Albrecht, Anna M. Benini, and Lasse Rempe. Conjugacy classes of finite-order transcendental entire functions, In: preparation.

[ALS11] Artur Avila, Mikhail Lyubich, and Weixiao Shen. Parapuzzle of the Multibrot set and typical dynamics of unimodal maps. J. Eur. Math. Soc. (JEMS), (1)13 (2011), 27-56.

[AR17] Nada Alhabib and Lasse Rempe-Gillen. Escaping endpoints explode. Comput. Methods Funct. Theory, (1)17 (2017), 65-100.

[Bak84] Irvin Noel BAKER. Wandering domains in the iteration of entire functions. Proc. London Math. Soc. (3), (3)49 (1984), 563-576. 
[BDH+99] Clara Bodelón, Robert L. Devaney, Michael Hayes, Gareth Roberts, Lisa R. Goldberg, and John H. Hubbard. Hairs for the complex exponential family. Internat. J. Bifur. Chaos Appl. Sci. Engrg., (8)9 (1999), 1517-1534.

[Ben11] Anna Miriam Benini. Triviality of fibers for misiurewicz parameters in the exponential family. Conform. Geom. Dyn. (9)15 (2011), 133-151.

[Ben15] Anna Miriam Benini. Expansivity properties and rigidity for non-recurrent exponential maps. Nonlinearity (7)28 (2015), 2003-2025.

[Ben16] Anna Miriam Benini. A note on repelling periodic points for meromorphic functions with a bounded set of singular values. Rev. Mat. Iberoam. (1)32 (2016), $267-274$.

[BF15] Anna Miriam Benini and Núria FAgella. A separation theorem for entire transcendental maps. Proc. Lond. Math. Soc. (3) (2)110 (2015), 291-324.

[BF17] Anna Miriam Benini and Núria FAGELLA. Singular values and non-repelling cycles for entire transcendental maps, 2017, to appear in Indiana Univ. Math. $\mathrm{J}$.

[BF20] Anna Miriam Benini and Núria Fagella. A bound on the number of rationally invisible repelling orbits. Advances in Mathematics 370, (2020).

[Bin51] R. H. Bing. Snake-like continua. Duke Math. J. 18 (1951), 653-663.

[BK07] Krzysztof BARAŃsKi and Bogusława KarPińskA. Coding trees and boundaries of attracting basins for some entire maps. Nonlinearity (2) 20 (2007), 391-415.

[BL02] A. BlOKH and G. Levin. An inequality for laminations, Julia sets and "growing trees". Ergodic Theory Dynam. Systems (1)22 (2002), 63-97.

[BL14] Anna Miriam Benini and Mikhail Lyubich. Repelling periodic points and landing of rays for post-singularly bounded exponential maps. Annales de l'Institut Fourier (4)64 (2014), 1493-1520.

[Čec69] Eduard ČEch, Point sets, translated from the Czech by Aleš Pultr, Academic Press, New York-London, 1969.

[Den14] Aslı Deniz. A landing theorem for periodic dynamic rays for transcendental entire maps with bounded post-singular set. J. Difference Equ. Appl. (12)20 (2014), 1627-1640.

[Dev84] Robert L. Devaney. Julia sets and bifurcation diagrams for exponential maps. Bull. Amer. Math. Soc. (N.S.) (1)11 (1984), 167-171.

[DGH86] Robert L. Devaney, Lisa R. Goldberg, and John H. Hubbard. A dynamical approximation to the exponential map by polynomials, Preprint, 1986.

[DH85] Adrien DouAdy and John HubBard. Etude dynamique des polynômes complexes, Prépublications mathémathiques d'Orsay (1984 / 1985), no. 2/4.

[DJ02] Robert L. Devaney and Xavier Jarque. Indecomposable continua in exponential dynamics. Conform. Geom. Dyn. 6 (2002), 1-12.

[DJR05] Robert L. Devaney, Xavier Jarque, and Mónica Moreno Rocha. Indecomposable continua and Misiurewicz points in exponential dynamics. Internat. J. Bifur. Chaos Appl. Sci. Engrg. (10)15 (2005), 3281-3293.

[DK84] Robert L. Devaney and Michał KRYCH. Dynamics of exp(z). Ergodic Theory Dynam. Systems (1)4 (1984), 35-52.

[DT86] Robert L. Devaney and Folkert Tangerman. Dynamics of entire functions near the essential singularity. Ergodic Theory Dynam. Systems (4)6 (1986), 489-503.

[EFJS19] Vasiliki Evdoridou, Núria Fagella, Xavier Jarque, and David J. Sixsmith. Singularities of inner functions associated with hyperbolic maps. J. Math. Anal. Appl. (1)477 (2019), 536-550. 
[EL89] Alexandre È. Eremenko and Genadi M. Levin. Periodic points of polynomials. Ukrain. Mat. Zh. (11)41 (1989), 1467-1471, 1581.

[EL92] Alexandre È. Eremenko and Mikhail Yu. Lyubich. Dynamical properties of some classes of entire functions. Ann. Inst. Fourier (Grenoble) (4)42 (1992), 989-1020.

[Fat26] Pierre Fatou. Sur l'itération des fonctions transcendantes entières. Acta Math. 47 (1926), 337-370.

[GM93] Lisa R. Goldberg and John Milnor. Fixed points of polynomial maps. II. Fixed point portraits. Ann. Sci. École Norm. Sup. (4) (1)26 (1993), 51-98.

[Hub93] John Hamal HubBARD. Local connectivity of Julia sets and bifurcation loci: three theorems of J.-C. Yoccoz, Topological methods in modern mathematics (Stony Brook, NY, 1991), Publish or Perish, Houston, TX, 1993, pp. 467-511.

[JT02] Gerald JungCK and Mathew Timm. Another characterization of non-separating planar continua, Topology Proc. 26 (2001/02), no. 1, 235-246, (Proceedings of the Spring Topology and Dynamical Systems Conference, 2001).

[Kiw02] Jan KIwI. Wandering orbit portraits. Trans. Amer. Math. Soc. 354 (2002), 1473-1485.

[LP96] Genadi Levin and Feliks PRZYTYCKI. External rays to periodic points. Israel Journal of Mathematics (1)94 (1996), 29-57.

[Lyu83] Mikhail LyUBiCH. Entropy properties of rational endomorphisms of the riemann sphere. Ergodic Theory Dynam. Systems (3)3 (1983), 351-385.

[Mań12] Roman MAŃKA. Results and problems in fixed point theory for tree-like continua. Topology Proc. 39 (2012), 113-130.

[Mih10] Helena Minaljević-Brandt. A landing theorem for dynamic rays of geometrically finite entire functions, 2010, pp. 696-714.

[Mil06] John Milnor. Dynamics in one complex variable, third edition ed., vol. 160, Annals of Mathematics Studies, Princeton University Press, Princeton, NJ, 2006.

[Min17] David Minda. Quotients of hyperbolic metrics. Comput. Methods Funct. Theory (4) 17 (2017), 579-590.

[MR13] Helena Mihaljević-Brandt and Lasse Rempe-Gillen. Absence of wandering domains for some real entire functions with bounded singular sets. Math. Ann. (4)357 (2013), 1577-1604.

[Nad92] Sam B. Nadler, Jr., Continuum theory. An introduction, Monographs and Textbooks in Pure and Applied Mathematics, vol. 158, Marcel Dekker Inc., New York, 1992.

[Pfr19] David Pfrang. Homotopy Hubbard trees for post-singularly finite transcendental entire functions, doctoral thesis, Jacobs University Bremen, 2019.

[Pom92] Christian Pommerenke, Boundary behaviour of conformal maps, Grundlehren der Mathematischen Wissenschaften, vol. 299, Springer-Verlag, Berlin, 1992.

[PRS18] David Pfrang, Michael Rothgang, and Dierk Schleicher. Homotopy hubbard trees for post-singularly finite exponential maps, 2018.

[Prz94] Feliks Przytycki. Accessibility of typical points for invariant measures of positive Lyapunov exponents for iterations of holomorphic maps. Fund. Math. (3)144 (1994), 259-278.

[Rem06a] Lasse REMPE. A landing theorem for periodic rays of exponential maps. Proc. Amer. Math. Soc (9)134 (2006), 2639-2648.

[Rem06b] Lasse REMPE. Topological dynamics of exponential maps on their escaping sets. Ergodic Theory Dynam. Systems (6)26 (2006), 1939-1975. 
[Rem07a] Lasse REMPE. On a question of Eremenko concerning escaping sets of entire functions. Bull. London Math. Soc. 39 (2007), no. 4, 661-666.

[Rem07b] Lasse Rempe. On nonlanding dynamic rays of exponential maps. Ann. Acad. Sci. Fenn. Math. (2)32 (2007), 353-369.

[Rem08] Lasse Rempe. Siegel disks and periodic rays of entire functions. J. Reine Angew. Math. 624 (2008), 81-102.

[Rem09] Lasse REMPE. Rigidity of escaping dynamics for transcendental entire functions. Acta Math. (2)203 (2009), 235-267.

[Rem16] Lasse REMPE. Arc-like continua, Julia sets of entire functions, and Eremenko's Conjecture, Preprint, 2016, arXiv:1610.06278.

[Roe08] Pascale Roesch. On local connectivity for the Julia set of rational maps: Newton's famous example. Ann. of Math. (2) (1)168 (2008), 127-174.

[Rot05] Günter Rottenfusser. Dynamical Fine Structure of entire transcendental functions, doctoral thesis, International University Bremen, 2005.

[RRRS11] Günter Rottenfusser, Johannes Rückert, Lasse Rempe, and Dierk SchleICHER. Dynamic rays of bounded-type entire functions. Ann. of Math. (2) (1)173 (2011), 77-125.

[RRS10] Lasse Rempe, Philip J. Rippon, and Gwyneth M. Stallard. Are Devaney hairs fast escaping?. J. Difference Equ. Appl. (5-6)16 (2010), 739-762.

[RS08a] Lasse REMPE and Dierk SchleICHER. Combinatorics of bifurcations in exponential parameter space, Transcendental Dynamics and Complex Analysis (P. Rippon and G. Stallard, eds.), London Math. Soc. Lecture Note Ser., vol. 348, Cambridge Univ. Press, 2008, pp. 317-370.

[RS08b] Günter RotTenfusser and Dierk Schleicher. Escaping points of the cosine family, Transcendental dynamics and complex analysis, London Math. Soc. Lecture Note Ser., vol. 348, Cambridge Univ. Press, Cambridge, 2008, pp. 396-424.

[RS17] Lasse Rempe-Gillen and Dave Sixsmith. Hyperbolic entire functions and the Eremenko-Lyubich class: class $\mathcal{B}$ or not class $\mathcal{B}$ ?. Math. Z. (3-4)286 (2017), 783-800.

[RvS11] Lasse Rempe and Sebastian van Strien. Absence of line fields and Mañé's theorem for nonrecurrent transcendental functions. Trans. Amer. Math. Soc. (1)363 (2011), 203-228.

[RY08] Pascale RoEsch and Yongcheng YIN. The boundary of bounded polynomial Fatou components. C. R. Math. Acad. Sci. Paris (15-16)346 (2008), 877-880.

[SZ03a] Dierk SchleICHER and Johannes Zimmer. Escaping points of exponential maps. J. London Math. Soc. (2) (2)67 (2003), 380-400.

[SZ03b] Dierk Schleicher and Johannes Zimmer. Periodic points and dynamic rays of exponential maps. Ann. Acad. Sci. Fenn. Math. 28 (2003), 327-354.

[Why42] Gordon Thomas WhyBurn. Analytic Topology, American Mathematical Society Colloquium Publications, v. 28, American Mathematical Society, New York, 1942.

[Wor18] Stephen WorsLey. The topology of postsingularly finite exponential maps, doctoral thesis, University of Liverpool, 2018. 
A. M. Benini

Dipartimento di Scienze Matematiche Fisiche e Informatiche, Università di Parma, Parma, Italy.

ambenini@gmail.com

L. REMPE

Department of Mathematical Sciences, University of Liverpool, Liverpool L69 7ZL, UK.

1.rempe@liverpool.ac.uk

Received: January 2, 2020

Accepted: July 7, 2020 